\title{
Developing Learning Progressions in Support of the New Science Standards: A RAPID Workshop Series
}

Aaron Rogat

Follow this and additional works at: https://repository.upenn.edu/cpre_researchreports

Part of the Curriculum and Instruction Commons, Educational Assessment, Evaluation, and Research Commons, and the Science and Mathematics Education Commons

\section{Recommended Citation}

Rogat, Aaron. (2011). Developing Learning Progressions in Support of the New Science Standards: A RAPID Workshop Series. CPRE Research Reports.

Retrieved from https://repository.upenn.edu/cpre_researchreports/66

View on the CPRE website.

This paper is posted at ScholarlyCommons. https://repository.upenn.edu/cpre_researchreports/66

For more information, please contact repository@pobox.upenn.edu. 


\title{
Developing Learning Progressions in Support of the New Science Standards: A RAPID Workshop Series
}

\author{
Abstract \\ The hypothetical learning progressions presented here are the products of the deliberations of two \\ working groups of science education researchers, each group also including a state science curriculum \\ supervisor, organized by the Consortium for Policy Research in Education (CPRE), with support from the \\ National Science Foundation. Their charge was to produce hypothetical learning progressions describing \\ the pathways students might be expected to follow as they acquire deep understanding of two of the core \\ learning goals set by the National Research Council's (NRC) Committee on a Conceptual Framework for \\ the New K-12 Science Education Standards. The goals in question address students' understanding of the \\ structure, properties, and transformations of matter in the physical sciences and of the flow of matter and \\ energy in ecosystems in the life sciences. These two core goals were chosen because a good bit of \\ research has been done on children's learning in these areas, some of it carried out by members of our \\ working groups. These hypothetical learning progressions are intended to inform those who are working \\ on the new national science standards, to serve as tools for those charged with developing curriculum \\ and assessments to implement the new standards, and to encourage others to undertake the theoretical \\ and empirical work needed to fill important gaps in our knowledge about learning progressions. \\ Disciplines \\ Curriculum and Instruction | Educational Assessment, Evaluation, and Research | Science and \\ Mathematics Education

\section{Comments} \\ View on the CPRE website.
}




\title{
Developing Learning Progressions in Support of the New Science Standards: A RAPID Workshop Series
}

\author{
Prepared by \\ Aaron Rogat, Principal Investigator \\ With \\ Charles (Andy) Anderson, Jacob Foster, Fred Goldberg, Jennifer Hicks, David \\ Kanter, Joseph Krajcik, Richard Lehrer, Brian Reiser, and Marianne Wiser
}

November 2011

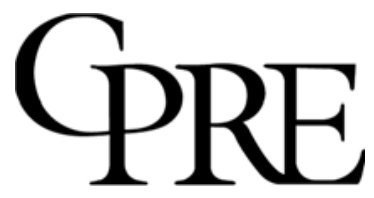

Consortium for Policy Research in Education

Teachers College, Columbia University 
The research presented in this report was funded by a National Science Foundation (NSF) grant (DRL-1051144) to the Consortium for Policy Research in Education (CPRE). Opinions expressed in this report are those of the authors and do not necessarily reflect the views of the NSF, CPRE, or its institutional members. 


\section{Preface}

The hypothetical learning progressions presented here are the products of the deliberations of two working groups of science education researchers, each group also including a state science curriculum supervisor, organized by the Consortium for Policy Research in Education (CPRE), with support from the National Science Foundation. Their charge was to produce hypothetical learning progressions describing the pathways students might be expected to follow as they acquire deep understanding of two of the core learning goals set by the National Research Council's (NRC) Committee on a Conceptual Framework for the New K-12 Science Education Standards. The goals in question address students' understanding of the structure, properties, and transformations of matter in the physical sciences and of the flow of matter and energy in ecosystems in the life sciences. These two core goals were chosen because a good bit of research has been done on children's learning in these areas, some of it carried out by members of our working groups. These hypothetical learning progressions are intended to inform those who are working on the new national science standards, to serve as tools for those charged with developing curriculum and assessments to implement the new standards, and to encourage others to undertake the theoretical and empirical work needed to fill important gaps in our knowledge about learning progressions.

At the end of July 2011 the NRC's Framework Committee released its report, A Framework for K-12 Science Education: Practices, crosscutting concepts, and core ideas (NRC, 2012). The Framework was the first step in a two-step process to produce a new set of science standards for voluntary adoption by states. The second step-the development of a set of standards based on this framework-is being led by Achieve Inc., working in collaboration with the states and offering opportunities for input from science educators and the public. Accordingly, the Framework is a parsimonious description of what every student should know and understand by the end of high school in order to be scientifically literate, able to function as a responsible citizen and participant in the modern economy, and able to pursue further learning as his or her career and life interests might require.

The Framework identifies core ideas in four key disciplinary areas - physical sciences, life sciences, earth and space sciences, and engineering, technology, and the application of science-along with cross-cutting concepts that unify the study of science and engineering through their common application across fields and the scientific and 
engineering "practices" that are central to the ways in which knowledge in these fields is produced, tested, and used. However, the committee's report makes it clear that they consider the evidence base for their recommendations-particularly those that concern how students learn the core ideas and practices of science and how to support that learning-to be incomplete. In the final chapter they outline an agenda for research and development that would over time result in a firmer basis for setting standards and designing supportive curricula, assessments, and professional development.

Among the topics the committee identifies as key foci for further research are:

- Studies of the typical trajectories of student learning to identify preconceptions of a topic and the expected progression of student understanding;

- Identification of the educational experiences required to move students along the expected learning path;

- Specification of the general and discipline-specific norms and practices that define the expectations for students' and teachers' interactions in the classroom;

- Studies of how young people of different backgrounds, cultures, races, genders, abilities, and languages engage in learning and can become full participants in the scientific classroom community;

- Research on methods of assessing student understanding of key concepts in science.

The committee suggests that:

"an especially important line of inquiry should involve learning progressions that embed the core ideas and practices spelled out in this document [the Framework]. Such research may focus on a particular core idea and ask what sequence of learning experiences, including engagement in practices, around that idea best advance student understanding and address, or take advantage of, common naive conceptions. It is worth noting that, because R\&D on learning progressions in science is at an early stage, many aspects of the core ideas and their progressions over time with instruction (as sketched out in the framework) remain unexplored territory."

\section{Moving into Unexplored Territory}


The CPRE project reported on here represents one possible approach to the development of learning progressions. It should be seen as a supplement to the work that Achieve is doing in developing a more complete set of standards based on the NRC Framework. We assume that those standards will take the form of more detailed propositional descriptions of the knowledge and skills students should master by the end of each grade band, along with specifications for the kinds of performances that would demonstrate their mastery.

Our project focused on two of the core concepts in the NRC Framework, one in the physical sciences-understanding the structure, properties, and transformations of matter-and one in the life sciences-understanding the flow of matter and energy in living systems. These two concepts are central to a modern understanding of the physical and living worlds, and taken together they illustrate the way in which a fundamental understanding of the physical world supports a deeper understanding of how living systems work. The purpose of our project has been to provide carefully worked-out examples of how to approach the revision of science education standards, curriculum, and assessment from the perspective of teaching and learning progressions and what the benefits of this approach might be.

The progressions perspective is rooted in the observation that all human learners are actively trying to make sense of their world. Children and students bring their own prior conceptions, often naïve to be sure, but also compelling and persistent, to the learning of any subject. To be effective, instruction has to take those conceptions into account and build on them, as well as modify them through instruction, if students are to develop the more sophisticated (and more general, flexible, and powerful) conceptual structures that are the goals of science education. The progressions hypothesis is that learning any complex subject is not simply a matter of acquiring sets of facts and propositions but rather that learners actively try to organize knowledge in ways that make sense, that teachers should try to help them do that in ways that are consistent with the goals of instruction, and that it should be possible to describe student learning in terms of an ordered succession of conceptual structures or "levels" that become increasingly sophisticated and powerful over time.

The distinctive feature of a progressions approach is that it pays careful attention to the nature of students' conceptual understanding and whether and how it is changing, but it by no means assumes that these changes will take place at all, or that they will develop 
as desired, without specific and responsive instruction, which is why we stress the idea of "teaching and learning progressions" rather than just "learning progressions." The progressions hypothesis would suggest that some sets and sequences of these conceptual levels are more likely than others, particularly in the early and more naïve stages, and that instructional experiences designed to foster those sequences may have a better chance of leading to the desired goals, but it does not hold that there is any one likely, or ideal, sequence. Identifying the sequences and determining how to foster them most effectively is a matter of an iterative design, testing, and re-design process. As the NRC report suggests, there are some areas of science in which initial empirical work of this sort has already been done (see Corcoran, Mosher, \& Rogat, 2009 for examples), including the domains we chose for the focus of our project matter and the flow of matter and energy. CPRE recruited some of the key science educators and learning scientists who have done this work to participate in two working groups charged with developing more elaborate "hypothetical learning progressions" for describing how students might acquire more sophisticated understanding of these two core ideas. Each working group was joined by a state science supervisor because we wanted the work to be disciplined by a clear sense of the policy and practice contexts in which we expected it to be used. The specific composition of the working groups can be found in Appendix A.

We use the term "hypothetical learning progressions" (hereafter HLP) to emphasize that, while many of the participants have concrete experience with students' learning in these domains, they have not done the additional empirical work necessary to develop detailed descriptions of how students' understanding might develop over time with adequate instruction. Rather, these HLP'S are good faith hypotheses based on the best currently available evidence, and extrapolations from it, about how students' understanding in these areas develops. Specifically, the HLP's define the typical levels that students' understanding might be expected to go through given instructional exposure to the phenomena that the scientific concepts they are learning have been developed to explain, and given guided experience in trying to reason and argue about those phenomena in the ways that working scientists might do. As we will describe in more detail below, our central finding is that in both domains the most compelling way to characterize the successive levels of understandings we think students will proceed through if they are to reach the goals for high school science, is to frame them as a series of successively more sophisticated explanatory models, that take into account more and more of the relevant phenomena and that move from naïve explanations based on folk concepts and directly observable interactions to models that deal with 
hypothesized interactions among constructs and entities that are observable or measured only with sophisticated tools and/or inferred from their observed effects.

Given that the central goal of science is understanding and explanation, it probably is not surprising that students' learning of science can be effectively described in terms of progressively more sophisticated explanatory models. Being able to characterize in some detail the ways in which these models are appreciated by students, and how they change with specific experiences and guided practice in thinking scientifically, should provide useful insights into how to design standards, curricula, and assessments that help all involved - educators and students in particular - to be more sensitive to where students are coming from and where they are going at all points along their paths in school, as well as where they are at any particular time and what might be helpful in moving them ahead.

Developers of LPs often begin their work by proposing a hypothetical LP that is based on a review of existing knowledge about how students learn a particular concept or topic, as well as the naïve conceptions students may hold about that concept and the common errors or problems they may encounter in coming to understand and apply it. They often enlist the help of scientists within the domain to help them identify key concepts and principles that are relevant to the core idea and to organize them into a coherent and accurate framework. The NRC framework, has essentially accomplished this task. The next step in the development of an HLP is for education researchers to further review existing research on how students learn the core concept in question. This research is used to refine the coherence of the framework and describe a sequence of the ways in which students' ideas are likely to change and develop over time as (and if) they move toward a conceptualization of the core concept that approximates the conception held by scientists. Importantly, the researchers constructing an initial HLP must identify the prior ideas students hold as they begin to explore and study the natural phenomena explained by the core concept as well as the key intermediate ideas that students might take advantage of as they develop more sophisticated knowledge and skill. The HLP is subsequently tested in practice and revised based on assessment evidence. Such assessments may be a combination of paper and pencil items or clinical interviews or teacher or researcher evaluations of students' work, but they must be able to probe what students understand and can do with their knowledge of the targeted concepts and practices. Most developers of HLPS also operationally define what student understanding is likely to look like at each level by identifying learning performances that specify how students, if they were at a given 
level of the progression, would use their knowledge at that level to explain, model, or predict natural phenomena relevant to the core idea. A good HLP has gone through multiple rounds of such iterative testing and revision based on the assessment results; this process builds confidence that an HLP is reasonably valid and can characterize the progression for most students (Corcoran et al., 2009).

Thus, the end products of an effort to develop a useful HLP should include: a) a framework, informed by research, that describes how students' ideas can change and develop across at least two grade bands; and b) learning performances that can inform the development of assessments that measure change in student understanding and can place a student along the trajectory towards the instructional goal. A useful HLP ought also to specify the associated instructional experiences that are hypothesized to promote students' progress and that anticipate the content of the learning performances.

\section{What has this project produced?}

As we already have noted, the project's two working groups undertook development of HLPs for two of the core ideas in the physical and life sciences sections of the NRC's Framework for K-12 science education. The two HLPs cover students' development of core concepts concerning: 1) the structure, properties and transformation of matter, in the physical sciences, and 2) the flow of matter and energy in ecosystems, in the life sciences.

Two representations for each HLP are presented in this report: a narrative and a table. Below we describe the key parts of these representations and what they may be used for.

The table representations. The table representations of the HLPS are the core products of our work. The tables represent the working groups' best judgments of how to describe the significant steps or levels of understanding that students are likely to go through if they progress from the naïve explanations of physical and biological phenomena that they come to school with, to acquiring and using reasonable approximations of contemporary scientific explanatory models as described by the NRC framework. These are based on the experience of the members of the working groups, on reasoning from the logic of the scientific disciplines, and on research evidence of the ways students' understanding and explanations of the core science concepts and 
related phenomena in fact develop in current school settings, both conventional and experimental.

The children's explanations described in the early levels of the two HLPs are not of course scientifically adequate explanations of the relevant phenomena, but they do represent relatively coherent views that act as useful scaffolds to focus students' attention on aspects of the phenomena upon which they can build their thinking as they move to the next level, closer to the canonical contemporary view. We try to identify useful ideas, concepts, and experiences that can help to focus students' attention on important aspects of phenomena and facilitate their appreciation of observations, instrumentation, contrasts, and other evidence that support their eventual adoption of more sophisticated views and concepts (Wiser \& Smith, In Press). Where possible we point out likely misconceptions or conceptual dead ends that could make moving to the next level of understanding difficult or produce partial or confused consolidations in student thinking. The field has more evidence of these useful ideas and impediments to instruction and student thinking at the earlier levels, and so as we move to the higher levels of the two HLPs our descriptions are rooted less in direct evidence of students' thinking and come more to resemble textbook or standards-like descriptions of disciplinary models and findings. In that sense they become even more "hypothetical" representing reasonable first approximations of how students might think at the more advanced levels and providing guidance for focusing instruction that could test these hypotheses in practice and produce evidence for revising them based on more direct measures of how students' thinking actually progresses..

One of the main approaches to developing learning progressions has focused on design of assessments that could reveal how students are thinking about and explaining natural phenomena, and whether and how those explanations are becoming more sophisticated over the grades. Hypotheses are then formed about the nature and order of the levels of student knowledge and skill, and then additional cohorts of students are tested (often on a cross-sectional basis, but sometimes longitudinally) to see whether the hypothesized order and structure holds up in the new assessment results. This approach has given rise to an emphasis in the field of learning progressions research on the concept of "learning performances" - descriptions of what students' who were at a defined level on a progressions would be able to do on appropriately designed assessment items or tasks (and that students at a "lower" level would not be able to do, or would do differently) (refs. to Mark Wilson; Mohan, Anderson, et al; Corcoran et. al., 2009 the pages with the extended definition of a progression that we used in the Brief). 
Given their grounding in assessment design, learning performances can often be interpreted as things that students should be able to do. This is particularly true when they are discussed in the context of standards, but it is important to keep in mind that our HLPs are really teaching and learning progressions - students are not expected to progress very far without instruction, and it is not likely that they will be capable of the learning performances expected at a given level of a progression unless they have had extensive experience during instruction with carrying out the same kinds of performance. Therefore the learning performances presented in our tables represent what students would be likely to be able to do at a given level with adequate instruction and are often framed in the language of assessment, particularly as they use the label "criteria" to discuss the expected attributes of students' performances. We need to stress in advance that all of those performances also could, and should, be read as descriptions of suggested learning experiences students ought to be exposed to during instruction. We use the label "learning experiences and performances" for sections that describe the levels in the tables, but we have not always been able to rephrase them so that the instructional intent is as clear as the assessment orientation.

A further caution involves our framing of the performances in terms of the science "practices" that are involved along with core scientific knowledge in what students are asked or expected to be able to do. In a standards context, the question of how well students are expected to perform with the required content at each grade or grade band always arises. Close consideration of performance standards makes it clear that answering the how well question usually involves being able to say something about the quality of students' reasoning, use of evidence, argument, or understanding of scientific methods. In other words, it has to do with where they stand in some progression of their abilities to handle scientific practices as they use or apply their scientific knowledge. Our working groups identified three "clusters" or sub-sets of related science practices from the seven practices listed in the NRC framework and tried to provide examples of how well students who had reached a particular level in a progression would be likely to be able to demonstrate the use of practices from each of the three clusters - 1.Explaining, predicting, developing explanatory models; 2.Designing investigations, collecting, representing, and analyzing data; and 3. Constructing scientific arguments - as they apply them to the phenomena they are able to attend to and explain at that level. See Appendix B for a fuller description of these clusters. Again, as framed here, these sound very much like performance assessment items, but they also should be read as encompassing the kinds of learning experiences students 
should be having during instruction as they experiment, explain, and argue about their experiments and explanations. We do not have research evidence at this point to justify these particular hypotheses about how practices and content will work together at each level, though the members of our groups do have a good deal of practical experience with students learning across these grade levels, so there is some grounding for these examples. They should be interpreted as an initial attempt to provide guidance for designing both instruction and assessments that would encourage students' progress along the lines drawn by the HLPs.

Finally, the working groups have associated the levels of the HLPs with particular grade bands. These associations are derived in part from where the related content knowledge has been placed in the NRC framework, but we would stress that our real emphasis is on the order of the levels rather than on their particular grade placements. The grade placements do have some grounding in our experience with typical students' understanding, particularly at the earlier ages, but some children will reach these levels considerably earlier, substantial numbers may be later, and many currently do not reach the higher levels at all during their school careers.

The Tables: There are two HLP tables - one on the structure and properties of and transformations of matter, and one on the flow of matter and energy in ecosystems. Each level has a label that attempts to capture in a few words the character of the students' explanation or explanatory model at that level. The grade band thought to be associated with the level is given, but as we say above, we consider the order of the levels to be more significant than the particular grade placement.

Overview: The overview provides a thumbnail sketch of the character of the explanation or explanatory model that students at the level would characteristically offer for the relevant phenomena.

Students' explanation or explanatory model: This is a more detailed summary of the conceptual knowledge that students can be expected to apply to explain and think about relevant phenomena at a particular level. Since we aimed to fully explicate the explanations or models that students at a particular level would develop, the ideas may not fall neatly into one core idea from the NRC Framework. We attempt, particularly at the earlier levels, to describe this using language and terms that students would use (noting the relationships to and differences from more scientific terminology as needed). It is framed as students' answers to "focal questions" about the natural phenomena that are the focus of the HLP. At some of the levels in the HLPs, in order to maintain the 
integrity of the students' actual conceptual structures, when we have evidence about that or strong bases for expecting them to make broader connections, we have drawn on core ideas in the NRC framework other than those set out in the sections we are specifically tracking. Aside from minor differences in emphasis and organization, however, we do not think we have gone beyond the core ideas found in the NRC framework as a whole.

What has changed in students' thinking from the previous level: This section both elaborates further on students' thinking at each level and contrasts it with the previous level, noting changes in the ways particular phenomena are explained and noting new phenomena that are now considered and encompassed and new ways of reasoning and arguing about phenomena and evidence.

Student ideas and boundaries: This covers the useful or productive concepts and experiences that reorient students' attention within the level and/or facilitate moving to the next level. It again draws some contrasts with prior, more naive ideas, and identifies concepts that may present particular difficulties and others that students may hold that will get in the way of their progress. "Boundaries" represent suggestions about concepts and issues that are not necessary, or maybe inappropriate to expect a student to learn at this level.

Rationales and notes: This provides an area for additional thoughts and observations about students' thinking, explanations, and associated experiences that have not yet been fully incorporated in the preceding sections.

Notes on how students demonstrate and apply their knowledge: These are further notes that suggest topics and phenomena that students might reason about and apply their explanations to. These notes also summarize the themes and changes in students' explanations, investigations, or arguments within and across adjacent levels. They are to be used as a basis for designing learning experience and learning performances of the sort described in the next section.

Sample learning experiences and learning performances: As described above these are suggestions for possible ways students at a given level might respond to requests to explain, argue, reason about, and gather evidence about relevant natural phenomena. They also describe what students might be asked to do during instruction as they progress toward the more sophisticated explanations at the next level, although 
we note that the instructional experiences identified in the progressions are not necessarily sufficient to promote the shifts in thinking expected from one level to the next. The experiences and performances combine scientific practices with the learning of scientific concepts and content.

The Narratives. The narrative for each HLP is meant to provide a big picture overview of the progression and how it was developed. It addresses which core ideas from the NRC framework are addressed in this learning progression. Additionally, it summarizes three aspects of the progression: 1) the key features of students' explanations or models, and changes in those explanations from level to level, 2) useful or productive ideas that may serve as leverage from one level of understanding to another, and 3) trends and changes in learning experiences and performances across the levels. There is also a simplified outline of each HLP that addresses each of these key features in brief form. Given that the table representation is fairly dense and does not allow for easy comparison of all the levels of the progression at the same time, the narrative may facilitate reading and interpreting the associated table. It may be useful to read the narrative before reading the table for each HLP.

\section{Concluding Remarks}

Our intention is that these draft HLPs should prove to be useful to state and district science education supervisors and others involved in developing new standards, curriculum, and assessments. We hope they will have direct relevance for thinking and making decisions about the following issues:

What aspects of content should standards focus on in order to develop a coherent vertical sequence of ideas that would support students in reaching the upper levels of understanding targeted in science standards?

In particular, what curriculum content and forms of instruction would support students in the development of the levels of knowledge and skill targeted in the upper levels of compulsory science education?

With respect to assessment selection issues: How can we describe where students are in their progress towards the learning goals targeted by standards, and how can we choose or design assessments that can report their progress in those terms? How do we ensure good alignment between assessment for summative and formative purposes so that it supports instruction and informs policy appropriately? 
We offer these draft HLPs as concrete examples of what work carried out from a learning progressions perspective has to offer through synthesis and extrapolation of research on how students' learning develops over time with respect to two core areas of science knowledge. We hope this will give enough of an idea of how this approach might be useful, so that it will help to justify carrying out comparable and more extensive efforts for the rest of the core ideas and goals of science instruction. 


\section{References}

Corcoran, T., Mosher, F., \& Rogat, A. (2009). Learning progressions in science: An evidence-based approach to reform (No. RR-63). Philadelphia: Consortium for Policy Research in Education, University of Pennsylvania.

Hess, K. \& Kurizaki, V. (2009). Reflections on Tools and Strategies Used in the Hawai'i Progress Maps Project. National Center for Assessment/NCIEA.

Duschl, R. A., H. A. Schweingruber, \& Shouse, A.W. (2007). Taking science to school: learning and teaching science in grades K-8. Washington, D.C., National Academies Press.Kennedy, C., \& Wilson, M. (2007). Using Progress Variables to Interpret Student Achievement and Progress. Berkeley: Berkeley Evaluation \& Assessment Research (BEAR) Center.

Michaels, S., Shouse, A.W. \& Schweingruber, H.A. (2008). Ready, set, science: putting research to work in K-8 science classrooms. Washington, D.C. National Academies Press.

Mohan, L., Chen, J., \& Anderson, C. W. (2009). Developing a Multi-Year Learning Progression for Carbon Cycling in Socio-Ecological Systems. [Article]. Journal of Research in Science Teaching, 46(6), 675-698.

National Research Council. (2011). A Framework for K-12 Science Education: Practices, Crosscutting Concepts, and Core Ideas. Washington, D.C. National Academies Press.

Smith, C. L., M. Wiser, et al. (2006). "Implications of Research on Children's Learning for Standards and Assessment: A Proposed Learning Progression for Matter and the Atomic-Molecular Theory." Measurement: Interdisciplinary Research and Perspectives 4(1-2): 1-98.

Wilson, M. (2009). Measuring progressions: Assessment structures underlying a learning progression. Journal of Research in Science Teaching, 46(6), 716-730.

Wiser, M., C. L. Smith, et al. (In Press). Learning Progressions as tool for curriculum development: Lessons from the Inquiry Project). Learning Progression in Science. A. Alonzo and A. Gotwals, Sense Publishing. 


\section{Table of Contents}

Narrative for Hypothetical Learning Progression for

Structure, Properties \& Transformation of Matter

Table for Hypothetical Learning Progression for

Structure, Properties \& Transformation of Matter

Narrative for Hypothetical Learning Progression for

Flow of Matter \& Energy in Ecosystems

Table for Hypothetical Learning Progression for

Flow of Matter \& Energy in Ecosystems 100

Appendix A: Consultants Working on Hypothetical Learning

Progressions

Appendix B: List of Science Practices and Design Principles to use in Developing Hypothetical Learning Performances

Appendix C: Relationship Between NRC Framework and Learning Progression for Structure, Properties \& Transformation of Matter

Appendix D: Relationship Between NRC Framework and Learning Progression for the Flow of Matter \& Energy in Ecosystems 155 


\section{Narrative: \\ The Hypothetical Learning Progression for Structure, Properties and Transformation of Matter}

This paper provides an overview of the hypothetical learning progression (HLP) for the Structure, Properties, and Transformation of Matter and a guide to the accompanying table in which the details of the HLP are presented. We also will identify the core ideas from the NRC Framework that are addressed in this HLP and explain the reasoning behind the structure of the table. Additionally, we will summarize three key aspects of the HLP included in the table representation:

0 the key features of students' explanations or explanatory models at different levels of the HLP, and changes in those explanations from level to level,

o useful or productive ideas and experiences that may help leverage student progress from one level of understanding to another, and

0 trends and changes in learning performances and experiences across the levels.

The paper ends with a highly simplified and annotated outline of these three features.

Section I of the paper provides more detail about how and why we developed each part of the HLP table, including the research used to develop the HLP, and Section II lays out the storyline (or big picture overview) of the HLP table.

\section{Developing the HLP}

The HLP for the Structure, Properties and Transformation of Matter is based on the Framework for K-12 Science Education developed by the National Research Council (National Research Council, 2011). In order to reconcile the evidence we found in the research literature about students' progression through models of matter with the core concepts identified in the NRC Framework we had to combine some of the latter [specifically ideas PS1.A and PS1.B, and parts of PS2.B and PS3.A, in the NRC framework]. As a consequence, the ideas in the HLP are organized somewhat differently from those presented in the NRC Framework or past standards documents. A close comparison of our progression with the NRC Framework reveals that most of the ideas found in Framework are present in our progression, albeit with some elaborations and a few additions or revisions. The difference in how we organized the 
content reflects our desire to represent the coherence we observe in the explanatory models that students are likely to use at different levels. Appendix $C$ illustrates the relationship between the NRC Framework and the explanations or models we identified in the HLP.

\section{The Research}

Several types of research are helpful in developing HLPs. Most directly helpful are empirical studies that document how students' ideas develop over time, how students demonstrate and apply their knowledge, and what experiences or ideas might support the development of new understandings. Studies by Johnson (1998, 2000, and 2002), Stephens \& Delgado et al. (2010), Wiser et al. (2011), and Merritt (2010) fall into this category and were used in this work. Design experiments that examine the relations between the design of a learning ecology, including the particular tasks, tools, forms of argument, and teacher orchestration of these instructional elements and what students learn are also useful. Studies by Merritt (2010), Krajcik \& McNeill et al. (2008), Papageorgiaou and Johnson (2005) and Wiser et al. (2011) fall into this category.

\section{Student Explanations in the HLP}

Our HLP identifies the conceptual models or explanations that, with instruction, students are likely to develop and apply to understanding the structure, properties and transformation of matter. For the purpose of clarity, the structure and properties of matter ideas are combined into one strand in the HLP and are found in the white background sections of the table, while the transformation of matter ideas form another strand and are found in the grey background sections. Using appropriate models of the structure of matter students can describe, predict, and explain a wide range of properties of matter; they can explain that liquids and gases both have weight and take up space or why liquids of one substance may have a higher boiling point than another. The transformations of matter strand includes both physical transformations (i.e., phase changes) and chemical changes (i.e., chemical reactions) as it is important that students consider both types of transformations if they are to explain and predict the variations in matter observed in nature. For example, a student should be able to explain why a physical change such as sugar dissolving in water does not result in a new substance, but a chemical change such as oxygen reacting with iron does. Models that encompass both the structure of matter and its transformations will describe and explain more phenomena than structural models alone. Thus, from a learning and college readiness perspective, combining these strands into one HLP seems worthwhile. 
Our team of consultants considered the most rigorous research in this domain to identify the types of explanatory models students might develop during their school career as they relate to the structure, properties, and transformations of matter. Most of this research focuses on explanations that young children provide, along with some attention to the explanations and models that students use in the middle grades. However, we had to go beyond the research evidence and draw on our collective experience to suggest the kinds of explanations we would expect to observe in upper middle grades and in high school, adding ideas about attractive forces and energy as they apply to collections of molecules. We did this by anticipating that an early rudimentary understanding of these ideas would support the development of more sophisticated models later in high school. With regard to this, it is notable that the NRC framework includes concepts of forces and energy at the middle grade level in their descriptions of some other core ideas (outside of PS1.A and PS1.B) and thus these additions are not necessarily inappropriate. As we noted in the Preface there is much less evidence on how students' thinking actually develops with instruction at more the advanced levels, so our descriptions of those levels, as in the upper middle grade example, represent hypothetical approximations of what we would expect to find and these hypotheses are heavily influenced by the conceptual structures of the scientific disciplines themselves. Whether and how these structures come to be represented in students' thinking are questions that will require further research and instructional design, but we offer them as first approximations.

\section{Selection of Learning Performances}

Our HLP for the Structure, Properties, and Transformation of Matter also includes examples of the kinds of learning performances that students at each level are likely to be capable of demonstrating and the kinds of learning experiences that would support students in advancing through these levels. We have developed schemes for systematically designing examples of learning performances and clustering science practices to ensure that all the practices are represented in the examples (see Appendix B). We have applied these design and clustering schemes to generate the set of learning performance examples shown in our HLP. These are only samples of all the possible performances that might be imagined, but they provide an appreciation of the nature and level of sophistication that students can reach at the level being described. Again, as noted in the Preface, students' sophistication in the application of scientific practices also can be described in learning progression terms, and the learning performances presented in the HLP reflect intersections between the relevant 
conceptual and practices progressions. Ideally learning performances should be selected based on knowledge of the kinds of experiences students are likely to have been exposed to during instruction, and the criteria for judging their performances on both concepts and practices should reflect the levels that those instructional experiences have exposed them to, and have expected from them. We acknowledge that not all the learning performances in this HLP meet these criteria - partly because there are few such well-studied learning performances available to draw from. Nevertheless many of the learning performances presented in the HLP align with assessments or classroom tasks that have been used with students at the levels indicated in the progression (in particular at the elementary and middle school levels) so we are reasonably confident that students can productively engage the set of sample learning performances assigned to each level in the progression.

\section{The HLP Storyline}

An overview of the storyline of the HLP is provided below and can be used in conjunction with the table to build a clear understanding of the progression.

The HLP for the Structure, Properties, and Transformation of Matter describes five levels of explanatory models that students would move through in an ordered sequence:

- A macroscopic compositional model of matter in which matter is something that can be touched or felt, objects can be broken down or divided into smaller parts and the parts still maintain their material identity (e.g., tiny chunks of plastic object are still plastic), and students can, if asked, imagine these samples or chunks as being composed of smaller equal sized units; only liquids and solids and physical reshaping or cutting are investigated and explained at this level.

- A microscopic compositional model of matter in which matter is still seen as something that can be touched or felt, but students can begin to conceive of objects or materials as being composed of very tiny pieces that one needs sensitive instruments to detect; liquids and solids and grinding and melting are investigated at this level. 
- A particle model of matter in which all matter (be it in a solid, liquid, or gas form) is comprised of tiny particles that are in constant motion; gases can be explained and investigated now, as well a phase changes.

- An atomic-molecular model of matter in which particles are conceived of as being either atoms or collections of atoms forming molecules that are in constant motion, and attractive forces and energy influence how the molecules interact with each other; chemical change can now be explained and investigated along with new properties requiring an understanding of interactions between molecules.

- A subatomic model of matter in which atoms are conceived of as having a substructure composed of charged particles that influence interactions within and between molecules and consequently the structure, properties and transformation of matter. Students at this level have explanations with clearer mechanisms for why atoms and molecules behave the way they do and have more refined explanations for chemical and physical transformations of matter.

We emphasize that these levels, which are defined by the changes in the sophistication of the conceptual models that students may develop, are dependent on instruction. They are not likely to develop without support from teachers or curriculum materials providing students with learning experiences that expose them to the phenomena and the explanatory models and require them to use scientific practices in ways that will prepare them to demonstrate the kinds of learning performances expected at each level. For this HLP a key task is for students to reflect on their knowledge of what matter is and what happens when it changes (i.e. during physical or chemical transformations), and it is anticipated that students will revise their conceptions of the nature and behavior of matter as they are asked to test their assumptions against new evidence and observations. Teachers have an important role in providing feedback about what students' models can explain and predict well, and what they cannot explain or predict well. With such support, students might then be able to revise their conceptions in ways that would then reflect the progression we are describing.

\section{Useful or Productive Ideas}

A learning progression perspective is consistent with a developmental perspective on learning in that both emphasize that students learn by integrating new ideas with preexisting ideas. Developers therefore attempt to identify the early ideas (perhaps 
incomplete or partially incorrect ones) that students bring to school so that they can build on them to develop new conceptions. In light of this, the HLP developed for the structure, properties and transformation of matter identifies useful or productive ideas or experiences at each level that might leverage moving to another level.

The outline provided below highlights the key features of the explanations students are likely to offer at each level to account for phenomena related to the structure, properties and transformation of matter as well as some of the useful or productive ideas or experiences at each level that might support consolidating their explanations at that level or moving to the next one. It also includes notes on the sample of learning performances provided across the levels.

Without going into great detail, we have identified a few ideas that students are likely to embrace, which while they are "wrong" or incomplete in some sense, may nevertheless serve as levers or stepping stones to developing more sophisticated models of matter. For example, early on (level 2) the idea that objects or materials can be divided into very small units (whose presence can only be detected with sensitive instruments) that maintain their material identity and maintain their weight across physical reshaping or dividing can leverage student thinking about the particle model in level 3. For another example, the particle model (level 3) which doesn't differentiate between atoms and molecules, but nonetheless conceives of particles too small to see even with the aid of a microscope is a stepping stone to thinking about atoms and molecules at level 4 . Identification of these ideas, along with a discussion of the learning performances, provides clues as to how students might move from one level of thinking to another.

\section{Learning Performances and Experiences}

The common element in the set of learning performances and experiences described in the HLP is that students at each level are asked to explain, investigate, and argue about broader classes of phenomena than they previously could. The phenomena become more complex and more varied at each level; for example, at levels 1 and 2 phenomena involving only physical transformations of solids and liquids are explained or investigated, at level 3 physical transformations involving gases are explained or investigated; and, then at level 4 phenomena involving chemical reactions are explained and investigated. As students progress across the levels, they are able to provide increasingly sophisticated explanations for these phenomena. Students functioning at lower levels would be unlikely to be able to offer similar explanations for the phenomena. 
The increasing sophistication of students' learning performances also highlights a pattern in their thinking across the levels: students' models of matter shift from being solely descriptive to being more mechanistic. This is perhaps most evident in the shift between level 3 and level 4 . Students functioning at level 3 understand the particle model and can provide descriptive explanations for what happens when gases expand, but the model does not explain why the particles behave that way. To address this, ideas about the attractive forces between molecules and the kinetic energy of the collection of molecules must be understood. Once these mechanisms are understood, students can engage in more sophisticated arguments about why some liquids have a higher boiling point than others liquids.

\section{The Outline of the HLP}

Since the storyline of the HLP for the Structure, and Properties and Transformation of Matter may be difficult to follow in the table, we present it here in a more simplified form. The outline highlights the key features of the explanatory model at each level, some key ideas within each model that serve as leverage to the next level, and some notes on how ideas are being used in more sophisticated ways as reflected in the learning performances. The table includes a section that highlights "what is changing in students' thinking" and that section provides a more complete description of the changes between adjacent levels.

\section{Level 1: Macroscopic compositional model of matter}

- Key features of model:

- Students conceive of matter as that which you can touch, feel, see, and smell.

- Students' understanding of matter is limited to solids and liquids and to physical changes like reshaping or dividing.

- Students come to conceive of materials or objects as being composed of smaller equal sized units - a compositional model develops in which "amount" is proportional to the number of units (e.g. larger samples are made of more equal sized units than smaller samples).

- Ideas of leverage to next level:

- Matter maintains its material identity across different physical reshaping events (e.g. silver shaped into different objects).

- Liquids and solids have something in common: they both have weight and occupy space.

- Understanding aggregates: objects can be broken down into smaller pieces, detectable with tools like a magnifying glass or scales, which are still the same materials and have weight [but aggregates at this level do not include powders].

- Learning performances and experiences: 
- Phenomena students investigate or explain are limited to solids or liquids [no gases], and reshaping or dividing into smaller pieces [no grinding]; also limited to scales that students can see with their own eyes [or perhaps with just a magnifying glass]

- In explaining differences in materials, students can use macroscopic intrinsic properties [e.g. color, hardness, smell].

- In designing investigations students can account for the "amount" of material being conserved during reshaping by using a compositional model [i.e. they can apply proportional reasoning in which bigger or larger objects are perceived as being made of more equal size units - and something that is smaller has fewer equal size units; they know that reshaping involves spatially rearranging the units, but not changing their number].

- In designing investigations students can use intrinsic macroscopic properties of materials to explore different materials (such as the color and luster of metals) [before, in pre-K, students might not have considered using material identity to classify objects].

- In designing investigations and constructing arguments students should apply experiences they have had with using scales for weighing objects and materials [instead of using heft which pre-K students might do] because they should have come to realize that scales may be able to detect small amounts that a human hand cannot.

\section{Level 2: Microscopic compositional model of matter}

- Key features of model:

- To these students, matter is still limited to solids and liquids, but they can think of matter at a microscopic level (e.g. needing sensitive tools like microscopes to detect).

- Students integrate the amount of material, weight, volume, and material identity via the microscopic compositional model to allow for a deeper understanding of what it means for something to be "composed of material" (in other words any sample of a liquid or solid is made of a specific type of material, even very tiny samples, and any sample of liquid or solid can be conceived of as being composed of many smaller equal sized units) and therefore solids and liquids are viewed as deeply similar.

- Students realize that both amount and identity of material are conserved and weight does not change during grinding (division into very small pieces), melting, freezing, and dissolving -- and that volume does not change during grinding.

- Ideas of leverage to next level:

- A tiny bit of solid or liquid can be detectable with sensitive instruments.

- Matter has weight and volume and maintains its material identity when divided into very small units.

- Learning performances:

- Phenomena that students investigate or explain are still limited to solids and liquids, but they can explore smaller scales such as tiny grains of sand or powder that can be viewed with a microscope or that can be detected with very sensitive scales; physical division including grinding, melting, freezing and dissolving are explored [before, chopping or dividing at scales that are visible to the eye were explored, or perhaps observed with simple magnifying glasses]. 
- Phenomena that students predict and explain can also involve a wider range of materials.

- In explaining and constructing arguments for differences or similarities among different objects, students should be able to consider material identity, weight, amount of material and volume as central properties of solid and liquid samples, and know that weight and volume are proportional to amount of material; they should also be able to apply both conservation of material identity and amount of material, and weight constancy to physical transformations (grinding, melting, freezing, dissolving).

- In designing investigations, students can draw upon quantitative reasoning and conservation of material identity and take into consideration some non-perceptual intrinsic properties of materials (e.g., melting point) to justify designs.

- In designing investigations and constructing arguments, students should more consistently consider using scales to examine weight [instead of using heft] because they should realize scales may be more sensitive and accurate.

\section{Level 3: Particle model of matter}

- Key features of model:

- For these students, matter is composed of very tiny particles that cannot be seen even with microscopes; those tiny particles are in constant motion and their arrangement and motion are different when a particular substance is in a solid, liquid, or gaseous state.

- Students can explain the behavior of gases and see liquids, solids, and gases as fundamentally similar because they all have weight and take up space.

- Students can explain a broader range of transformations including boiling and evaporation and better account for transformations such as mixing and dissolving [but cannot explain chemical transformations well yet].

- Ideas of leverage to next level:

- The idea that matter is composed of tiny particles that cannot be seen with the unaided eye is a stepping stone to thinking about particles being made up of atoms or molecules

- The idea that there is empty space [i.e. a vacuum] between particles further elaborates what a particle is and can account both for the behavior of particles when they mix, and for when they come closer together or spread apart during contraction and expansion.

- Learning performances and experiences:

- Phenomena that students can explain, investigate, and argue about now include gases [which were not considered before]; therefore students can explain and explore boiling, evaporation, expansion of gases.

- Phenomena that students explain or investigate using the particle model are more complex-for instance, dissolving, or expansion of gases upon heating.

- In explaining and constructing arguments about the properties or transformation of matter, students use and apply the particle model, but explanations are essentially descriptive and lack deeper mechanisms to explain why particles behave as they do. 
- In designing investigations and constructing arguments, students can now draw upon a wider range of intrinsic properties [e.g. density and boiling point] to distinguish and identify different materials.

\section{Level 4: Atomic-molecular model of matter (with attractive forces between molecules and energy associated with collection of molecules, formerly "particles")}

- Key features of model:

- Students conceive of matter as composed of atoms which form molecules [formerly all "particles"].

- Students now consider attractive forces and energy to explain the behavior of molecules.

- Students conceive of chemical transformations as a rearrangement of atoms to form new molecules.

- Ideas of leverage to next level:

- The idea of atoms that make up matter is a prerequisite to thinking about subatomic structures.

- The ideas of attractive forces and changes in energy associated with collection of molecules can be used to build more sophisticated ideas about energy changes and attractive and repulsive forces between atoms.

- Learning performances:

- The phenomena students explain, investigate, and argue about now include chemical reactions; students also begin to explore a wider range of intrinsic properties that require ideas about attractive forces or energy to explain (e.g. differences in boiling point).

- In explaining or constructing arguments, students can use and apply the concepts of atoms and molecules [as opposed to the general notion of a small particle applied before]; for instance, students account for conservation of matter during transformations by paying attention to the numbers and types of atoms [whereas before students could only think of the total number of particles or conceive of conservation of macroscopic weight].

- In explaining physical transformations, students can offer more detail about the mechanisms to account for them by including attractive forces and energy (e.g. reasons for why "particles" behave as they do).

- In designing investigations, students use a greater variety of intrinsic properties of materials, including reactivity with specific elements or those involving ideas of attractive forces between molecules.

- In critiquing others models or explanations, students can consider whether other students have articulated mechanisms of matter transformation events, as opposed to offering mere descriptive accounts for what happens during a transformation. In other words, students would look for the inclusion of attractive forces in the model or explanation because this would reflect a clearer mechanism for explaining why particles behave as they do during physical changes such as boiling. A model or explanation without attractive forces would be more descriptive in nature. 


\section{- Level 5: Sub-atomic model of matter}

- Key features of model:

- Students conceive of atoms as having a substructure of negatively charged electrons, positively charged protons, and neutrons with no net charge, with protons and neutrons constituting a nucleus with electrons moving around it.

- Students can think about the attractive forces between atoms and molecules as involving positive or negative charges (associated with subatomic particles) and consideration of these interactions can be found in students' explanations for the structure, properties, and transformation of matter.

- Students' explanations for some properties of matter, such as polarity, begin to include a clearer and more refined mechanism.

- Students' explanations for the structure, properties, and transformation of matter include the general idea that a collection of atoms moves towards a state of lower energy.

- Learning performances:

- The phenomena students explain, investigate, reason and argue about include properties like polarity and charge (which represent a new set of properties that are common in the natural world).

- In explaining or constructing arguments, students can use and apply ideas of sub-atomic charged particles and the electrostatic interactions that result from their interactions [as opposed to general notion of "attractive forces" noted before].

- In designing investigations, students draw upon more sophisticated models of matter or examine and measure more specific features of transformations (e.g. examine factors that influence chemical reactions) and therefore are examining more sophisticated mechanisms underlying chemical transformation.

- In critiquing models or explanations, students can consider whether they offer clearer or more refined mechanisms to explain the structure and transformation of matter, in particular the consideration of electrostatic forces and the tendency of systems of atoms to move toward a state of lower energy [as opposed to considering attractive forces in a generic sense, which is a more rudimentary mechanism]

When reading the table, some levels of thinking (level 4 and 5) appear particularly dense. They include the strands for the structure and properties of matter as well as the transformation of matter (transformation of matter includes sections for both chemical and physical transformation). At level 4 students are developing ideas that allow them to distinguish chemical from physical transformations, but at the same time their models of how matter behaves incorporate more sophisticated mechanisms to account for the ways molecules interact with each other. These changes in thinking allow students to explain a wider range of phenomena and move from descriptive explanations to explanations with more refined mechanisms for the physical transformations of matter. We believe these ideas can support the development of more complex explanations 
that can account for the interactions between and within molecules and atoms. Level 5 in the table is the continued refinement of those models and explanations and therefore maintains these same strands. 
Figure 1. Snapshot of some of the different sections found in the table for this HLP

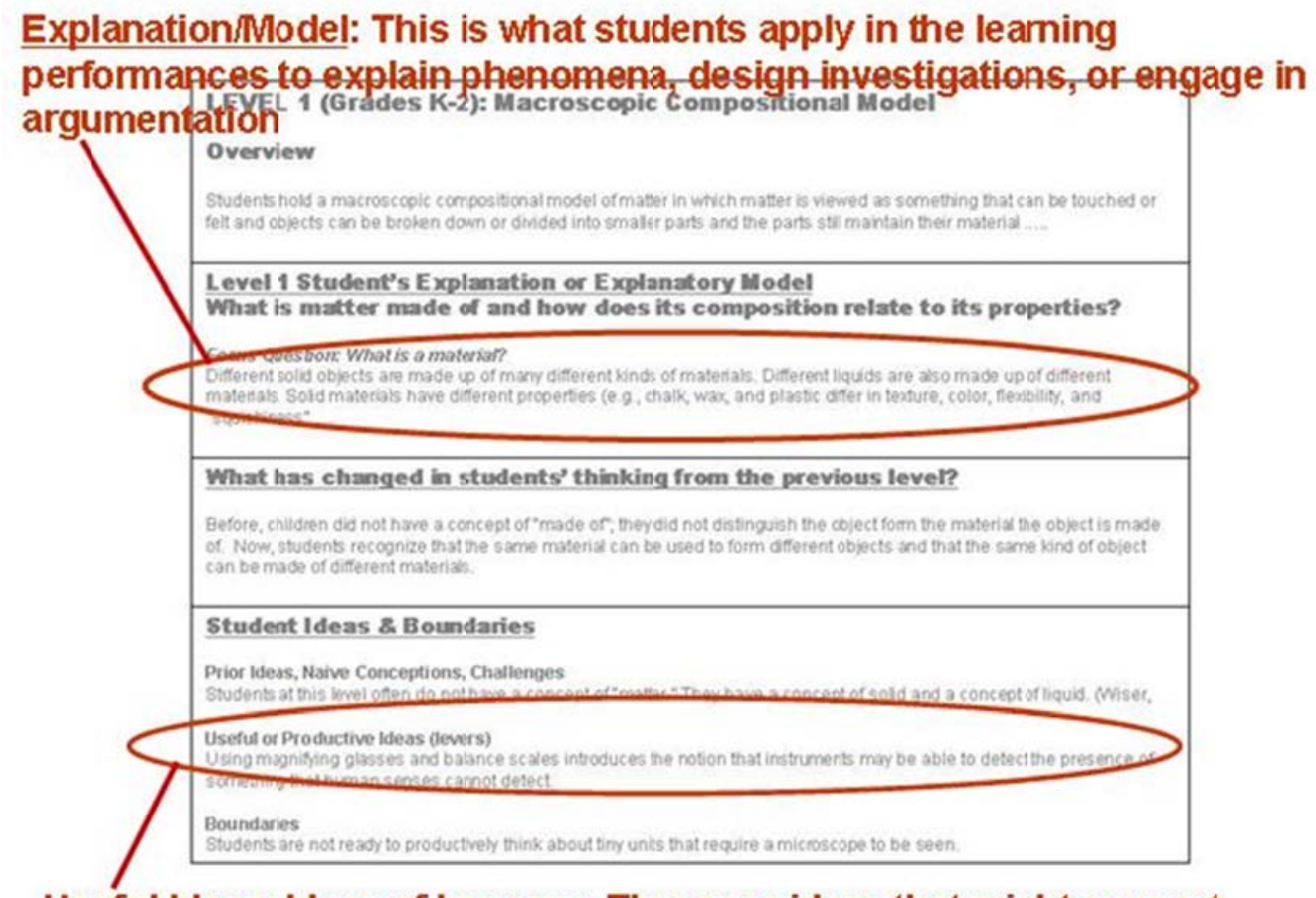

Useful Ideas: Ideas of leverage. These are ideas that might support development of ideas within a level or at the next level

\footnotetext{
Rationale and Notes

- Students at this level tend to focus on things they can see, feel, and touch and do not spontaneously condider colects and phenomena at smaller scales; therefore, eath exploration of matter should take adrantage of this tendency. Moving too earty to the microscopic or nanoscopic levels of organization would be an unproductive use of classtocm instruction time.

How students demonstrate and apply their knowledge (as reflected in Learning Performances)

Pehencmena that students at this level can imestigate orexplain are imited to solids or Iquids. In addition, samples to (imestigale are Imited to a scale that students can see with their own eves for perh aps with just a magnifying glass] oln explaning differences in oblects or materiaks, students can use macros copic intrinsic propenties le o color, hardness: smeli).

oln desigrina imestigations. studens, can account for "amount of matertal being conserved during reshasing by using a compositonal mode

Sample Learning Experiences \& Learning Performances (LE/.P)

Explaining, predicting, developing explanatory models (cluster 1)

LELP 1. Explain what is smilar about two diterenty shaced objects made of the same material (e.g. a siver spocn and an stiver cupl

Criteria:

CWhile many students should be able to say that any two objects have similar properties like color and luster, a more scophisticated idea would be that both are made of the same material because ther have similar properties, even though thes thime dinarents

Designing imvestigations, collecting, representing, and analyzing data (cluster 2 )

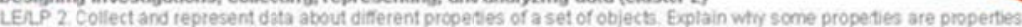
of objects and others are properties of materiats Costeria

Stris

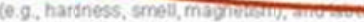

Learning performances: These reflect how knowledge is applied in context of specific science practices. These performances grow in sophistication as explanations or models change
} 


\section{References}

Johnson, P. (1998). "Progression in children's understanding of a 'basic' particle theory: a longitudinal study." International Journal of Science Education 20(4): 393-412.

Johnson, P. (2000). "Children's understanding of substances, Part 1: recognizing chemical change." International Journal of Science Education 22(7): 719-737.

Johnson, P. (2002). "Children's understanding of substances, Part 2: explaining chemical change." International Journal of Science Education 24(10): 1037-1054.

Krajcik, J., K. L. McNeill, et al. (2008). "Learning-goals-driven design model: Developing curriculum materials that align with national standards and incorporate project-based pedagogy." Science Education 92(1): 1-32.

Merritt, J. (2010). Tracking Students' Understanding of the Particle Nature of Matter. Ann Arbor, University of Michigan. Doctoral Dissertation.

National Research Council (2011). A Framework for K-12 Science Education: Practices, Crosscutting Concepts, and Core Ideas. Washington D.C.: National Academies Press

Papageorgiou, G and Johnson, P (2005). Do Particle Ideas Help or Hinder Pupils' Understanding of Phenomena? International Journal of Science Education 27(11)1299-1317.

Stevens, S. Y., C. Delgado, et al. (2010). "Developing a Hypothetical Multi-Dimensional Learning Progression for the Nature of Matter." Journal of Research in Science Teaching 47(6): 687-715.

Wiser, M., C. L. Smith, et al. (In Press). Learning Progressions as tool for curriculum development: Lessons from the Inquiry Project). Learning Progression in Science. A. Alonzo and A. Gotwals, Sense Publishing. 


\section{Structure, Properties, and Transformation of Matter: Level 1 White background-structure \& properties of matter; Grey background - transformation of matter}

\section{LEVEL 1 (Grades K-2): Macroscopic Compositional Model}

\section{Overview}

Students hold a macroscopic compositional model of matter in which matter is viewed as something that can be touched or felt and objects can be broken down or divided into smaller parts and the parts still maintain their material identity (e.g., tiny chunks of a plastic object are still plastic). Samples of materials can be imagined as being composed of smaller equal sized units. At this level only liquids and solids and physical reshaping or cutting are considered and explained in the model.

\section{Level 1 Student's Explanation or Explanatory Model}

What is matter made of and how does its composition relate to its properties?

\section{Focus Question: What is a material?}

Different solid objects are made up of many different kinds of materials. Different liquids are also made up of different materials.

Solid materials have different properties (e.g., chalk, wax, and plastic differ in texture, color, flexibility, and "squishiness" [malleability]). Liquid materials also have different properties (e.g., liquid water, olive oil, and rubbing alcohol differ in taste, smell and ease of flow). Some materials can be found in nature; others are synthetic (made by humans). Different materials are used to make objects with different uses.

When you divide something into smaller pieces it is still the same material (macroscopic compositional model).

(Note: the idea that material identity is conserved upon division is independent of the idea that "weight" is conserved upon division at this level)

\section{What has changed in students' thinking from the previous level?}

[Note: at level 1, these changes are relative to the early understandings that small children are expected to have before entering kindergarten].

o Before, children did not have a concept of "made of"; they did not distinguish the object form the material the object is made of. Now, students recognize that the same material can be used to form different objects and that the same kind of object can be made of different materials.

o Students start developing a concept of material as that which constitutes objects and has specific properties independently of the shape of objects.

o Students at this level will also learn to quantify the "amount" of material an object is made of. This is a first step toward linking amount of material with weight and to volume (in the next level), and to developing the concept of mass in middle school. Students develop a concept of amount of material by applying a compositional model to objects.

o Students develop an objective and extensive concept of weight. Young children only have the concept of heft (i.e. what you can feel in your hand). 


\section{Structure, Properties, and Transformation of Matter: Level 1 White background-structure \& properties of matter; Grey background - transformation of matter}

o Students now realize that instruments [like simple magnifying glasses] can improve their ability to detect the presence of small pieces of material, even when they are too small to see with the naked eye.

o Students start seeing deep similarities between liquids and solids. Before, they considered them to be extremely different. In this grade range, they learn that liquids, like solids, are materials with specific properties, that they have weight, and that they can be divided into smaller quantities.

\section{Student Ideas \& Boundaries}

\section{Prior Ideas, Naive Conceptions, Challenges}

Students at this level often do not have a concept of "matter." They have a concept of solid and a concept of liquid. They see liquids and solids as being extremely different (Wiser, O'Conner et al., 1995)

\section{Useful or Productive Ideas (levers)}

Using magnifying glasses and balance scales introduces the notion that instruments may be able to detect the presence of something that human senses cannot detect. This is a precursor to learning that objects that cannot be seen with the unaided eye exist and have weight.

Since preschoolers believe that solids and liquids are very different kinds of things (Carey 1991), K-2 students should learn that solids and liquids have important properties in common: they have weight and they occupy space.

Aggregates (like sand or powders) are a bridge between solids and liquids since they can be poured and fall to the bottom of containers, like liquids, but are made of small pieces of solid materials. Observing, classifying, and measuring liquids as well as solids will help students build the category "solid and liquid" --a stepping stone for the concept of matter taught in the next grade band, and for understanding that the same material can exist in liquid and solid form (Terc, 2011).

Preschoolers do not have a concept of material and focus on whole objects instead of the materials they are made of (e.g., they may understand that a silver spoon is shiny and hard, but do not realize the spoon has these properties because it is made of silver) (Bloom 2002; Wiser and Smith 2008). Thus, $\mathrm{K}-2$ students should learn to differentiate objects from the materials they are made of, and distinguish properties that characterize objects (e.g., size,

function) from those that characterize materials (e.g., hardness, "squishiness", smell).

Hardness needs careful attention. It is an important property of solid materials, and it is salient to students (i.e. wood is hard, foam is soft). However, when a hard solid is ground, it is no longer hard perceptually; therefore, for many students it does not appear to be the same material (Dickinson 1987).

\section{Boundaries}

Students are not ready to productively think about tiny units that require a microscope to be seen.

It would be too soon to talk about a particular material existing in different phases. The focus should remain on material identity.

Density is a complicated idea that should not be introduced at this time. However, the concepts of "heavy for size" or "heavier for the same volume" can be developed at this grade band. 


\section{Structure, Properties, and Transformation of Matter: Level 1 White background-structure \& properties of matter; Grey background - transformation of matter}

\section{Rationale and Notes}

o Students at this level tend to focus on things they can see, feel, and touch and do not spontaneously consider objects and phenomena at smaller scales; therefore, early exploration of matter should take advantage of this tendency. Moving too early to the microscopic or nanoscopic levels of organization would be an unproductive use of classroom instruction time. Experiences with instruments such as simple magnifying lenses can begin to expand their knowledge of the size of objects below what is visible to the naked eye.

o Students should also focus on developing a notion of material identity (i.e., objects are made of different materials with different properties) and that objects have "weight" even in the case of very small objects or low density materials. These notions of material identity and weight can be used as leverage to understand later that matter is made of tiny particles that one cannot see which have unique identities and weight (and, in middle school, mass).

\section{Notes on how students demonstrate and apply their knowledge (as reflected in Learning Performances)}

o Phenomena that students at this level can investigate or explain are limited to solids or liquids. In addition, samples to investigate are limited to a scale that students can see with their own eyes [or perhaps with just a magnifying glass].

o In explaining differences in objects or materials, students can use macroscopic intrinsic properties (e.g. color, hardness, smell).

o In designing investigations students, can account for "amount" of material being conserved during reshaping by using a compositional model.

- In designing investigations students can use intrinsic macroscopic properties of materials to explore different materials (such as the color and luster of metals) [before, in pre-K, students might not have considered material identity to classify objects].

o In designing investigations and constructing arguments students should consider using scales to examine weight [instead of using heft, which preK students might do, because they should realize scales may be able to detect small amounts that a human hand cannot].

\section{Sample Learning Experiences \& Learning Performances (LE/LP)}

\section{Explaining, predicting, developing explanatory models (cluster 1)}

LE/LP 1. Explain what is similar about two differently shaped objects made of the same material (e.g., a silver spoon and an silver cup). Criteria:

o While many students should be able to say that any two objects have similar properties like color and luster, a more sophisticated idea would be that both are made of the same material because they have similar properties, even though they have different shapes.

Designing investigations, collecting, representing, and analyzing data (cluster 2) 


\section{Structure, Properties, and Transformation of Matter: Level $\mathbf{1}$ White background-structure \& properties of matter; Grey background - transformation of matter}

LE/LP 2. Collect and represent data about different properties of a set of objects. Explain why some properties are properties of objects and others are properties of materials.

Criteria:

o Students' data should be about different properties of objects (e.g., size, weight, shape) and different properties of materials (e.g., hardness, smell, magnetism), and labeled as such.

o Students should articulate that properties inherent in materials are independent of the shape and size of an object whereas others such as weight depend on size. They should learn to focus on inherent, rather than accidental properties, of materials (e.g., paint on an object that is not inherent to the object). K students are likely to sort objects by one characteristic at a time, first in terms of object identity then in terms of materials.

LE/LP 3. Design experiments to determine the weight of different objects. Present and explain findings. Criteria:

o Students should learn not to rely on heft to compare weight because heft is imprecise and can be misleading. They should use a pan balance scale and use a weight line to represent their data (students would be expected to array the objects themselves according to weight).

o Students will notice that, for a given material, weight increases with size. They should explain that bigger objects are heavier because they are made of more material.

\section{Constructing scientific arguments (cluster 3)}

LE/LP 4.Explain and justify why two unknown objects that have roughly the same size have different weights.

Criteria:

o Students should notice and say that the weight difference is not due to size. The hypothesis that one object is hollow is plausible and may be offered, but it is not sophisticated. A more sophisticated hypothesis is that they are made of different materials, one material being heavier than the other for equal volumes.

LE/LP 5. Critique a student's claim that a small piece of metal weighs nothing because "it feels like nothing." Design an experiment to support the argument.

Criteria:

o Students should argue that heft is not a reliable measure of weight and that even small pieces of material have weight. They should use a scale to demonstrate that the piece of metal has weight. If the scale does not detect the weight of the piece, they should argue that a more sensitive scale would; or they can use a number of similarly small pieces to show that taken together they have a measurable weight and reason/argue that dividing that total by the number of pieces shouldn't get you to zero ("quantitative reasoning"), just to small or very light.

Learning Performances informed by work of Wiser and colleagues with elementary students (TERC, 2011; Wiser, Smith et al., In Press) 


\section{Structure, Properties, and Transformation of Matter: Level 1 \\ White background-structure \& properties of matter; Grey background - transformation of matter}

\section{Level 1 Student's Explanation or Explanatory Model (physical transformations)}

What is taking place when matter changes?

Focus Question: What happens to material when objects change shape?

If you cut up an object into pieces, the identity and the amount of material do not change. For example, if you cut up a chunk of wood into smaller pieces, its pieces are still wood, and the amount of wood stays the same. The weight of all the pieces together is the same as the weight of the original object. Similarly, if you reshape an object made of clay or wax, the amount of material and its weight stay the same. This is true for samples of liquids as well.

\section{What has changed in students' thinking from the previous level?}

[Note: at level 1, these changes are relative to the early understandings that small children are expected to have before entering kindergarten]

0 Before kindergarten, children find it hard to consistently believe that material identity is maintained when an object is cut into pieces. Some students do, but only when the pieces are big enough to keep all the perceptual properties as the object they came from. The shift in this grade band is for students to expand their understanding of material identity conservation to all contexts in which solids are divided into pieces.

- At this level students should also develop an understanding that "amount" of material is conserved when objects are reshaped or cut into pieces. Using the macroscopic compositional model of material, they can visualize pieces being spatially rearranged without their total number changing. They should also understand that weight does not change when a solid sample is cut into pieces.

\section{Student Ideas \& Boundaries (physical transformations)}

\section{Prior Ideas, Naive Conceptions, Challenges}

Students at this level judge things perceptually and trust their senses. That is why they believe amount of material and weight change when shape changes-when a ball is flattened into a pancake, it feels lighter. The concept of amount of material, based on the compositional model, requires overcoming perceptual information and thinking of amount in terms of number of units. Linking counting to measurement is a challenge in this grade range.

\section{Useful or Productive Ideas (levers)}

At this level, students learn that the type of material a solid object is made out of does not change when it is cut into pieces. In other words it keeps its perceptual properties. At the next level, this serves as a lever for learning that material identity does not change when a solid is ground or melts.

Students at this grade band also learn to quantify the amount of material an object is made of and to conserve it across reshaping and cutting objects. This is a first step toward linking amount, weight and volume in the next grade range, and the concept of mass in middle school.

\section{Boundaries}

It would be too soon to talk about physical changes such as melting or freezing. 


\section{Structure, Properties, and Transformation of Matter: Level 1 White background-structure \& properties of matter; Grey background - transformation of matter \\ Rationale and Notes}

o The transformations addressed at this level (reshaping and cutting) preserve material identity and therefore are precursors of grinding, phase changes and dissolution, but not of chemical changes (in which material identity changes)

o Students at this level will also learn to quantify the amount of material an object is made of. This is a first step toward linking amount, weight and volume in the next level, and the concept of mass in middle school. [Note, the term weight is used here even though mass is the correct scientific terms, because weight is something students are familiar with and can use as they explore and make sense of the nature world at the macroscopic level. Moreover, students' exploration of mass is all relative to conditions on earth at this level so they do not need to consider other gravitational fields.]

\section{Notes on how students demonstrate and apply their knowledge (as reflected in Learning Performances)}

o Phenomena that students investigate or explain are limited to solids or liquids; also limited to scales that students can see with their own eyes [or perhaps with just a magnifying glass].

o In explaining differences in chopped up objects, students can account for "amount" of material being conserved during reshaping by using a compositional model.

\section{Sample Learning Experiences \& Learning Performances (LE/LP) (physical transformations)}

Explaining, predicting, developing explanatory models (cluster 1)

LE/LP 1. Predict and justify whether the amount of material is going to change when an object is reshaped or cut into pieces. Criteria:

o Students should invoke the compositional model of material: an object can be mentally decomposed into equal pieces when it changes shape the pieces are spatially rearranged but their number stays the same.

LE/LP 2. Predict and justify whether or not an object that is chopped up into small pieces is made of the same material as before. Criteria:

o Students' justifications should include a notion of material identity being conserved and that cutting does not change material identity. A sophisticated justification could include the notion that the pieces composed the original chunk, or that one can imagine "putting them back together" to reform the chunk.

Constructing scientific arguments (cluster 3 )

LE/LP 3. Construct an evidence-based argument for why the amount of liquid (measured as either weight or volume) in a container does not change when the liquid is poured into a different-shaped container. Criteria: 


\section{Structure, Properties, and Transformation of Matter: Level 1}

White background-structure \& properties of matter; Grey background - transformation of matter

0 Student should predict that amount does not change if nothing is added or removed and verify their prediction by measuring or weighing it before and after transferring it to a different container. Students could also measure the constancy of volume using graduated containers (e.g., a tall, thin, graduated cylinder; a wide, short beaker; and a conical Erlenmeyer flask, all with volumetric markings).

Learning Performances informed by work of Wiser and colleagues with elementary students (TERC, 2011; Wiser, Smith et al. In Press) 


\section{Structure, Properties, and Transformation of Matter: Level 2 \\ White background-structure \& properties of matter; Grey background - transformation of matter}

LEVEL 2 (Grades 3-4): Microscopic Continuous Mode

\section{Overview}

Students hold a microscopic compositional model of matter in which matter is still seen as something that can be touched or felt, but students can begin to conceive of objects or materials as being composed of very tiny pieces that one needs sensitive instruments to detect. Liquids and solids and grinding (into powders or other fine particulates) and melting are more adequately explained and investigated at this level than other physical transformation or

remodeling events.

\section{Level 2 Students' Explanation or Explanatory Model}

What is matter made of and how does its composition relate to its properties?

\section{Focus Question: What is matter?}

Matter is anything that has weight and takes up space (i.e., has volume); types of matter include solids and liquids. Matter can be imagined to exist in pieces too small to see with the unaided eye, that still have weight, volume, and the same properties as the material they compose. One would need powerful microscopes and sensitive scales to see these tiny pieces and measure their weight, but the model posits that they would exist.

\section{What are some of its states?}

Matter exists as many different materials, many of which can transition back and forth between solid and liquid.

\section{What are some Properties of Materials?}

The temperature at which a material transitions from solid to liquid is characteristic for each material.

Objects made of some materials are heavier for the same volume than objects made of other materials.

\section{What has changed in students' thinking from the previous level?}

o Students have moved from a macroscopic compositional model of material in Level 1, to a microscopic compositional model in Level 2. Both models express the idea that a chunk of material can be divided into smaller pieces, each made of that material and each having weight. Now, however, the pieces can be thought of as being arbitrarily small and are no longer thought of as pieces that were cut from the original material. Each piece also has volume, no matter how tiny. At the previous level students could conceive of such units, only here the units are even smaller. [Note: this model is not about the structure of matter per se, it is a model for quantification and constancy across shape change. Unlike atoms and molecules, the pieces here are "created" by the person who imagines cutting the object into units]

o While students still may have a view of matter as being continuous (i.e., one in which matter can indefinitely be divided and the pieces will maintain the identity and properties of the original material), they now realize that it can exist in pieces so small that one would need extremely sensitive instruments to detect them, such as a microscope (this is not yet a nanoscopic or atomic/molecular scale).

o Students develop a deeper understanding of what it means to be "made of a particular material". Before they interpreted the phrase "made of" as "made from" (i.e. made from a tree) or refer to an object's perceptual properties such as color and luster, but now they understand the phrase to 


\section{Structure, Properties, and Transformation of Matter: Level 2}

White background-structure \& properties of matter; Grey background - transformation of matter

mean that an object is made of a particular material all the way through, and they are able to imagine that an object could be made of tiny pieces of that material- each having weight and volume.

- At the previous level students explored a range of different liquids and solids, but focused on their properties as solid or liquid materials. Now students realize that many materials can exist as either liquid or solid.

o Before, the concept of weight was applied to both objects and materials, but in this grade range students also learn to coordinate weight, size, and material as they differentiate "heavy" from "heavy for its size" and then "heavy for volume" (a precursor to the concept of density).

\section{Student Ideas \& Boundaries}

\section{Prior Ideas, Naive Conceptions, Challenges}

Students may mistakenly believe that solids and liquids are two different types of matter, instead of different states of matter (Stavy, 1991).

At the beginning of this grade range students' judgments are strongly based on perception. They are likely to believe that small pieces of matter weigh nothing and do not occupy space (M. Wiser, personal communication). They are also likely to believe that no piece of matter that is invisible to the naked eye exists, and that many materials change identity when they melt or are ground (M. Wiser, personal communication). Helping them to understand that observations through the unaided senses can be unreliable and to develop other ways to think about the physical world is important. Instruments that help to visualize or detect smaller pieces are helpful for this. (Smith, Wiser, et al, 2006)

\section{Useful or Productive Ideas (levers and stepping stones)}

Achieving the understanding that any tiny piece of a solid or liquid has weight and takes up space is a lever to the concept of gas and the concept of matter as existing in three states, solid, liquid, and gas in the next grade range (Smith, Wiser et al., 2011).

Students need to construct the concept of volume in this grade range in order to acquire the notion that any piece of matter has volume, and the notion of density later on.

The idea of "heavy for its size" is a lever for the concept of density, which is best understood after a particle model is developed (Smith, Snir et al., 1992).

The concept of material achieved in this grade range is a stepping stone toward the concept of substance developed at the next level.

\section{Boundaries}

It is too early to explore gases. Students need a particle model, developed at the next level, to best understand gases.

\section{Rationale and Notes}

It is helpful for students to learn that some properties that are most relevant to identifying materials cannot be assessed perceptually because this is part of developing a more scientific concept of "material" and it will help students establish the conservation of material identity across phase change as a general principle among all physical transformations at the next level.

o The main goal of instruction at this level is to build an understanding of solids and liquids as being deeply similar: any amount of solid or liquid 


\section{Structure, Properties, and Transformation of Matter: Level 2}

White background-structure \& properties of matter; Grey background - transformation of matter

material has weight and volume, including pieces too small to feel or to see with the naked eye. This category of "solid and liquid" is a useful precursor to the concept of "matter." It is also likely to contribute to avoiding the naïve conception that being liquid or solid is an inherent property of a material.

o With the ideas described above in place, students at the next level can begin to think about gases in a more meaningful way - in particular students will be more likely to understand that gases are like solids or liquids since they have weight and volume and therefore are a material. They will also be more likely to count weight and volume as evidence that mass is conserved during phase change.

o Discovering that different materials have different melting points and that objects made of different materials are more or less heavy for their size helps students to differentiate between properties of materials that can be detected with the unaided senses and those that cannot. These experiences with intrinsic properties that are not easily detected with human senses also help students to learn that instruments may be more sensitive and reliable than sensory use.

The idea that some materials are heavier for their size than others is a precursor idea to the idea of density.

\section{Notes on how students demonstrate and apply their knowledge (as reflected in Learning Performances)}

o Phenomena that students investigate or explain are still limited to solids and liquids, but they can explore smaller scales such as tiny grains of sand or powder that can be viewed with a microscope or that can be detected with very sensitive scales.

o Phenomena that students predict and explain also involve a wider range of materials.

o In explaining and constructing arguments for differences or similarities among different objects, students should be able to consider material identity, weight, amount of material and volume as central properties of solid and liquid samples, and know that weight and volume are proportional to amount of material.

o In designing investigations, students can draw upon quantitative reasoning and conservation of material identity to justify designs and take into consideration some non-perceptual intrinsic properties of materials (e.g., boiling point).

o In designing investigations and constructing arguments, students should more consistently consider using scales to examine weight [instead of using heft] because they should realize scales are more sensitive and accurate.

Sample Learning Experiences \& Learning Performances (LE/LP) 


\section{Structure, Properties, and Transformation of Matter: Level 2 White background-structure \& properties of matter; Grey background - transformation of matter}

\section{Explaining, predicting, developing explanatory models (cluster 1)}

LE/LP 1 Explain how solids and liquids are similar.

Criteria:

o Students should note that both have weight and take up space and that both can be divided into smaller units that also take up space and have weight.

LE/LP 2 Construct an evidence-based explanation for whether the tiny pieces of an object that are crushed or ground constitute the total mass of the original object when the all the pieces are added up.

Criteria:

o Student's explanation could rely on evidence from comparing the weights of the ground material and the weight of the solid chunk. Since material identity has not changed and weight is the same, amount of material has not changed.

LE/LP 3. Explain why tiny pieces of material, even invisible ones, exist, have weight and occupy space.

Criteria:

o Students should rely on the compositional model of material: one can mentally decompose a chunk of material into arbitrarily small pieces. If those pieces are small enough they would not be detectable by the naked eye nor even under a microscope. Yet, they have to exist and have weight and volume because the amount of material, weight, and volume of the solid chunk is the sum of the amount of material, weight and volume of the pieces, however tiny the component pieces.

LE/LP 4. Predict whether two equal-sized objects made of different materials and having different weights will cause the level of water in a container to rise by the same amount. Justify the prediction.

Criteria:

o Students should articulate that the change in water level is correlated with the volume of the object placed in the water, and independent of the object's weight.

\section{Designing investigations, collecting, representing, and analyzing data (cluster 2)}

LE/LP 5. Design an experiment to establish that a very small piece of material has weight (when the scale available does not detect that weight) and to measure its weight. Justify the method being used.

Criteria:

o Students should choose to measure the weight of a set of equal- sized small pieces (these must be big enough to be counted).

o Student justifications should include the idea that weight is proportional to amount of material (i.e., the weight of the set is the sum of the weights of the individual pieces); moreover, if you add enough small pieces together, they should add up to something detectable by the unaided senses, even if the small pieces are very light.

o In designing the experiment, students should note that heft is not a reliable-measure of weight and that different scales have different sensitivities.

LE/LP 6 Design an experiment to establish that a very small piece of material has volume, no matter how tiny it is; and to measure its volume. Justify the 


\section{Structure, Properties, and Transformation of Matter: Level 2}

White background-structure \& properties of matter; Grey background - transformation of matter

method being used.

Criteria:

o Students should choose to measure the volume of a set of equal size small pieces using water displacement.

o Student justifications should include the idea that volume is proportional to amount of material (an extensive property).

o Student justifications should also include the idea that if you add enough small pieces together they will take the same volume as a large sample, even if each individual piece looks like almost nothing.

LE/LP 7: Collect and represent data that will allow one to determine which objects (among regularly shaped objects such as cubes, or cylinders varying only in height) could be made of the same material when no visual information is available about the materials.

Criteria:

o Students should invoke heaviness for size as the relevant property of materials to consider.

o Students should measure the volumes and the weights of the objects and use their representation of the data to infer differences or similarities in material identity.

\section{Constructing scientific arguments (cluster 3)}

LE/LP 8. Use a model to challenge the claim that two small pieces of different materials weigh the same because they feel the same.

Criteria:

o Students should say that heft is not a reliable measure of weight. They should say that objects made of some materials can be heavier for their size than objects made of other materials and that this holds for all sizes.

o Students should invoke the compositional model of matter to link the weights of two large chunks to the weight of the two small pieces.

LE/LP 9. Critique a claim that liquid materials and solid materials are different materials.

Criteria:

o Students should point out that there are many different materials, and that one particular material can usually transition from one state to another while it remains the same material

Learning Performances informed by work of Wiser and colleagues with elementary students (TERC, 2011; Wiser, Smith et al., In Press)

Level 2 Students' Explanation or Explanatory Model(physical transformations) 


\section{Structure, Properties, and Transformation of Matter: Level 2 \\ White background-structure \& properties of matter; Grey background - transformation of matter}

What is taking place when matter changes?

Focus Question 1: What happens to matter when it is physically divided into very small pieces (grinding)

When a "chunk" of solid material is ground or crushed into a powder or dust, the powder may look different, but it is still the same material because it is

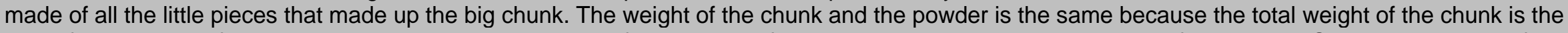
sum of the weights of all the little pieces. Likewise the sum of the volume of all the little pieces equals the volume of the chunk. One can also think of samples of a liquid as made of tiny drops of that liquid; each drop has weight and volume.

Focus Question 2: What happens to matter when it dissolves?

When a solid or powder dissolves in a liquid, it may become invisible to the naked eye but it continues to exist and the liquid and the dissolved material have the same total weight as before they were mixed together.

Focus Question 3: What happens to matter when it melts and freezes?

When a chunk of solid material melts or when a sample of liquid freezes, it is still the same material and weight does not change.

\section{What has changed in students' thinking from the previous level?}

o Prior to this level, material identity is conserved only when an object is cut into pieces big enough to keep all the perceptual properties of the object. Students at this level move beyond perceptual evidence and develop an ability to think about material samples as constituted of very, very tiny pieces (i.e. detectable only with sensitive scales or light microscopes) that can be generated by grinding a solid object. This allows students to understand that material identity is conserved when solid chunks are broken down into very small size pieces that the unaided eye cannot detect.

o Now students can integrate their ideas of conservation of material identity, conservation of amount of material and weight constancy during transformations. These integrated ideas can be used to explain phenomena such as dissolving and mixing, which could not be well explained before.

o Before, the state of matter was an inherent property of materials. Now students realize that material identity and being liquid or solid are two different things.

\section{Student Ideas \& Boundaries (physical transformations)}

Prior Ideas, Naive Conceptions, Challenges

Students at the previous level tend to hold the beliefs that solids and liquids are very different kinds of things, and that being solid or liquid is an inherent

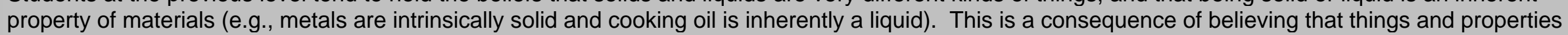
are defined by appearance. When materials change state, salient perceptual changes occur, leading to the idea that the material has changed. For example, many students say wax becomes water when it melts (Stavy, 1991). Similarly when a material dissolves, students who trust their senses say it has disappeared (Piaget \& Inhelder, 1974). These ideas constitute obstacles to learning that material identity is conserved across melting and freezing. 


\section{Structure, Properties, and Transformation of Matter: Level 2 \\ White background-structure \& properties of matter; Grey background - transformation of matter}

Students also have difficulty applying the idea of temperature to change of state (Driver, Guesne et al. 1985). Therefore they need to integrate the phenomena of melting and freezing, the notion of melting point, the conservation of material identity during melting and freezing.

\section{Useful or Productive Ideas(levers or stepping stones)}

The exploration of freezing and melting should start with the familiar case of water but should include other materials as well. If students explore freezing and melting only with water, they are likely to infer (implicitly) that water is an exception, and that other materials change identity when they melt or freeze. The idea can then be built up by exploring freezing and melting of other materials, starting with materials that maintain perceptual properties such as color and smell when they melt (e.g., chocolate). The idea that metals at room temperature are "frozen" may be provocative enough to promote thinking of materials as existing in liquid and solid state depending on temperature, rather than as an inherent property of materials. Differentiation of material identity and state of matter will serve as a stepping stone for differentiating state and material kind more generally in the next level (Grades 5-6) to include gases and in changes of state involving gases (evaporation, and condensation) (Terc 2011).

\section{Boundaries}

Evaporation and condensation should not be explored before the material nature of gases has been established. They are topics for the next level (Grades 5-6).

\section{Rationale and Notes}

o The microscopic compositional model supports an understanding of the dissolving process. If students can imagine a solid as breaking into very small pieces that have weight, they will be more likely to understand why, even if the solid becomes invisible, the solute continues to exist and the amount and weight of material does not change. The model also helps students understand that the amount, identity, and weight of a sample of material stay the same when it melts or freezes.

- Students are continuing to deepen their understanding of material, in particular, they realize that some materials exist in solid or liquid state, depending on temperature, and can also exist in a granular (or aggregate) state. This is a lever to the more general idea, developed at the next level, that matter can exist in a liquid, solid and gas form. In the next level (Grades 5-6), weight and volume will be used as evidence for the presence of matter that cannot be seen or felt, and for the conservation of amount of matter during phase changes. Therefore, robust concepts of weight and volume need to be constructed in this level.

o Measurement continues to be an integral part of constructing scientific concepts in this level, such as weight, volume, and melting point.

- Developing the idea that some of the properties most relevant to identifying materials cannot be assessed perceptually is part of developing a more scientific concept of material which helps students establish the conservation of material identity during grinding, melting and freezing, and is a stepping stone to conservation of material identity across phase change at the next level.

Notes on how students demonstrate and apply their knowledge (as reflected in Learning Performances) 


\section{Structure, Properties, and Transformation of Matter: Level 2 White background-structure \& properties of matter; Grey background - transformation of matter}

o Phenomena that students investigate or explain include grinding, melting, freezing and dissolving. [Before, chopping or other pieces at scales that are visible to the eye were explored].

o Phenomena that students predict and explain involve a wider range of materials.

o In explaining and constructing arguments for differences or similarities among different objects students should be able to consider material identity, weight, and amount of material and volume as central properties of solid and liquid samples, and should know that weight and volume are proportional to amount of material.

In designing investigations students should be able to draw upon quantitative reasoning and conservation of material identity to justify designs and take into consideration some non-perceptual intrinsic properties of materials (e.g., boiling point).

o In designing investigations and constructing arguments students should more consistently consider scales to examine weight [instead of using heft] because they should realize scales are more sensitive and accurate.

\section{Sample Learning Experiences \& Learning Performances (LE/LP) (physical transformations)}

Explaining, predicting, developing explanatory models (cluster 1)

LE/LP 1. Predict and justify whether a solid material changes identity when it is ground.

Criteria:

o Students should predict that material identity is conserved.

o Students should explain that grinding does not change material identity because the solid chunk was composed of the grains. They should predict that the ground material keeps some of the properties of the solid materials, such as smell, melting point, and flammability.

LE/LP 2. Predict and justify whether a solid material keeps its identity when melted.

Criteria:

o Students should justify material identity conservation by invoking material properties such as smell, color, solubility in water, and "greasiness".

LE/LP 3. Predict whether a mixture of powder and water will weigh the same, less, or more than the sum of the weights of the liquid and the solid before they were mixed, and explain why.

Criteria:

o Students use the idea that solid materials can break into tiny pieces when placed in water; the pieces are too small to be visible with the naked eye. They should predict that weight is conserved because the amounts of powder and water are conserved.

Designing investigations, collecting, representing, and analyzing data (cluster 2) 


\section{Structure, Properties, and Transformation of Matter: Level 2}

White background-structure \& properties of matter; Grey background - transformation of matter

LE/LP 4. Design an experiment to test which of several similar-looking powders resulted from grinding a given solid sample.

Criteria:

o Students measure and compare the weight of the solid sample and each powder sample. If several powder samples match the solid sample in weight, the student measures melting point of the solid material and the powders. 


\section{Structure, Properties, and Transformation of Matter: Level 3 \\ White background-structure \& properties of matter; Grey background - transformation of matter}

\section{LEVEL 3 (Grades 5-6): Particle Model of Matter}

\section{Overview}

Students hold a particle model of matter in which all matter (be it in a solid, liquid, or gas form) is comprised of tiny particles that are in constant motion. Students now have a description of, and explanation for, gases, and a basis for investigating them, and phase changes involving them.

\section{Level 3 Student Explanation or Explanatory Model}

What is matter made of and how does its composition relate to its properties?

\section{Focus Question 1: What is matter, and how are some of its properties determined?}

Solid, liquids, and gases consist of extremely tiny particles that cannot be seen with either the unaided eye or with a light microscope. There is empty space between particles. Particles in gases are much further apart than particles in solids and liquids. Because the tiny particles in gases are far apart they cannot be seen. Each particle weighs a tiny amount, so gases have weight. Since gases have weight and occupy space, they are matter, like solids and liquids. Gas particles move in straight lines until they hit other particles or the walls of the container. For each particular substance, particles in the liquid phase move more slowly than particles in the gas phase for that same substance, and particles in the solid phase move still more slowly (except at the point the phase is changing such as at the boiling or melting points). Particles in a solid move back and forth around fixed positions without colliding with each other.

Different substances are made of different "particles".

Focus Question 2: What causes materials to expand or contract?

When a solid, liquid, or gas is heated, the average speed of the particles increases and there is a slight increase in the average distance between the particles. This change in speed and spacing upon heating results in an increase in volume. Upon cooling, materials contract. Contraction is possible because there is empty space between particles, so there is room to compress into a smaller volume. As learned in Level 2, with enough heating, a phase change will occur.

\section{What has changed in students' thinking from the previous level?}

o Students move from a microscopic compositional model to a particle model of matter (reflecting a major shift in thinking). In the previous grade band, they could envision materials as made of arbitrarily small pieces but those pieces did not pre-exist in materials, they were created by physically or mentally dividing a sample of material. The particulate model is a stepping stone preparing students for the atomic-molecular model presented in level 4 (Grades 7-8).

o The particulate model allows students to make sense of gases being matter, as gases have weight and occupy space. Students' concept of matter at the macroscopic level increases in sophistication to include types of matter that are not detectable by touch or sight.

o The particulate model provides a powerful explanation for the difference between materials, including density, and consolidates the differentiation 


\section{Structure, Properties, and Transformation of Matter: Level 3 White background-structure \& properties of matter; Grey background - transformation of matter}

between material kind and state of matter, in other words students fully recognize that a given material can exist in different states.

o The concept of density can be further explored at this grade; students move from objects being heavy for their size to materials being more or less dense. Density is a function of the particles and the space between particles, and varies from substance to substance.

o At the previous grade band students developed an awareness that solids and liquids share some similarities (e.g., they have weight and volume), but that in some ways they behave differently. At this level, students learn that solids and liquids are composed of particles (as are gases). The properties of liquids, solids, and gases are determined by the identity and behavior of these particles.

o Phase changes involving gases (evaporation, condensation) are added to students thinking.

\section{Student Idea \& Boundaries}

\section{Prior Ideas, Naive Conceptions, Challenges}

The ideas that gases have mass and volume and therefore are matter is difficult for students (Driver 1994).

Students have difficulty differentiating weight and density (Driver 1994).

It is difficult for students to conceive of particles moving in a vacuum; that there is 'nothing' between the particles. It is common for them to think that the space between particles of a gas contains air or other particles (Novick \& Nussbaum, 1978). Students find it equally challenging to visualize that these particles move. (Novick and Nussbaum 1978; Lee, Eichinger et al. 1993; Johnson 1998).

Students also have a difficult time understanding very small sizes, and distinguishing among objects that are too small to see with the unaided eye (Delgado 2009).

Most middle school students' knowledge of matter is very fragmented (Nakhleh, Samarapungavan \& Saglam, 2005), due to difficulties "assimilating the microscopic level scientific knowledge acquired through formal instruction into students' initial macroscopic knowledge frameworks."

\section{Useful or Productive Ideas (levers or stepping stones)}

To help students realize that there are spaces between particles, students can observe that gases can be added to another gas, expand to fill a container, and can be compressed and expanded. These observations can build to an understanding of the empty space between particles in the liquid and solid phases.

\section{Boundaries}

Atoms and molecules are not the focus at this level. Therefore, students are not ready for chemical reactions or chemical properties. 


\section{Structure, Properties, and Transformation of Matter: Level 3 White background-structure \& properties of matter; Grey background - transformation of matter}

\section{Rationale and Notes}

o The particle model is a very important intermediate model of matter. It is not scientifically complete, since it does not include a notion of atoms and molecules, but it is critical for getting students to think about matter at very small scales and to account for gases, thermal expansion and contraction, and density. However this model does not account well for chemical reactions and therefore its applications are limited to physical properties of substances.

o The particle model is a substantial advance over the microscopic compositional model, and students will need to explore and use the particle model extensively. In particular, the notion that the particles move at different speeds for different states, for a given substance, is difficult and may develop later in the grade band.

o Expansion and contraction are helpful phenomena for students to explore because they are best explained by a particle model of matter. Thus when students are critiquing or debating different models, it would be evident that the particle model best explains the phenomena (Merritt 2010).

\section{Notes on how students demonstrate and apply their knowledge (as reflected in Learning Performances)}

- Phenomena that students explain, investigate, and argue about now include gases [which were not considered before]; therefore students can explore and explain boiling, evaporation, and expansion of gases.

- Phenomena to explain or investigate are more complex such as dissolving or expansion of gases upon heating, and students can use the particle model to account for these.

- In explaining and constructing arguments about the properties of matter, students use and apply the particle model, but explanations are essentially descriptive and don't identify deeper mechanisms to account for why particles behave as they do.

- In designing investigations and constructing arguments, students can now draw upon a wider range of intrinsic properties [e.g. density and boiling point] to distinguish and identify different materials. 


\section{Structure, Properties, and Transformation of Matter: Level 3 \\ White background-structure \& properties of matter; Grey background - transformation of matter \\ Sample Learning Experience \& Learning Performances (LE/LP)}

Explaining, predicting, developing explanatory models (cluster 1 )

LE/LP 1. Students provide an evidence-based explanation for why gases are matter, despite seeming different from solids and liquids. Criteria:

o Students' explanation should include evidence that gases have mass and take up space (collected from empirical investigations).

LE/LP 2. Draw models to explain what makes different solid materials different from each other.

Criteria:

o Students' models should include the idea that different materials are made of different particles. A common error would be to represent particles without space between them. Another common error would be to represent particles embedded in a substrate.

LE/LP 3. Students construct models that represent various phases (states) for one particular substance (e.g., water). Criteria:

o Students' models should show that in a solid state the particles vibrate back and forth around fixed positions without colliding with each other.

o Students' models should show that when a substance is in its liquid state the particles are not in fixed position, but instead can collide and slide past each other.

o Students' models should show that when a substance is in the gaseous state the particles are very far apart on average and that they move in all directions, occasionally colliding with one another.

\section{Designing investigations, collecting, representing, and analyzing data (cluster 2)}

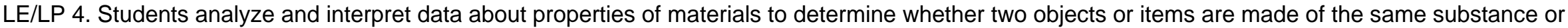
different substances, and to identify each substance.

Criteria:

o Given a set of properties, students should select which are intrinsic properties (e.g., characteristic of a substance). Students should compare the intrinsic properties of items to determine if the materials are the same or different and then use the intrinsic properties to determine what substances the objects are made of.

LE/LP 5. Students design an investigation to determine whether two objects are made of the same or different substances. Criteria:

o Students' designs should clearly specify which properties will be identified and how [again students at this level should rely on a wider variety of intrinsic properties].

o Students' plans should also more advanced-e.g. they make a prediction, identify variables, control variables, and communicate and defend their scientific procedures.

o Students' plans should clearly specify various variables and how the data will be collected and analyzed. 


\section{Structure, Properties, and Transformation of Matter: Level 3 \\ White background-structure \& properties of matter; Grey background - transformation of matter}

Constructing Scientific Arguments (cluster 3)

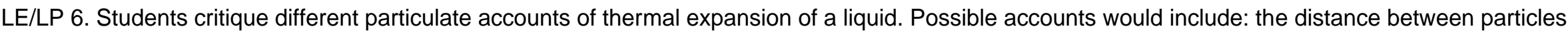
increases; particles get bigger; heat particles insert themselves between the particles of the liquid.

Criteria:

o Students should state that the volume of individual particles does not change with temperature; as heat increases, the speed of the particles increases on average and the distance between particles also increases on average.

Learning Performances informed by work of Krajcik and colleagues with middle school students (Krajcik, McNeill et al. 2008; Merritt 2010).

\section{Level 3 Student Explanation or Explanatory Model (physical transformations)}

What is taking place when matter changes?

Focus Question 1: What happens when matter changes state, in terms of particles?

When the temperature of a solid material increases, its particles move faster until the temperature reaches the melting point of the material. At the melting point, the particles start moving differently and the material becomes liquid. When temperature reaches the boiling point, particles start moving away from each other and move freely to fill the space available. The particles are the same regardless of the state of the material. The number and type of particles stays the same across phase changes, therefore weight stays the same.

Focus Question 2: What happens when matter dissolves?

When a solid sample of material dissolves in a liquid, the particles of the solid sample move in between particles of the liquid. The solid material may become invisible because its particles move further apart from each other, but not far enough apart to change into a gas state. The amount of solid material does not change because the number of its particles stays the same. The weight of the mixture is the sum of the weight of the solid sample and the liquid.

\section{What has changed in students' thinking from the previous level?}

- At this level conservation of material identity, amount of material, and weight across phase change and dissolving, can be accounted for by the particle model and therefore are much more deeply justified than before.

\section{Student Ideas \& Boundaries (physical transformations)}

Prior Ideas, Naive Conceptions, Challenges

Students often confuse melting with dissolving.

Students often misuse the particulate model in their account of physical changes by attributing macroscopic properties/behaviors to the particles. They often talk about particles melting, for example, or particles getting bigger when temperature increases (Kind, 2004). 


\section{Structure, Properties, and Transformation of Matter: Level 3 \\ White background-structure \& properties of matter; Grey background - transformation of matter}

An idea that can present some challenges for students is that the boiling point or melting point occurs at a single temperature-even though the processes of boiling and melting occurs over a period of time.

Often, students will believe that a physical transformation results in new materials being formed and that a permanent change occurs. For instance, students often think that a material disappears as a result of evaporation or boiling. They also might believe that a substance disappears when it is dissolved in another substance (Lee et al., 1998; Nakhleh \& Samarapungavan, 1999).

Useful or Productive Ideas (levers or stepping stones)

It is critical for students to realize the mass of the substance undergoing a physical transformation remains constant during the transformation (in a closed system). If students can also recognize that the total number of particles does not change across a physical transformation, this may be leverage to being able to track the type and number of atoms present during and after a physical or chemical transformation at the next level.

\section{Boundaries}

Students should not focus on chemical reactions yet.

Applying conservation of matter at this level should only be applied to physical changes because they do not necessarily require an understanding of the difference between atoms and molecules. In contrast, an understanding of the conservation of matter as it applies to chemical changes requires an atomicmolecular model of matter to keep track of the number of atoms both before and after any change (physical or chemical). Thus developing conservation of matter as a general rule that applies to all transformations should be targeted at the subsequent level.

\section{Rationale and Notes}

o At the previous level (Grades 3-4), students became familiar with melting, freezing, and dissolving. Weight constancy was used as evidence for conservation of amount of material during melting and freezing. Conservation of material identity during melting and freezing was explored systematically, but in this level, the particulate model provides a much stronger explanation. The particulate model also captures the difference between melting and dissolving in a compelling way. The particulate model account of physical transformations paves the way for an atomicmolecular account of chemical transformations.

- Research suggests students must have an atomic-molecular model to begin to understand chemical reactions (Harrison and Treagust 2000; Johnson, 2000) and that they must have at least a particle model to understand changes of state and other physical changes such as dissolving (Johnson, 1998; Papageorgious and Johnson, 2005), therefore it is at the next level that students begin to distinguish physical changes from chemical changes. However, research suggests that this distinction is difficult for students (Ahtee and Varjola 1998; Johnson 2000; Krajcik, McNeill et al. 2008). 


\section{Structure, Properties, and Transformation of Matter: Level 3}

White background-structure \& properties of matter; Grey background - transformation of matter Notes on how students demonstrate and apply their knowledge (as reflected in Learning Performances)

- Phenomena that students explain, investigate, and argue about now include gases; therefore students can also explain and explore boiling, evaporation, and expansion of gases.

- Phenomena that students explain or investigate, such as dissolving or expansion of gases upon heating, are more complex, since students can use the particle model to account for them.

- In explaining and constructing arguments about the transformation of matter, students use and apply the particle model, but explanations are essentially descriptive and lack deeper mechanisms to explain why particles behave as they do.

- In designing investigations and constructing arguments, students can now draw upon a wider range of intrinsic properties [e.g. density and boiling point] to distinguish and identify different materials.

\section{Sample Learning Experiences \& Learning Performances (LE/LP) (physical transformations)}

\section{Explaining, predicting, developing explanatory models (cluster 1 )}

LE/LP1. Draw a model that explains the dissolution process, e.g., why dissolved substances can become invisible; why a drop of ink disperses faster in hot than cold water.

Criteria:

o Using the particulate model, students should draw two different kinds of particles mixing with each other.

o Students should explain that particles are so tiny that they cannot be seen individually even with a light microscope. In some cases, the solute is still visible but appears continuous; in other cases, the particles of solute are far enough apart that they become invisible.

o For phenomena involving different temperatures, students should explain that particles move faster and move over greater distances when temperature increases.

Designing investigations, collecting, representing, and analyzing data (cluster 2)

LE/LP2. Design an experiment to test whether weight changes when a solid sample melts. Use a particulate model to explain the results of the experiments.

Criteria:

o Students should weigh the sample before and after melting.

o Students should explain that weight does not change because the number of particles stays the same; when a solid melts, its particles move differently but do not increase or decrease in number. Each particle weighs a tiny bit. Since the particles continue to exist and are individually unchanged, their weight stays the same. The weight of the sample is the sum of the weight of the individual particles, and therefore stays constant. [Students should not talk about particles themselves becoming liquid or changing in size].

LE/LP3. Design an experiment to refute the claim that the water appearing on a cold container filled with water when it is placed in a warm environment comes from inside the container.

Criteria: 


\section{Structure, Properties, and Transformation of Matter: Level 3}

White background-structure \& properties of matter; Grey background - transformation of matter

o Students should refer to the phenomenon as condensation and explain it. [Experiments could consist of dyeing the water inside the container and showing that the condensed water is clear; bringing an empty cold container into a warm environment, showing that condensation still takes place; or weighing the water inside the container to demonstrate its amount has not changed during condensation].

LE/LP4: Design an investigation to demonstrate that amount of matter is conserved when a liquid evaporates.

Criteria:

o Students should design a closed system, placed in a warm environment or in proximity to a source of heat. They should measure the level of liquid at repeated intervals and weigh the system to establish that weight does not change.

o Students should reason that weight is correlated with amount of matter, and therefore, the liquid is not disappearing but is still in the system, although in invisible form [this requires a particle model to argue effectively].

Constructing scientific arguments (cluster 3)

LE/LP5. Use particle models to refute the claim that melting, mixing, and dissolving are the same process.

Criteria:

o Students' critiques should account for melting in terms of movement of particles and represent one kind of particle in both states. Their critiques should also account for dissolving and mixing using two kinds of particles.

Learning Performances informed by work of Krajcik and colleagues with middle school students (Krajcik, McNeill et al. 2008; Merritt 2010) 


\section{Structure, Properties, and Transformation of Matter: Level 4}

White background-structure \& properties of matter; Grey background - transformation of matter

\section{LEVEL 4 (Grades 7-9): Atomic-molecular Model of Matter}

\section{Overview}

Students hold an atomic-molecular model of matter in which the particles are conceived of as being either atoms (or collections of atoms forming molecules) and the molecules are in constant motion. Attractive forces and energy influence how the molecules interact and behave with each other. Students can now adequately explain (descriptively) chemical changes along with new properties that require an understanding of the interactions between molecules such as differences in the melting points of different substances.

\section{Level 4 Student Explanation or Explanatory Models}

What is matter made of and how does its composition relate to its properties?

\section{Focus Question 1: What is matter, and how are its properties determined by the atoms and molecules that compose it?}

As in the previous level, matter is composed of tiny particles that move about, however the particles can be either atoms or combinations of atoms held tightly together (molecules).

\section{Focus Question 2: How are some of its properties determined?}

According to the atomic-molecular theory, different substances have different chemical properties because they have different molecular composition and/or structures (arrangements of atoms). One inherent property of a substance is reactivity, which reflects the tendency for some types of atoms to interact and aggregate with other atoms (or molecules) of the same or another type (one useful example might be oxygen's ability to react with hydrocarbons). Some substances have molecules made of the same type(s) and same number of each type of atom; but, the arrangement of the atoms that make up the molecules is different. In this case, you have two different substances because they have different properties stemming from the different arrangement of atoms. Related to this idea, the concepts of pure substances and mixtures can now be more adequately explained: a pure substance is made of the same type of atom or molecule throughout, while a mixture contains more than one type of atom or molecule throughout.

\section{Focus Question 3: What are its states?}

When a substance is in its liquid state the attractive force between the molecules is weaker compared to when the same substance is in the solid state and therefore the molecules are not in fixed positions. Instead, in the liquid state, the particles can collide and slide past each other. Higher temperatures of a substance in the liquid state mean the molecules move more quickly and have more kinetic energy. When the same substance is in the gas state the attraction between its molecules is negligible, and can be ignored. In the gaseous state the molecules of the substance are moving faster than in the liquid state and have more kinetic energy. The molecules on average are very far apart in gases, compared to the distance between molecules in the solid or liquid state; molecules in a gas move in all directions, occasionally colliding with one another. Higher temperatures mean they move with greater speed and greater kinetic energy.

\section{Focus Question 4: How are some of its properties determined?}

Solids or liquids expand when heated and contract when cooled. In terms of forces, this happens because as the temperature rises the molecules move (or vibrate) with greater speeds. When this happens, the molecules tend to move further away from each other, weakening the strength of the attractive forces between the particles. As the temperature decreases, the particles move with lower speeds. When this happens the molecules tend to move closer 


\section{Structure, Properties, and Transformation of Matter: Level 4}

White background-structure \& properties of matter; Grey background - transformation of matter

together and the strength of the attractive forces between them increases.

In terms of energy, when a solid or liquid material is heated (but not during melting or boiling), heat energy is transferred into the material, and this causes two things to happen. First, the average kinetic energy of the molecules increases, resulting in a rise in temperature. Second, the potential energy of the molecules increases as the molecules move a little further apart, and this results in the whole solid or liquid material expanding. [Students would need to recognize that as the distance between particles that attract each other increases; the potential energy of the system of particles increases- this would be a connection to the energy strand in the NRC document]. When a solid or liquid is cooled (but not during freezing or condensation), heat energy is transferred from the system and this causes two things to happen. First, the average kinetic energy of the particles decreases, resulting in a lowering of temperature. Second, the potential energy of the particles decreases as the molecules move a little closer together, resulting in the whole solid or liquid material contracting.

A gas exerts pressure on any surface it is in contact with because the molecules that are in constant motion push on the walls of the container when they collide with it. When the molecules move faster, they push harder on the walls and the pressure increases. Because the molecules move faster, they also collide more frequently with the walls of the container. This increase in the frequency of collision also accounts for increased pressure. The opposite happens when the temperature decreases.

The density of a substance is how much mass there is for each unit volume of the substance. This value does not depend on how much of a substance you have; thus it is known as a characteristic (or intrinsic) property and can help distinguish one substance from another. One substance has a higher density than another substance because its molecules have greater mass and/or because the molecules are on average closer together.

\section{What has changed in students' thinking from the previous level?}

o Previously, any internal structure of particles was ignored. Now students focus specifically on the internal structure of the particles. Particles are now considered to be composed of one or more atoms, arranged in units called molecules. It is the composition, arrangement and interactions between these atoms and molecules that give substances their "chemical" properties. (Note: While technically some substances may be composed of atoms not forming molecules, most substances that students would explore and investigate would be substances composed of molecules and therefore in their explanations students would refer to the particle as "molecules").

o The model of how particles behave (now understood to be atoms or molecules) includes the idea of attractive forces and the energy associated with the particles. This moves students from simply describing how matter behaves in different states to providing an understanding of (description of) the mechanisms that cause matter to behave differently in different states. Specifically, the model at level 3 can only describe how the particles' spacing is different, not why. The model here also allows students to explain more intrinsic properties of substances. For example, the ideas of energy and forces introduced here can be used to help explain why some liquids bead up (surface tension) or have different melting or boiling points.

o Students are essentially elaborating and extending upon the particle model and this would occur over the course of several years. Thus, the models and explanations outlined above might not fully develop until late middle school or early high school. Students would likely not simultaneously re-conceptualize the particles as molecules and add ideas of attractions and energy to their existing model of matter. 


\section{Structure, Properties, and Transformation of Matter: Level 4 White background-structure \& properties of matter; Grey background - transformation of matter}

\section{Student Ideas \& Boundaries}

Prior Ideas, Naive Conceptions, Challenges

Students can develop many unproductive ideas about atoms and molecules (e.g.,"Raisins-in-the-pudding" model, with atoms embedded in material; "atoms as little stacked cubes"1; or the idea that atoms are too small to have weight or occupy space) (Wiser, personal communication).

Often students assign macroscopic properties of substances to the atoms or molecules that compose the substance (Ben-Zvi, Eylon \& Silberstein, 1986;

Nakhleh, Samarapungavan \& Saglam, 2005). For instance, they will think of a particle of copper having a copper color.

There is abundant data that students have a difficult time distinguishing atoms from molecules and relating relative size of atoms to microscopic structures like cells (Stevens, Delgado et al. 2010). Students often confuse molecules and cells (Arnold 1983).

Many students find it challenging to distinguish between an atom, an element, molecule, a compound, substances and a mixture (Devetaka and Glažara, 2010).

Even at the college level, students do not have understandings of the forces that exist between atoms to form molecules (Stevens, Delgado, and Krajcik, 2010).

\section{Useful or Productive Ideas (stepping stones)}

It is critical that students are able to distinguish among atoms, elements, molecules, substances and mixtures.

The exploration of the composition of "particles" in terms of atoms paves the way for thinking of atomic structure in future levels.

The idea of forces between molecules is a precursor to thinking about reactivity in high school chemistry classes.

\section{Boundaries}

Students do not consider the subatomic structures of atoms such as neutrons, protons, or electrons in their conceptions of matter at this level.

\section{Rationale and Notes}

o Although the particle theory described at the previous level can be used to descriptively explain physical changes (e.g., changes of state), and can account for conservation of mass during physical changes, it cannot explain chemical changes nor conservation of mass during chemical changes. Therefore, at this level, when students begin considering chemical changes, their model of matter must evolve to include atoms and molecules, that is, the substructure of the "particles." Thinking in terms of atoms and molecules will be necessary in order to understand what happens during chemical changes and why mass is still conserved, since student understanding is no longer limited to the idea that during chemical changes the total number of "particles" remains constant (e.g., in the electrolysis of water, two molecules of water become two molecules of hydrogen and one molecule of oxygen and during this reaction the number of each type of atom remains does not change before and after the reaction).

\footnotetext{
${ }^{1}$ Probably due to hearing "If you keep cutting a piece of matter into smaller and smaller pieces, you end up with atoms."
} 


\section{Structure, Properties, and Transformation of Matter: Level 4}

White background-structure \& properties of matter; Grey background - transformation of matter

Notes on how students demonstrate and apply their knowledge (as reflected in Learning Performances)

- Phenomena that students can explain, investigate, and argue about include a wider range of intrinsic properties; ones that require the idea of attractive forces or energy to explain [e.g. pressure exerted by gases].

- In explaining or constructing arguments, students can use and apply ideas of atoms and molecules [as opposed to the general notion of a small particle applied before]; for instance students account for conservation of matter during transformations by paying attention to the numbers and types of atoms [whereas before students could only think of total number of particles or conceive of conservation of macroscopic weight].

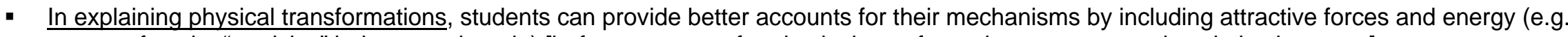
reasons for why "particles" behave as they do) [before, accounts for physical transformations were more descriptive in nature].

- In designing investigations students can take into account an even greater variety of intrinsic properties of materials, including reactivity with specific substances.

- In critiquing others' models or explanations use the idea of attractive forces, which is a clearer mechanism for the behavior of molecules. Consequently, students can fault other students' explanations for not including such ideas. [In this way students' arguments rely on more sophisticated models]

\section{Sample Learning Experiences \& Learning Performances (LE/LP)}

Level 4: Structure \& Properties

Explaining, predicting, developing explanatory models (cluster 1)

LE/LP1. Students provide an explanation for what makes one substance different from another substance.

Criteria:

o Students' explanations should include the idea that the different substances are composed of different atoms and/or molecules of different types, numbers and/or arrangement of atoms.

LE/LP 2. Students construct models to show the difference between a substance and a mixture.

Criteria:

o Students' models should show that a substance is made of the same type of atom or molecule throughout and that a mixture contains more than one type of atom or molecule throughout.

LE/LP 3. Students construct models that show why the pressure a gas exerts changes with a difference in temperature. Criteria:

o Students' models should show that gas molecules are in constant motion and push on the walls of the container when they collide with it. When the molecules change temperature, their average motion changes. When the temperature increases the molecules move faster, 


\section{Structure, Properties, and Transformation of Matter: Level 4}

White background-structure \& properties of matter; Grey background - transformation of matter

they push harder on the walls, and the pressure increases. Because the molecules move faster, they also collide more frequently with the walls of the container. This increase in the frequency of collision also accounts for increased pressure. The opposite happens when the temperature decreases.

Designing investigations, collecting, representing, and analyzing data (cluster 2)

LE/LP 4. Students design and justify an investigation to determine whether two objects are made of the same or different substances. They discuss how the substances are the same or different in atomic-molecular terms and upon draw intrinsic properties of substances. Students make a prediction, identify variables, control variables, and justify their observational and experimental procedures.

Criteria:

o Students' designs should clearly specify which properties will be identified and how.

o Students should be able to draw upon an even wider variety of intrinsic physical properties, this may include boiling point and density, but also chemical properties like reactivity, since at this level students should be able to explain chemical reactions.

o Students' plans should clearly specify variables and how the data will be collected and analyzed. Students should also communicate their design in a clear and logical manner, and defend their design against questions about threats to the validity of their expected conclusions. Students' explanations use the atomic-molecular model to discuss why the materials are the same or different.

\section{Constructing scientific arguments (cluster 3)}

LE/LP 5. Students critique explanations for why a liquid expands upon heating. Criteria:

o Students' critiques should explicitly point out that an explanation needs to include the idea that molecules in the higher temperature liquid are farther apart than in the lower temperature liquid.

o Students' critiques should make reference to the attractive forces between molecules and the speed of the molecules. In communicating their critiques to others, a student should state that more appropriate explanations include statements that (1) the particles are close to each other because of the attractive forces between them; and (2) as the temperature increases the molecules move with greater speeds, allowing them to move further away from nearby molecules where the attractive forces are weaker.

o Students should discuss difficulties with explanations that attribute changes in volume to the molecules rather than their spacing.

Level 4 Student Explanation or Explanatory Model (physical transformations):

What is taking place when matter changes? 


\section{Structure, Properties, and Transformation of Matter: Level 4 White background-structure \& properties of matter; Grey background - transformation of matter}

Focus Question 1: What happens when matter changes states, in terms of atoms and molecules?

When a substance undergoes a physical change (like a change in state or an increase in volume as the temperature increases), the spacing of its molecules, the strength of the interactions between the molecules, and the kinetic energy and potential energy of the system all change. The molecules do not change, however. The total number of molecules (and thus, atoms) after the change is the same as the total number before the change. Since each molecule has the same mass, this explains why the mass of a substance, composed of atoms or molecules, does not change during a physical change.

Focus Question 2: What happens during evaporation and condensation, melting, and freezing?

At any given temperature molecules of a substance have a wide range of values of kinetic energy. Therefore, evaporation can occur at temperatures well below the boiling point because there are some molecules at the surface of liquids with sufficient kinetic energy to escape the attractive forces of neighboring molecules and escape into the gas state. Also, condensation can occur over a range of temperatures because there are some molecules in the gas phase with kinetic energies so low that they can't overcome the attractive forces from neighboring molecules in the liquid, and they too become molecules in the liquid state.

Each substance has a melting point because at a certain temperature the average kinetic energy of the molecules within a solid is sufficient to weaken the strength of the attractive forces between molecules, allowing the molecules to move past each other rather than remaining in fixed positions. Each substance has a unique melting point because the arrangement and strength of attraction between molecules is different for different substances.

Each substance has a boiling point because at a certain temperature the average kinetic energy of the molecules within a liquid is sufficient to overcome the attractive forces of nearby molecules, allowing the molecules to separate far from each other and become particles in the gas state. Each pure substance has a unique boiling point because the arrangement and strength of attraction between molecules is different for different substances

During both melting and boiling all the heat energy transferred into the material causes just the potential energy to increase; the average kinetic energy remains constant. This increase in potential energy reduces the attractive forces among the molecules. Thus, the temperature remains constant during melting or boiling. During melting, as the potential energy increases, the molecules break away from their fixed positions and begin to move past each other (and the material becomes a liquid). For some materials more heat energy is needed for the molecules to break the force of attraction among the particles than is needed for other materials; hence they have a higher melting point. During boiling, as the potential energy increases the molecules move very far from each other and travel in all directions (and the material becomes a gas). For some materials the heat energy needed for this to happen is greater than for other materials.

What has changed in students' thinking from the previous level? 


\section{Structure, Properties, and Transformation of Matter: Level 4 White background-structure \& properties of matter; Grey background - transformation of matter}

o At the previous level students knew that mass (weight) was conserved during a physical change, but now they can use the atomic-molecular theory to explain why by keeping track of the type and number of atoms.

o At the previous level students could only apply conservation of matter to physical transformations, but now that they have an atomic-molecular model they can apply conservation of matter to both physical and chemical transformations.

o In general, students move from a descriptive explanation of physical changes to an explanation with a clearer mechanism for physical change. For example, at the previous level, students knew that each pure substance had unique melting and boiling points. Now they can apply the atomicmolecular theory and either force or energy ideas to describe the mechanisms that explain melting and boiling points, and perhaps also why these are unique for each substance.

\section{Student Ideas \& Boundaries (physical transformations)}

Prior Ideas, Naive Conceptions, Challenges

Students will struggle to apply conservation of matter to the liquid-gas transition (Mohan et al., 2009), because students often think that gases are weightless. Therefore they may need multiple experiences analyzing and explaining this phenomenon in the context of different substances.

Students may think that the particles of the substance have the same properties of the original substance - as they conceptualized in Level 3's particle model - rather than explaining the macroscopic properties of the substance as emerging from the collective spacing, arrangement, and interaction between the molecules. For example, some students may think that during melting particles may transition from being hard (as in a solid) to soft (as in a liquid) (Freyberg and Osborn 1985; Nakhleh and Samarapungavan 1999).

Often, students will believe that a physical transformation results in new materials being formed and that a permanent change occurs. For instance, students often think that a material disappears as a result of evaporation or boiling. They also might believe that a substance disappears when it is dissolved in another substance (Freyberg and Osborne, 1985).

\section{Useful or Productive Ideas}

The notion that there are attractive forces between molecules [in a generic sense] may be leverage to understanding that there are electrostatic interactions which influence how molecules behavior during physical transformations, which is found in students' models at the next level.

\section{Boundaries:}

Students should not focus on intra-molecular interactions yet, because they do not have a conception of subatomic particles yet and the charge associated with them.

\section{Rationale and Notes}




\section{Structure, Properties, and Transformation of Matter: Level 4}

White background-structure \& properties of matter; Grey background - transformation of matter

o Students' models undergo a substantial shift from Level 3 to Level 4. From conceptualizing arbitrarily small pieces that share the properties of the bulk material, students now transition to a model that explains the existence of these properties. In the atomic-molecular model, the macroscopic properties of the substance emerge from the collective spacing, arrangement, and interaction between the molecules. This is a difficult change that will take students most of the level to consolidate. For example, students need to realize that when a solid melts or a liquid boils, this occurs because the energy added to the system causes the arrangement of molecules in the system to change, not because each molecule changes properties like the bulk sample does.

o Research suggests that if students begin early middle school with a particle mode of matter and appropriate instruction, they develop an atomic molecular model of matter and account for chemical reactions as a rearrangement of atoms later in middle school (Krajcik, McNeill et al. 2008).

o Research suggests that when students consider both chemical and physical transformation they confuse them (e.g., Krajcik, McNeill \& Reiser, 2008), therefore it is important to explicitly compare and contrast these types of changes. At this level we include both types of transformation. At this level, and the next, we keep physical and chemical transformations separated for clarity.

\section{Notes on how students demonstrate and apply their knowledge (as reflected in Learning Performances)}

o Phenomena that students can explain, investigate, and argue about now include a wider range of transformations [now differences in boiling points].

- In explaining physical transformations, students can account for the mechanisms of physical transformations by including attractive forces and energy (e.g. reasons for why "particles" behave as they do) [before, accounts for physical transformation were more descriptive in nature]. Accounts of conservation of matter during physical transformation can now involve tracking numbers and type of atoms as well as molecules.

o In critiquing others' models or explanations students can include both descriptions of, and mechanisms for, the behavior of molecules ("particles") [in this way argumentation is also more sophisticated because it can use more sophisticated models]

\section{Sample Learning Experiences \& Learning Performances (LE/LP) (physical transformations)}

Explaining, predicting, developing explanatory models (cluster 1)

LP1. Students use a particle model of matter and force ideas to explain why a substance can exist in various phases. Criteria:

o Students explanations should include the idea that in the solid state the molecules are strongly attracted to each other, they vibrate back and forth around fixed positions without colliding with each other, and at higher temperatures they vibrate more quickly.

- Students should note that when a substance is in its liquid state the molecules are attracted with a weaker strength compared to when the substance is in the solid state and therefore are not in fixed positions, but instead can collide with and slide past each other.

o Students should note that when a substance is in its gaseous state the attraction between its molecules is negligible. On average, the 


\section{Structure, Properties, and Transformation of Matter: Level 4 \\ White background-structure \& properties of matter; Grey background - transformation of matter}

molecules in a gaseous state are very far apart compared to when the substance is in its solid or liquid state and they move in all directions, occasionally colliding with one another.

Designing investigations, collecting, representing, and analyzing data (cluster 2)

LP 2. Given a non-harmful solid (e.g., menthol), students design an experiment that shows that the substance can exist in three states, that shows that each state can be transformed into another state, and that determines the melting and boiling point of the substance.

Criteria:

o Students' plans include a procedure to measure the melting point of the solid and the boiling point of the liquid as they heat it.

o Students' plans contain at least two trials for determining the melting and boiling points.

o Students' plans also show how they will capture the gaseous phase of the substance and how they would transform the gas to the liquid and the liquid to the solid.

LP3. Given a set of data from an unknown substance that was heated from the solid state to the gaseous state, students will analyze data to determine the melting and boiling points of the substance.

Criteria:

o Given a set of data, students will need to plot the data and identify on the graph the melting and boiling points (the plateaus in the time vs. temperature graph).

Constructing scientific arguments (cluster 3$)$

LP 4. Students critique models that explain why one liquid (e.g., water) boils at a higher temperature than another liquid (e.g., acetone). Criteria:

o Student critiques of models need to point out that at the high temperature the molecules are moving faster and that the liquid with the higher boiling point also has stronger attractive forces than the liquid with the lower boiling point.

LP5. Students, when given a model-based explanation of some transformation, but one which is scientifically inappropriate, critique and evaluate the model by explaining what is problematic about it.

Criteria:

o Students correctly identify the problematic parts of the explanation, describe why they are problematic, and offer a revised version that is scientifically accurate and may offer evidence for why that can't be true (e.g., a sample problematic explanation for thermal expansion might be that the molecules increase in size).

LP 6. Students critique explanations in terms of energy for why a liquid boils only at a certain temperature (at a given pressure).

Criteria:

o Students' critiques make explicit reference to the requirement that an explanation needs to include the idea that molecules in the gaseous (vapor) state are much further apart than the molecules when in the liquid state.

o Students' critiques should make reference to several energy ideas: (1) that as a liquid is heated and the temperature rises, the heat energy transferred into the system causes both the average kinetic energy and the potential energy to increase; (2) that as the molecules get further apart, the potential energy increases; and (3) that eventually, at a certain temperature called the boiling point, all the heat energy going into the system causes just the potential energy to increase, while the average kinetic energy remains the same. This corresponds to the molecules being very far away from each other and moving in all directions, which indicates that the substance is in the gaseous state. 


\section{Structure, Properties, and Transformation of Matter: Level 4 White background-structure \& properties of matter; Grey background - transformation of matter}

LP 7. Students design an investigation and use the data collected during the investigation to argue that during phase changes, matter is conserved.

Students explain the results using atomic-molecular theory.

Criteria:

o Students' designs should show how they will determine the total mass of both the solid/liquid or the liquid/gas combinations/states that occur during the phase changes.

- Students' explanations should make a claim that mass is conserved during phase change, their data should support this claim, and their reasoning should be that atoms and molecules only change state (the atoms do not rearrange into different molecules, and molecules are not created or destroyed during phase change).

Learning Performances informed by work of Krajcik and colleagues with middle school students (Krajcik, McNeill et al. 2008; Merritt 2010)

\section{Level 4 Student Explanation or Exploratory Model (chemical transformations):}

What is taking place when matter changes?

\section{Focus Question: What happens to matter when substances change into new substances?}

When a chemical reaction occurs the atoms of the original substance rearrange or regroup into different molecules that make up a new substance. The original and new substances have different intrinsic properties. The total number and types of atoms remain the same in a chemical reaction. This idea explains the conservation of mass.

\section{What has changed in students' thinking from the previous level?}

o Students' conceptions of the transformation of matter expand greatly at this level, because now they develop an atomic-molecular model which can better explain chemical reactions. With this model, students can now explain physical transformations (such as mixing, dissolving, melting and boiling), chemical transformations, and distinguish between them.

o Students at this level can explore a wide range of chemical reactions and should be able to realize that chemical reactions take place all around them. Examples include living systems where processes such as photosynthesis and cellular respiration can be understood as chemical reactions inside of organisms. At this level they have conceptual models to explain physical and chemical transformations.

o Students can now more effectively apply conservation of matter to transformations, since they have a notion of atoms and molecules and can keep track of the numbers and types of atoms in both physical and chemical transformations.

\section{Student Ideas \& Boundaries}

Prior Ideas, Naive Conceptions, Challenges

Understanding that atoms are conserved during a chemical reaction is critical for understanding chemical transformations. Many students, even at the high school level, find it challenging to apply the idea that atoms are conserved in a chemical reaction (Hesse and Anderson, 1992). This is particularly true when the chemical transformation involves the formation of a gas (Mohan, and Chen et al, 2009). 


\section{Structure, Properties, and Transformation of Matter: Level 4 White background-structure \& properties of matter; Grey background - transformation of matter}

Students need to know that atoms make up molecules and that the molecular composition does not change in a physical transformation, in order to distinguishing physical from chemical changes. Some students confuse physical and chemical transformations and incorrectly identify physical transformation as chemical transformations (Hesse and Anderson 1992). A classic example of this thinking is seen in the belief that burning is an example of melting.

Unless students understand that atoms only rearrange during chemical reaction, they may mistakenly think that during chemical reactions atoms can change their type while molecules keep their identity (Renström, Andersson et al. 1990; Smith, Wiser et al. 2006).

Many middle school students find it a challenge to understand that a characteristic property does not change with the amount of a substance and often mistakenly identify mass and volume as a characteristic property (Smith et al., 2006).

Students will struggle to apply conservation of matter to chemical reactions in which both liquids and gas are involved as either products or reactants (Mohan, Chen et al. 2009), because students often think that gases are weightless. Therefore, they may need multiple experiences analyzing and explaining this phenomenon in the context of different substances or perhaps investigating the total mass of the substances before and after the reaction.

Secondary students are challenged to connect representations of the microscopic world of matter as they relate to macroscopic phenomena involving matter (Treagust and Chittleborough et al., 2003).

\section{Useful or Productive Ideas (stepping stones)}

Students are able to explain chemical reactions if they understand that, during a chemical reaction, the atoms in the molecules that make up the reaction rearrange into new molecules. Students do not need to talk about bonds or the role that electrons play in bonding at this level.

Understanding what a property is and that properties change as a result of a chemical reaction is a key idea in understanding whether a chemical reaction occurs. Unless students have this understanding, they do not develop more sophisticated ideas regarding the nature of chemical reactions including the idea that atoms rearrange during chemical reactions (Renström, Andersson et al. 1990).

\section{Boundaries}

At this level, students do not need to know the structure of atoms or about electrons. At the middle school level there is no discussion about how electrons are involved in bonding.

\section{Rationale and Notes}

o Research suggests that when students consider both chemical and physical transformation they do confuse them (e.g., Krajcik, McNeill \& Reiser, 2008), therefore it is important to explicitly compare and contrast these types of changes. At this level we include both types of transformation. In this section, we keep physical and chemical transformations separated, for clarity.

Notes on how students demonstrate and apply their knowledge (as reflected in Learning Performances) 


\section{Structure, Properties, and Transformation of Matter: Level 4 White background-structure \& properties of matter; Grey background - transformation of matter}

- Phenomena that students explain, investigate, and argue about now include a wider range of transformations [now chemical transformations].

- In explaining or constructing arguments, students can use and apply ideas of atoms and molecules [as opposed to the general notion of a small particle applied before]; for instance students account for conservation of matter during chemical transformations by paying attention to the numbers and types of atoms.

\section{Sample Learning Experiences \& Learning Performances (LE/LP) (chemical transformations)}

Explaining, predicting, developing explanatory models (cluster 1)

LE/.LP 1. Students construct models that show that a "chemical reaction" is a process in which two or more substances interact to form new substances with different properties from the old substances.

Criteria:

o Students' models should show that the new substances are made of the same atoms as the old substances, but the atoms are arranged in new ways. In addition there should be attention to the ideas that no atoms are lost or created during this rearrangement.

o Students' models should show that the number of atoms of each type is the same before and after the reaction.

LE/LP 2. Students use models to explain how a chemical reaction is similar to or different from a physical change (such as a phase change or mixing). Criteria:

o Students' models should show that in a chemical reaction the initial substances are composed of atoms arranged in unique ways before the process and then combined in new ways to form new substances after the process, while in a physical change (such as phase change or mixing) the molecular models represent the same substances before and after the process (i.e., the atoms do not rearrange).

- Students' models however should note that the numbers and type of atoms do not change before and after the transformation in either case.

Designing investigations, collecting, representing, and analyzing data (cluster 2)

LE/LP 3. Students design an investigation to determine whether a process is a chemical reaction or a physical transformation. Criteria:

o Students' designs should consider the need to identify substances based on their unique properties (should be able to draw upon a wide variety of intrinsic properties) and the need to determine if a different substance can be detected after the transformation as compared to before the transformation. Findings would show that if a chemical reaction occurred, new substances will have formed that have new properties.

o Students' designs should identify variables, and communicate scientific procedures such as the measurements of properties before and after the process.

o Students' plans should include multiple trials and measuring properties under the same conditions. 


\section{Structure, Properties, and Transformation of Matter: Level 4 \\ White background-structure \& properties of matter; Grey background - transformation of matter}

\section{Scientific argumentation (cluster 3 )}

LE/LP 4. Students construct and defend arguments from data collected and analyzed to determine whether a transformation observed is a physical transformation (such as boiling or mixing) or a chemical reaction.

Criteria:

0 Students' arguments should include a claim concerning the nature of the transformation (physical or chemical), evidence in the form of properties of the substances and/or signs of a reaction, and reasoning that (e.g.) a chemical reaction is a process in which substances interact to form new substances so that there are different substances with different properties before and after the reaction.

o Students reasoning should also rule out alternative transformations (i.e. physical transformations).

LP5. Students construct and defend an argument from data collected (or presented to them) that matter is conserved during a chemical reaction in an open system (may include data of the same reaction occurring in an open and closed system).

Criteria:

o Students' arguments should include the claim that mass is conserved during the chemical reaction and that their data supports this claim.

o Students should be able to rule out other ideas such as mass was lost in the open system after the reaction.

o Students should also apply the principle that atoms only rearrange during a chemical reaction in the reasoning of their argument. 


\section{Structure, Properties, and Transformation of Matter: Level 5 \\ White background-structure \& properties of matter; Grey background - transformation of matter}

\section{LEVEL 5 (Grades 10-12): Subatomic Model of Matter}

\section{Overview: Students explanations and learning performances have the following characteristics:}

Students hold a subatomic model of matter in which atoms are conceived of as having a substructure composed of charged particles that influence interactions within and between molecules and consequently the structure, properties and transformation of matter. Students at this level have explanations with clearer mechanisms for why atoms and molecules behave the way they do and have more refined explanations for chemical and physical transformations of matter.

\section{LEVEL 5 Student Explanation or Explanatory Model}

What is matter made of and how does its composition relate to its properties?

[Note: the following explanations are very long and were written by a chemistry educator, they would be explanations expected from a student who has completed a chemistry course in high school and has mastered the subatomic model of matter. While there is much detail and many examples to clarify thinking, examples would be needed to effectively communicate ideas at this level. Common to all the explanations is a focus on the behavior of subatomic particles, specifically electrostatic forces and principles of energy that govern the behavior of subatomic particles]

\section{Focus Question 1: What is a material or substance?}

The word "material" is quite general, and refers to any identifiable part of the concrete and tangible physical world, because all things in the physical world are made up of matter - which is a word that stands for whatever constitutes the underlying building blocks of things in the physical world. Thus, material is that which occupies space and can be perceived by the senses (or by instruments, such as microscopes and telescopes, which enhance our senses).

A substance is a specific material, one that has an identifiable and reproducible set of characteristics, or properties, that define it and distinguish it from other substances. Water is a substance because, no matter where you are in the universe, it is expected to have the same set of characteristics if it is observed under a defined set of conditions.

The matter that makes up a substance is particulate; the particles are called atoms. Chemical identity (what is the difference between atoms?) and chemical affinity (how do atoms connect, or bond, to one another?) are both explained with a simple set of sub-atomic particles (negatively charged electrons, positively charged protons, and neutral neutrons). Electrically neutral atoms are envisioned as a relatively heavy, densely packed nucleus made up of a collection of positively charged protons, in all cases except one; some neutrons, accounting for most of the mass of an atom; and a charged counter-balancing collection of low mass, diffuse electrons, with their negative charges, that surround the nucleus and account for most of the space occupied by an atom. Thus, the simplest and most abundant atom in the universe, hydrogen, is imagined to be a single proton nucleus surrounded by a single, spherically distributed electron.

There are two forces that are responsible for holding atoms together. Based on electrostatic forces, electrons repel electrons, protons repel protons, and electrons attract protons. In order to have a collection of positively charged particles (protons) all gathered together in a relatively densely packed space, along with neutrons, there must be a force that can overcome the electrostatic repulsion, and it must be strong; hence, it is called "the strong force." It is 


\section{Structure, Properties, and Transformation of Matter: Level 5}

White background-structure \& properties of matter; Grey background - transformation of matter

responsible for holding protons and neutrons together, but only operates over incredibly short distances, so electrons are not affected. Instead, the

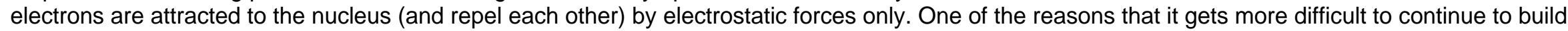

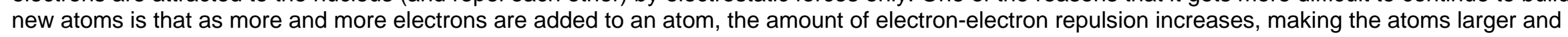
larger, and in turn causing the electrons to be farther and farther away from the attractive influence of the positively charged nucleus. Most of the large, artificial atoms have quite short lifetimes because there are not enough of the attractive forces to hold them together, and they quickly fall apart, sometimes after only a miniscule fraction of second.

\section{Focus Question 2: What happens when atoms form molecules?}

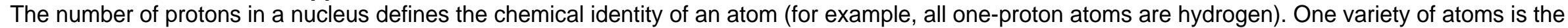
isotope, which are atoms of the same element with a different number of neutrons. As one moves from hydrogen to helium to lithium to beryllium

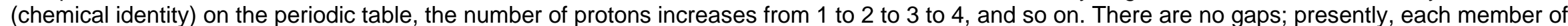

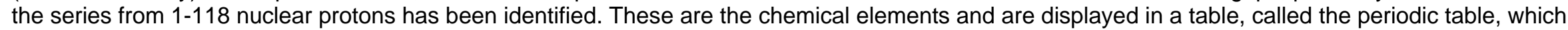
is organized by features of the elements' atomic structures.

Electrons occupy overlapping spherical shells of increasing radius. Each shell can hold a discrete number of electrons. The shell that fills first, which is

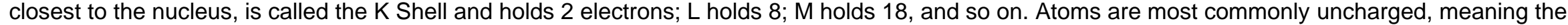

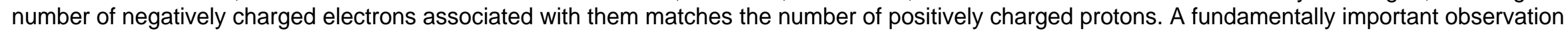
about atoms is that when a Shell is filled, that is, when the number of electrons equals the number that it can hold, the atom is relatively inert (does not easily form molecules) and generally exists as a mono-atomic entity. The exemplars are the inert gases-Helium, Neon, Argon, Krypton, Xenon, and Radon-which sit in the far right column of the periodic table. Atoms like these are said to have closed-shell electron configurations.

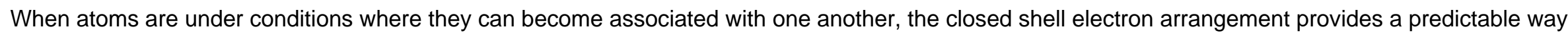

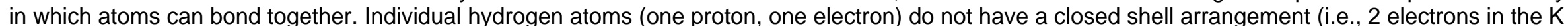

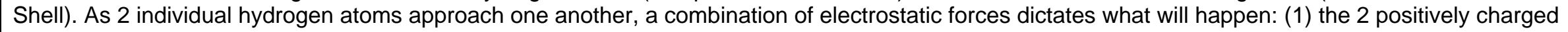
nuclei repel each other; (2) the 2 negatively charged electrons repel each other; and (3) the protons and electrons are attracted. At some distance

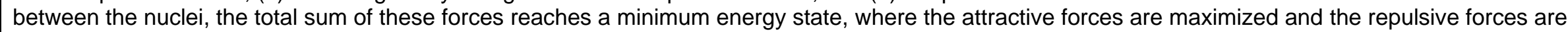

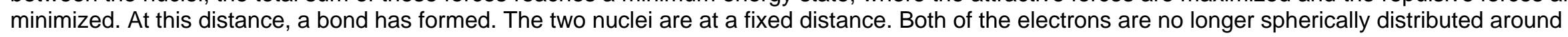

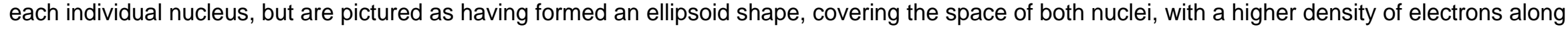
the internuclear axis (the imaginary axis that passes through the two nuclei in a bond), providing the maximum site for their attraction by the positive

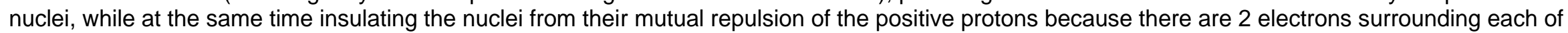
them, thus giving the appearance of a closed shell configuration to each nucleus in the diatomic (diatomic -being composed of 2 atoms) hydrogen

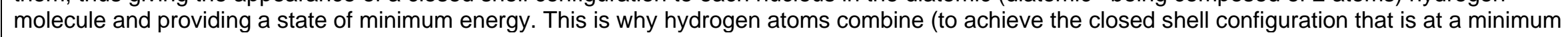
energy state) and why hydrogen molecules only involve 2 atoms of hydrogen.

Because each hydrogen atom achieves a closed shell configuration in the diatomic hydrogen molecule, the potential energy of the joined atoms (a molecule) is lower in the molecule than it was for each separate atoms. Energy, most probably in the form of thermal energy, would be released as the two hydrogen atoms bonded, sharing their electrons, and creating a more stable situation for the bonded hydrogen atoms compared to when they were individual atoms. In order to separate the atoms in the hydrogen molecule, thermal energy (for example) would have to be added.

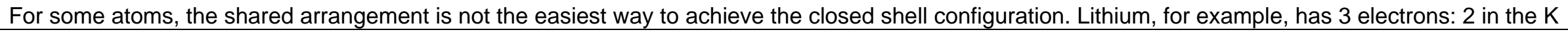




\section{Structure, Properties, and Transformation of Matter: Level 5}

White background-structure \& properties of matter; Grey background - transformation of matter

Shell and 1 in the L Shell. Electrons in an unfilled Shell are called valence electrons. In order to get to a closed shell configuration, lithium can lose an electron, thus becoming a positively charged atom, called an cation (3 protons/4 neutrons/2 electrons); otherwise, it would need to pack 7 more electrons around itself to get to the filled $L$ Shell of 8 , and this arrangement (3 protons/4 neutrons/8 electrons) would have lots of electron repulsion and not enough attractive force coming from the 3 protons to form a stable entity. Fluorine atoms, on the other hand, have just the opposite situation (9 protons/10 neutrons $/ 2 \mathrm{~K}$ electrons; $7 \mathrm{~L}$ electrons). In order to reach minimum energy of the closed $\mathrm{L}$ Shell, fluorine needs 1 electron - just like the hydrogen atoms did in forming the hydrogen molecule. Thus, two fluorine atoms can form the diatomic fluorine molecule when they get within a close enough distance in order for a pair of electrons to be shared by both of the nuclei, thereby giving each nucleus the equivalent of a filled L Shell. Alternatively, if fluorine atoms combine with lithium atoms, the fluorine atoms ( $2 \mathrm{~K}$ and $7 \mathrm{~L}$ electrons, could gain an electron to obtain the closed shell number, 8 ) can accept an electron from the lithium atoms ( $2 \mathrm{~K}$ and $1 \mathrm{~L}$ electron, can get to the closest closed shell, $2 \mathrm{~K}$, by releasing an electron). Consequently, lithium atoms ( 3 protons/4 neutrons/2K, $1 \mathrm{~L}$ electrons) will become a positively charged lithium cation (3 protons/4 neutrons/2K electrons) by the loss of an electron, while fluorine atoms (9 protons/10 neutrons/2K, $7 \mathrm{~L}$ electrons) will become the negatively charged fluoride anion (9 protons/10 neutrons/2K, $8 \mathrm{~L}$ electrons) by gaining an electron (anions are negatively charged atoms). Both atoms would reach a state of minimum energy. The resulting substance, lithium fluoride, is an ionic compound, made up of an equal, well-ordered collection of positive lithium cations and negative fluoride anions. In all of these cases, the total number and kind of particles remains constant. There is no net build-up or loss of charge, and overall, in total, any sample of molecules will be uncharged.

\section{Focus Question 3: Why do some molecules have a charge or act like they have a charge (e.g. water and sugar molecules)?}

Because all particles must be conserved, the overall charge of any given chemical system is zero. Two uncharged hydrogen atoms (a pair of 1 proton/1 electron atoms) combine to give an uncharged hydrogen molecule ( 2 protons and 2 electrons). An uncharged lithium atom ( 3 protons, 4 neutrons, 3 electrons) and an uncharged fluorine atom (9 protons, 10 neutrons, 9 electrons) give a (positive) lithium cation ( 3 protons, 4 neutrons, 2 electrons) and a (negative) fluoride anion (9 protons, 10 neutrons, 10 electrons). In this case, although each of the individual atoms carries a net charge, they are equal and opposite (involving a total of 12 protons, 14 neutrons, and 12 electrons in the system, both before and after) so that the net outcome is an uncharged system. Lithium cations and fluoride anions are called the counter-ions of an ionic compound (Li+, F-; lithium fluoride), while the two atoms in the diatomic hydrogen $(\mathrm{H}-\mathrm{H})$ or fluorine $(\mathrm{F}-\mathrm{F})$ molecules, in which the electrons are being shared, are called covalent compounds. All of these atoms have closed shell electron configurations.

In the lithium fluoride example, each of the charged entities is just an atom with an imbalance of protons and electrons, resulting in a net charge at the atomic center. There are many examples of other, complex molecules that also carry one or more charges. In every case, however, the overall system will be uncharged because the total number of particles must remain conserved.

Ions (atoms with full charges) are not limited to simple monoatomic examples. Particularly in acid-base chemistry, in which hydrogen atom nuclei (i.e., protons) are transferred from one atom with an electron pair to share (giving a closed K Shell) to another atom that can share its electron pair better (also giving another closed $\mathrm{K}$ Shell), some complex atoms centers can bear charge. In ammonium fluoride, for instance, the ammonium ion is a nitrogen atom ( 7 protons/7 neutrons/2K, $5 \mathrm{~L}$ electrons) that is bonded to 3 hydrogen atoms $(3 \times 1$ proton/ 1 electron), which closes the $\mathrm{L}$ electron shell $(5+3=8)$, but is also using a pair of its electrons to provide a bond to an additional hydrogen cation (1 proton). The entire ammonium entity, then, has 11 protons, 7 neutrons, and 10 electrons, giving an excess of a full positive charge for the ammonium ion. The overall ionic compound, ammonium fluoride, is uncharged, however, because the fluoride anion, as above, has 9 protons, 10 neutrons, and 10 electrons in its closed shell configuration. Many biological molecules, such a proteins, have uncharged atoms that can carry an additional proton, giving that atom a positive charge. Other atoms can easily carry a negative charge. Although the entire molecular entity will always be uncharged, it can be made up of atoms, which themselves, carry charge due to proton/electron imbalance at that atomic center. 


\section{Structure, Properties, and Transformation of Matter: Level 5 \\ White background-structure \& properties of matter; Grey background - transformation of matter}

In the molecular hydrogen and molecular fluorine examples, the average number of electrons around each nucleus is still the same as the number of protons, and so these atoms are uncharged. Because two atoms of the same kind make up the molecule, the number of protons in both nuclei is the same, and the electrons are symmetrically distributed because the electrostatic effects derived from both nuclei are identical. We say that the electrons are being shared equally, and that the bond is a non-polar covalent bond.

The atoms of hydrogen ( 1 proton $/ 1 \mathrm{~K}$ electron) and fluorine (9 protons $/ 10$ neutrons $/ 2 \mathrm{~K}, 7 \mathrm{~L}$ electrons) both need one more electron to achieve a closed shell electron configuration. The diatomic molecules of hydrogen $(\mathrm{H}-\mathrm{H})$ and fluorine $(\mathrm{F}-\mathrm{F})$ can accomplish this, making the shared, 2-electron bond a more stable arrangement than in the case of the isolated atoms. Atoms of hydrogen and fluorine can also share electrons with one another in order to accomplish the same goal, giving the diatomic hydrofluoric acid molecule $(\mathrm{H}-\mathrm{F})$. Because the number of protons in these two nuclei is unequal $(\mathrm{H}$ has $1, \mathrm{~F}$ has 9), the pair of electrons in the bond will be shared unequally. The electrostatic attraction of fluorine's 9-proton nucleus toward these electrons will be greater than that of hydrogen's 1-proton nucleus. Consequently, the charge distribution within the H-F molecule will be unequal. If the electrons were shared equally, as in $\mathrm{H}-\mathrm{H}$, then there is on average 1 electron always associated with each $\mathrm{H}$ atom, and this balances the positive charge of the nucleus,.. In going to $\mathrm{H}-\mathrm{F}$, there is less than 1 electron, on average, associated with the $\mathrm{H}$ end of the molecule, because of the strong attraction that the electrons have to the more positive fluorine nucleus. Thus, the positive charge of the $\mathrm{H}$ nucleus, at the $\mathrm{H}$-atom end of the $\mathrm{H}$ - $\mathrm{F}$ molecule, does not have a full negative charge (i.e., a full electron) to counter-balance it. This results in a slight excess of positive charge at the $\mathrm{H}$-atom end of the $\mathrm{H}-\mathrm{F}$ molecule. Similarly because more of the shared electron pair is located nearer to the more positive F-atom nucleus, there is an equal and opposite excess of negative charge at the F-atom end. While the overall molecule is uncharged (the total number of protons and electrons is still equal), the $\mathrm{H}-\mathrm{F}$ bond has a slight excess of positive charge, from the $\mathrm{H}$-atom nucleus, at the $\mathrm{H}$-atom end, and a slight excess of negative charge, from the electrons, at the $\mathrm{F}$-atom end. The H-F molecule is said to have a dipolar (or just "polar") covalent bond, and possess a permanent electromagnetic field, analogous with a small magnet, as a part of its chemical structure.

Whenever non-identical atoms are bonded, the bond is dipolar, with more of the electrons associated with the atom whose nucleus exerts a stronger electrostatic attraction. An extremely important exemplar is water as the polarity of water accounts for many of it properties. In water molecules, where two hydrogen atoms are each bonded to a central oxygen atom $(\mathrm{H}-\mathrm{O}-\mathrm{H})$, both of the $\mathrm{H}$-O bonds are polar covalent. The molecule is uncharged because the total number of electrons matched the number of protons. Once a molecule has more than 2 atoms, however, molecular geometry dictates whether or not the molecule itself will have a dipolar character. If $\mathrm{H}-\mathrm{O}-\mathrm{H}$ is linear (180 degrees), then the two bond dipoles are equal and exactly opposite from one another, and in this geometry, they would cancel out and leave no overall molecular Dipole Moment (a completely symmetrical electromagnetic field, with two little excess positives are the ends, and some excess negative in the middle). This linear water molecule would be non-polar. However, if the $\mathrm{H}$-O- $\mathrm{H}$ geometry is anything other than 180 degrees (the overall shape of the molecule would be like the letter "V" with H's at the end and the O at the vertex), then the two $\mathrm{O}-\mathrm{H}$ bond dipoles are not sitting exactly opposite from one another, and so the overall molecular Dipole Moment will be the water molecule with a slightly excess positive charge being contributed by the $\mathrm{H}$ 's, and a slightly excess negative contributed by the $\mathrm{O}$. The resulting electromagnetic field for the molecule is not as symmetrical as for the 180-degree case, and so the molecule itself has two clearly different regions: the positive end, where the hydrogen's are, and the negative end, where the oxygen is. Consequently, the overall molecule would possess a permanent electromagnetic field, with a slight negative charge associated with the oxygen atom and slight positive charges associated with the hydrogen atoms. The vast majority of molecules are asymmetrical, and are made up of dipolar covalent bonds, which means that the molecules, although uncharged overall, are scattered with regions of slightly positively and negatively charged regions that can vary in intensity.

Focus Question 4: Why are some elements so reactive or nonreactive (e.g. hydrogen versus helium)? For the vast majority of molecules, reactivity is made up of two components. First, there are other favorable combinations of an atom or molecule with 


\section{Structure, Properties, and Transformation of Matter: Level 5}

White background-structure \& properties of matter; Grey background - transformation of matter

other atoms and molecules, compared to if they where simply separated from one another. This could be a combination that results in a better arrangement, that is, an arrangement where the electrons are shared more equally with the available nuclei, thus making a more favorable set of electrostatic interactions, but this is not a necessary requirement. There simply needs to be another arrangement of the atoms in which there are some favorable interactions relative to being separated as individual atoms. Second, the conditions under which atoms and molecules exist need to allow this reorganization to take place. For example, it is quite favorable for diatomic hydrogen molecules to react with diatomic oxygen molecules to form water molecules. When this happens, the release of thermal energy associated with getting to the more stable electrostatic arrangement is quite high (an

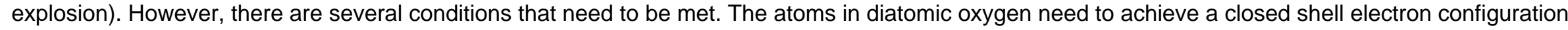

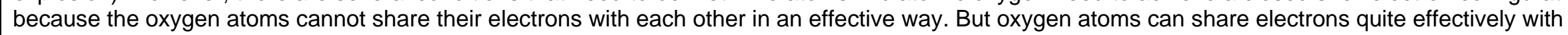
hydrogen atoms, and so the energy minimum for the atoms in water molecules is significantly lower than when the atoms are in the diatomic hydrogen and oxygen molecules. But this is not enough. Some energy needs to be added to the system in order to initially break the bonds in the oxygen and

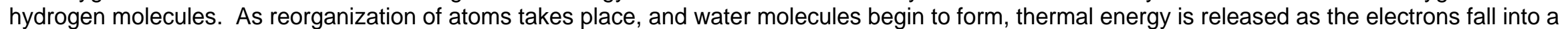
more favorable electrostatic arrangement in the $\mathrm{O}-\mathrm{H}$ bonds. This thermal energy is enough to continue to break apart more hydrogen molecules into hydrogen atoms, and so the reaction process can continue. Although atoms in water molecules can form hydrogen and oxygen gases, a great deal of energy is needed in order to accomplish that and it is likely that they would recombine to form water molecules.

If the first condition is not met, that is, if there is not a more energetically favorable arrangement for the electrons to achieve, then no reactions will take place, because the starting condition is the best arrangement. Atoms of helium, neon, argon, and the other atoms in the far right column of the periodic table, all have an ideal arrangement. Located on one atom, in a perfectly spherical distribution around a positive nucleus, there exists the number of

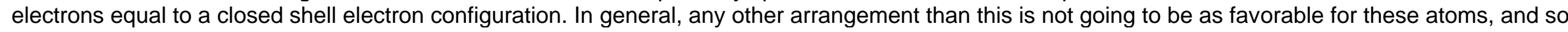
they are generally unreactive. But if the first condition is met, molecules need to collide with sufficient energy to initially break the bonds to allow a reassembling of the atoms into more energetically favorable arrangements.

\section{What has changed in students' thinking from the previous level?}

o Explanations and models for how atoms and molecules behave include clearer and more refined mechanisms including the addition of subatomic particles and how they interact.

o Students' view of matter now includes the idea that atoms have a substructure with charged sub-particles and that these particles influence how atoms interact with each other to determine how atoms form molecules and also how molecules interact with other molecules. An understanding of the attractive and repulsive forces due to charges associated with electrons and protons enable students to provide a clearer mechanism in explanations and models for why atoms and molecules behave the way they do. Electrons should have particular prominence in explanations for why and how atoms interact with each other.

o In these explanations there is an understanding that the system of atoms involved in the collection of molecules move towards a state of lower energy.

o Now that students have more experience distinguishing atoms from molecules they should be able to include specific example of molecules or atoms that help to articulate their thinking about the behavior of atoms and its subatomic particles in their explanations. 


\section{Structure, Properties, and Transformation of Matter: Level 5 \\ White background-structure \& properties of matter; Grey background - transformation of matter}

\section{Student Ideas \& Boundaries}

\section{Prior Ideas, Naive Conceptions, Challenges}

Atomic Structure

Students may hold a number of conceptions that need to be considered when learning about these concepts. Students may believe that:

- A charged object can only contain one kind of charge (Horton, 2007).

- Classical mechanics effectively and accurately predicts and describes the behavior of matter under all conditions and scales (Kalkanis, Hadzidaki, \& Stavrou, 2003).

- Electrons move around the nucleus in orbitals like planets around the solar system (Griffiths \& Preston 1992; Unal \& Zollman, 1999).

- An electron cloud consists of a mass of suspended electrons much like water droplets in a cloud above the earth (Horton, 2007).

- Atoms containing equal numbers of protons and neutrons are more stable.

- Neutrons neutralize the repulsion between protons within the atomic nucleus.

\section{$\underline{\text { Interactions }}$}

Before and as they learn about these ideas, students may believe that:

- Electrons between two bonded atoms spend most of their time equidistant between the atoms (Taber and Coll 2002).

- Bonded materials must always be in the form of molecules (Pallant and Tinker 2004).

- Interactions between charges results in neutralization, not the formation of bonds (Pallant and Tinker 2004).

- Ionic solids consist of molecules arranged in a pattern and stabilized by intermolecular interactions (Pallant and Tinker 2004).

Secondary students, including high school students, are challenged to connect representations of the microscopic world of matter as they relate to macroscopic phenomena involving matter (Treagust and Chittleborough et al., 2003).

\section{Useful or Productive Ideas}

A focus on the model of the interactions between atoms instead of a calculation and classification of the "type" of interaction may help prevent memorization of categories and classifications and help students consider the interactions as part of a continuum.

\section{Boundaries}

The quantum mechanical model of electron distributions in atoms used at this level is a generalized and simplified description, and no attempt to fully describe or explain it is made.

Bond angles: The structure of the water molecule was briefly discussed, but the detailed explanation of why the water molecule has this structure is beyond the scope of this level. However, it is important for students to know that molecules do have a structure and that this structure is due to the electrostatic interactions of the atoms in the molecule. The structure of the atom often helps account for the polarity of molecules.

\section{Rationale and Notes}




\section{Structure, Properties, and Transformation of Matter: Level 5 White background-structure \& properties of matter; Grey background - transformation of matter}

o These highly sophisticated explanations are meant to represent the explanations of students who have completed a high school course in chemistry and have mastered the subatomic model of matter. While the specific examples used to articulate ideas are not essential to the explanations, examples are seen as helpful to articulating students' ideas. More important are ideas about the structure and behavior of the subatomic particles and the electrostatic forces and principles of energy that govern their behavior.

o We chose to organize students' explanations around focus questions as done previously at earlier levels to show more clearly how students' explanations or models are changing relative to the previous level, and we considered phenomena that are both relevant to students lives and that are frequently encountered in multiple scientific disciplines (e.g. relative reactivity of different elements, charged substances, and the structure of water, as its polarity explains much of its properties); therefore these are important phenomena involving matter that students should have explanations or models for.

o Knowing ideas about subatomic structure of atoms and how atoms bond together to form more stable structures with relative minimum energy will also support future learning about the structure and behavior of matter explored in advanced coursework in chemistry or physics, including ideas in molecular biology such as the structure of DNA and proteins.

\section{Notes on how students demonstrate and apply their knowledge (as reflected in Learning Performances)}

o Phenomena that students explain, investigate, and argue about include properties like polarity and charge (which represent a new set or properties that can be accounted for).

o In explaining or constructing arguments, students can use and apply ideas of sub-atomic charged particles and the electrostatic interactions that result [as opposed to the general notion of "attractive forces" noted before].

o In critiquing others models or explanations students can describe clearer mechanisms that explain the behavior of atoms and molecules now that electrostatic forces and principles of energy [i.e. a tendency of system of atoms to move toward a state of lower energy] are included in the explanations.

\section{Sample Learning Experiences \& Learning Performances (LE/LP)}

LE/LP1. Use subatomic models to explain why one substance is different from another substance and has different properties. Criteria:

o Students should use the ideas of electrons, protons and neutrons to describe the structure of different atoms of different substances.

LE.LP2. Use a subatomic model to explain why some atoms are more unstable than others and likely to decay more easily than others. Criteria:

o Students should apply a subatomic model that includes a dense nucleus with protons, neutrons, and electrons, and appropriate 


\section{Structure, Properties, and Transformation of Matter: Level 5}

White background-structure \& properties of matter; Grey background - transformation of matter

electrostatic forces between these particles, to account for physical differences (e.g. differences in mass reflect mainly the differences in the number of protons and neutrons in the nucleus of the atoms, or some atoms fall apart more easily because larger atoms with more protons have more electrons and as more electrons are added they repel one another and are farther away from the nucleus resulting in a weaker attractive forces which cannot hold the atoms together).

LE/LP3. Build models to show why some atoms will bond together to form new substances and other atoms will not bond together. Criteria:

o For molecules that contain different types of atoms (e.g. water, $\mathrm{HF}, \mathrm{HCl}$ ) students should identify electrostatic forces at play that determine the charged properties of the material. Students' models should describe electrons being transferred and not protons.

o Students' models should include the idea that negative charges on the electrons and positive charges on the protons influence the distribution of electrons shared between bonded atoms and results in partial and opposite charges about the nuclei of the different atoms in the molecule.

o In atoms that don't bond together, student models should show that electrostatic repulsion would be greater than the attractive pull of the protons.

LE/LP4.: Construct an explanation for why some elements are reactive (e.g. hydrogen) and others are not (e.g. helium).

Criteria:

o The explanation should consider the state of the atom involved (e.g. does the atom have filled outer shells and is it in a favorable state), and whether there is an arrangement of the atoms in which there are some favorable interactions relative to being separated as individual atoms. Depending on the specific element used in the instructional task or assessment, students may also need to account for other conditions such as the availability of heat energy to break bonds between atoms.

LE.LP5. Construct models to show why some molecules are polar and others are non-polar.

Criteria:

o The explanation should include why identical atoms bonding together are non-polar and when non-identical atoms bond together they are polar. Whenever identical atoms bond with each other, the electrical charge associate with the molecule is equal, or non-polar because the nuclei of both atoms attract the electrons of both atoms equally. Whenever non-identical atoms are bonded, the bond could have an unequal attraction of electrons because the atom whose nucleus exerts a stronger electrostatic attraction will attract the electrons more so than the atom that does not exert as great of an attraction. However, a second factor also is important and that is molecular geometry. Molecules that have non-identical atoms and that have angles will be polar but if molecules are linear like that of carbon dioxide, it will be non-polar.

\section{Designing investigations, collecting, representing, and analyzing data (cluster 2)}

LE/LP 6: Students use their knowledge of what elements will likely react together to form new substance, provide an explanation based upon their atomic structures and then describe an experiment to form the new material.

Criteria:

o Explanations include the following: a) atoms or molecules react together to form new molecules in which minimum energy states exist (this involves forming closed shells), b) students should further argue that because new molecules where formed a new substance was produced that would have different properties than the reacting materials. 


\section{Structure, Properties, and Transformation of Matter: Level 5 \\ White background-structure \& properties of matter; Grey background - transformation of matter}

$0 \quad$ Students should design experiments to collect properties of the reacting and starting materials to show that they have different properties. Properties would include both physical and chemical.

Constructing scientific arguments (cluster 3 )

LE/LP6 : Critique different explanations of how electrical interactions affect the properties and function of a given molecular structure (structures could include water molecules, noble gasses, proteins, DNA).

Criteria:

Student critiques should include a description of the type(s) of interactions that are occurring within the structure with a justification that relates to the structure in question and how these interactions affect the properties of the substance. For instance, because of the polarity of water, it will have a high boiling point. Where appropriate, the justification may include properties and characteristics predicted by the periodic table.

\section{LEVEL 5 Student Explanation or Exploratory Model (physical transformations)}

What is taking place when matter changes?

Focus Question 1: What happens when something dissolves in water?

Water molecules, in the liquid state, are constantly being jostled because of their electrostatic properties and their structure. There are two distinct groups of water molecules in the liquid state: those at the surface, and those in the interior, or bulk, of the liquid. The molecules in the interior can interact, via electrostatic interactions, with their neighboring molecules, in an infinite number of arrangements. The slightly positive end of one water molecule is attracted to the slightly negative end of another, and so on. And no matter how that molecule spins or moves, it finds itself in the presence of other partners with which it can be organized in a favorable electrostatic arrangement. The molecules at the surface of water, on the other hand, are not in a favorable electrostatic arrangement. In one hemisphere of space surrounding a surface water molecule (the water hemisphere) there are plenty of other water molecules to interact with, just as in the interior. But in the other hemisphere, perhaps this is a water droplet in the air, there are no other water molecules, just the vast openness of the open space where the sparse distribution of gas-phase molecules that make up the air are located. As a result, the water molecules on the surface of water are more highly organized and ordered than the ones in the interior in order to be able to receive the benefit of the electrostatic interaction with other water molecules. Water, therefore, behaves in a way to minimize its surface area, because the greater the surface of water, the more molecules there are that are not getting the energy benefit from electrostatic stabilization.

Dissolving means that two things are mixed and they stay mixed. Just as in a chemical reaction, the mixed state must be more stable than the unmixed state in order for a substance to dissolve in water. The interior of water, as described above, is quite favorable for water molecules because of the high number of electrostatic attractions that are possible. Thus, in order for a substance to dissolve in water, that substance needs to be able to replace, or at least compensate for, the electrostatic stabilization that water molecules have with one another. As a result, molecules that have the same kinds of charges and partial charges as water molecules can replace them in the bulk interior of water, and we say that they have dissolved, that is, they are intimately mixed with water molecules.

Most ionic compounds, because they are made up of fully charged ions that can interact well, electrostatically, with the polar ends of water molecules, can mix (dissolve) well. Molecules that are like water, and have a high fraction of polar covalent bonds in their structures, can also dissolve readily in water because the partial charges in those polar covalent bonds can provide favorable electrostatic interactions for the water molecules. Additionally, molecules in which $\mathrm{O}-\mathrm{H}$ and $\mathrm{N}-\mathrm{H}$ bonds dominate their chemical structure, readily dissolve in water, because these types of bonds are particularly polar (high partial 


\section{Structure, Properties, and Transformation of Matter: Level 5 White background-structure \& properties of matter; Grey background - transformation of matter}

charges).

If a molecule is not ionic, or it does not have polar covalent bonds, it generally does not dissolve in water. Gasoline, for instance, is made up of molecules with no polar covalent bonds, and it simply floats on top of water. Even when a water and gasoline mixture is agitated in order to try to get mixing to take place, the mixture will rapidly un-mix to give gasoline floating on the water again. When the molecules in gasoline are forcibly inserted into the interior of water, those nearby water molecules lose some of the electrostatic stabilization they had when other water molecules were nearby. The water molecules near the molecules from gasoline are more organized and now have fewer electrostatic interactions. This is not favorable for the water molecules, which will be more stable when there are more sources of electrostatic interaction nearby. Consequently, the water and gasoline un-mixes, and this creates the least amount of surface area for the water molecules as possible.

\section{Focus Question 2: What happens when something boils?}

The molecules of a substance in the liquid state can move about, but are attracted to neighboring molecules through an electromagnetic interaction. The interaction arises because electrons in the atoms of one molecule are attracted to the positive charges in the atoms of neighboring molecules. As the temperature of the substance rises, the average kinetic energy of the molecules rises. Since the molecules have a range of kinetic energies, at any temperature those molecules at the surface with the greatest kinetic energies can overcome the attractive electromagnetic forces of the nearby molecules and escape into the space above the liquid surface. When this happens the molecules constitute the substance in the gas (vapor) state, and the process is called evaporation.

When the temperature becomes sufficiently high, molecules whose kinetic energy are at or above the average value have sufficient kinetic energy to escape the attractive electromagnetic forces of neighboring molecules throughout the entire liquid and escape into a space called a vapor bubble. Because the vapor bubbles have a much smaller density than the surrounding liquid they immediately rise to the surface. Molecules inside the bubble constitute the gas or vapor state of the substance. When this happens the process is called boiling, and the temperature at which it occurs is called the boiling point.

Another factor influencing boiling point is the mass of the atoms or molecules. For example, for non-polar molecules, the mass is the determining factor to explain boiling point. Heavier molecules have higher boiling points.

\section{What has changed in students' thinking from the previous level?}

o Students' explanations for physical changes include clearer and more refined mechanisms for why molecules interact and behave the way they do because now the "attractive forces" - as referred to at the earlier levels -- are now conceived of as electrostatic forces resulting from interactions between charged subatomic particles.

\section{Student Ideas and Boundaries (physical transformations)}

Prior Ideas, Naive Conceptions, Challenges

Some high school students may think that bubbles from boiling water consist of air, or oxygen gas, or hydrogen gas (Osborn and Roger et al., 1983; Schmidt, 1997; Bodner, 1991). 


\section{Structure, Properties, and Transformation of Matter: Level 5 White background-structure \& properties of matter; Grey background - transformation of matter}

Some students may think that boiling, or other phase changes, are examples of chemical reactions [Ahtee and Varjola, 1998). Intra-molecular bonds are broken when substances change phase - including boiling (Gensler, 1970).

Some student may think that during dissolving sugar melts, and becomes liquid sugar (Abraham and Bryzbowski, 1992); or that salt, and sugar disappears when dissolving (Mulford, 1996; Lee and Einchenger et al, 1993).

\section{Boundaries}

At this point entropy is not considered.

\section{Rationale and Notes}

o We chose to organize students' explanations around focus questions as done previously at earlier levels to show more clearly how students' explanations or models are changing relative to the previous level, and we considered phenomena that are both relevant to students lives and are frequently encountered in phenomena studied in other disciplines (e.g. solutes dissolving in water and phase changes from liquid to gas); therefore, they are important phenomena involving matter that students should have explanations for.

o We recognize these are not complete explanations because concepts such are entropy are not considered and may be beyond the scope of this progression -- and the NRC framework (they may be more appropriate for AP chemistry). However, even though these explanations may be somewhat incomplete, they represent progress in thinking and more sophisticated explanations that could be leverage for the more complete explanations developed later (perhaps in AP chemistry or college).

\section{Notes on How students demonstrate and apply their knowledge (reflected in Learning Performances)}

o In explaining phenomena or critiquing others' models students can use and apply ideas of sub-atomic charged particles and the electrostatic forces that result from those interactions [as opposed to general notions of "attractive forces" mentioned at the previous level]. Students can also apply principles of energy to the system of atoms or molecules [i.e. a tendency of system of particles to move toward a state of lower energy].

\section{Sample Learning Experiences \& Learning Performances (LE/LP) (physical transformations)}

Explaining, predicting, developing explanatory models (cluster 1)

LE/LP1. Draw a model to explain what happens when something dissolves in water and why other substances won't dissolve in water. Criteria:

o Students' models should include electrostatic interactions between neighboring molecules and water molecules in which slightly positive ends of water molecules are attracted to slightly negative ends of molecules of a substance dissolved in water and vice versa for the slightly oppositely charged ends of water and solute molecules. 


\section{Structure, Properties, and Transformation of Matter: Level 5}

White background-structure \& properties of matter; Grey background - transformation of matter

LE/LP2. Construct an explanation for what happens when something boils.

Criteria:

o Students' explanations should include ideas of kinetic energy of molecules increasing with increasing temperature and of the kinetic energy overcoming attractive electromagnetic forces.

o Students' explanations should attend to variation in kinetic energy of individual molecules within the system of molecules in the liquid sample - especially those at the surface of the liquid sample of those within vapor bubbles.

Designing investigations, collecting, representing, and analyzing data (cluster 2)

LE/LP3. Collect measurements of the solubility of different substances in water and use the polarity of these molecules to account for the relative solubility.

Criteria:

o Students should realize those substances that are capable of dissolving in water are polar (and do not share electrons equally between atoms bound together in the molecules that constitute the solute).

Constructing scientific Arguments (cluster 3)

LE/LP 4. Critique a peer's explanation for why one thing dissolves in water (e.g. sugar or NaCl) but not another thing (e.g. gasoline).

Criteria:

0 Students' critiques should consider whether the explanation includes electrostatic interactions between water and the solute molecules and whether the solute molecules are polar like water (i.e. have partially charged sides to the molecule) or involve ionic bonds.

o Students should also point out any inconsistencies in other students' explanations that suggest a chemical reaction is taking place.

LE/LP 5. Critique explanations for why one substance has a higher boiling point than another substance. Criteria:

o Student critiques should consider two primary factors that determine boiling point: 1) electrostatic interactions among particles and 2) mass of a molecule. For non-polar molecules, the mass is the determining factor to explain boiling point. Heavier molecules have higher boiling points. For example, oxygen has a higher boiling point than hydrogen because oxygen is a heavier metal. For other substances electrostatic interactions become the dominant factor. For a polar molecule, its polarity becomes the dominant factor in explaining the boiling point. For instance, water has a much high boiling point than sulfur dioxide because water has greater polarity. Molecules with greater polarity have stronger electrostatic attractions, thus it takes more energy to break the attraction among molecule from the other.*

*Note this explanation is not necessarily the most comprehensive critique for differences in boiling point among different substances, because there are other electrostatic interactions to consider because of special properties of atoms or molecules that make up substances (e.g., metals have delocalized shared electrons that would need to be considered to be a more complete critique). We would expect students to have more comprehensive critiques in advanced course work such as AP chemistry when students can consider more special and unique properties of substances. Nevertheless, a consideration of the electrostatic interactions would be expected in general at level 5 in learning performances such as these.

Level 5 Student Explanation or Explanatory Model (chemical transformations): 


\section{Structure, Properties, and Transformation of Matter: Level 5}

White background-structure \& properties of matter; Grey background - transformation of matter

What is taking place when matter changes?

\section{Focus Question 1: What happens when a chemical reaction occurs?}

In order for a chemical reaction to occur, energy must be added to break the interactions that hold the atoms of the reactants together, thus increasing the potential energy of the separated atoms. In the reaction mixture, the starting molecules, or reactants, must collide with each other with the right orientation and enough kinetic energy in order for the reaction to occur. When products are formed, atoms interact to reach a state with lower potential energy and energy is released in the process.

In a chemical reaction, molecular forms you begin with not only separate from one another, they also recombine into new forms as well as form back into the original structural arrangement. In chemical reactions, once energy is provided to move the atoms in the starting molecules away from their favorable bonding arrangements, there are generally many different options for how these atoms can recombine. It is rare when complex molecules undergo chemical reactions to give only one outcome; there are often many options for how the atoms might combine.

In complex molecules, not all of the atoms separate from one another. This would create a nearly infinite collection of molecular outcomes. Instead, it is generally the weakest bond, that is, the one with the least electrostatic stability in the molecule, which breaks. When the recombination of atoms and molecular pieces takes place, some of the resulting arrangements might be more stable and some might be less stable. Even a less stable arrangement can be the observed product from a chemical reaction because these new molecules form under conditions where, for instance, there is simply not enough energy around to break them back up. As a result, the outcome from a chemical reaction is not required to be the most stable arrangement of the atoms that is possible, only that arrangement which was least able to return to the reactive state of disconnected atoms. For instance, a less stable arrangement of atoms might precipitate from the solution, which might itself be a highly favorable process, and so these new molecules are simply not available for continued reaction. Another thing that might happen is that a new, less stable arrangement of atoms escapes the reaction flask because it goes into the gas phase, and also becomes unavailable for further reactions. In these cases, the overall energy of the system is still lower, afterwards, than it was beforehand, but there was a complex set of occurrences, not just the chemical reaction itself, which took place.

Sometimes, a chemical reaction forms new products that are more or less stable than the starting compounds, and the conditions are right for those products to break back up and reform the starting compounds. And, in turn, the conditions are still right for the original reactions to happen again. And so on, back and forth, back and forth. As long as there is available thermal energy, this back and forth process can continue until the experiment stops it. At that point, the distribution of starting materials and products would simply represent the natural distribution based on the relative stability of the different components.

\section{Focus Question 2: What influences reaction rates?}

A number of factors influence the rate at which chemicals react. A few important factors are the temperature, pressure, and concentration of reactants and products and reactant. Temperature, pressure and concentration relate to the motion of the particles in a system, which in turn affects the rate of collisions that occur between particles and therefore the way the particles interact, because it is more likely that the reacting molecules will have enough energy and appropriate orientation to react. However, while the conditions determine the average motion of the particles in a sample, not every individual particle has the same amount of kinetic energy and therefore is not moving at the exact same speed as every other particle. This explains why some collisions between particles have enough energy to initiate a chemical reaction while others don't.

Temperature, pressure, concentration affect the rate and direction (i.e. relative rates of the forward and reverse reactions) of a chemical reaction or whether it will even occur. How these factors affect the direction and rate of the reaction depend on the energy needed to break atoms apart from the 


\section{Structure, Properties, and Transformation of Matter: Level $\mathbf{5}$ \\ White background-structure \& properties of matter; Grey background - transformation of matter}

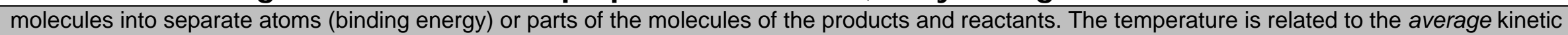

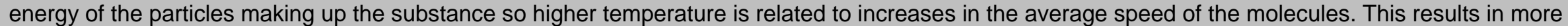

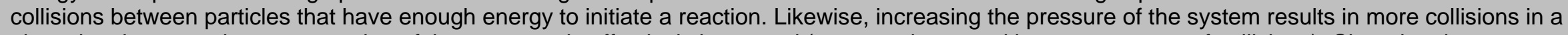
given time because the concentration of the reactants is effectively increased (pressure is caused by a greater rate of collisions). Changing the concentration of one or more of the reactants can also increase the chances of the molecules colliding in a productive manner.

\section{Focus Question 3: Why do some reaction release energy and others do not?}

When a chemical reaction takes place, enough energy has been added in order to reorganize some of the atoms. In general, energy needs to be added to get some bonds broken, and then energy is released as new bonds form. The question is one of balance and energy inventory. Similarly, the formation of new bonds will invariably release energy, but that energy might well not be equivalent to the energy that was needed to break the bonds, or it might be much more.

Reactions often involve a complex sequence of changes in which bonds are broken and formed, where molecular shape changes, where the interactions with solvent molecules is affected, and subsequent reorganizations take place which can only occur after the first ones have been completed. In comparing the relative stability of only the starting materials and the products, there are times when the desired product molecules, that is, the organization of just those atoms, is less stable than those of the particular starting material. But it is rarely true that only the atoms of the starting material end up as the atoms of the product. Instead, multiple different molecules have had constituent parts broken and recombined into multiple new forms.

In some cases, the overall transformation results in a collection of new bonds, in the products, that are all clearly much more stable than the starting materials. This overall stabilization of the electrons by their more favorable new interactions with these nuclei is accompanied by the obvious release of energy, such as the reaction heating up or giving off light. These reactions still took in energy from the surroundings in order to get going, and some part of the energy that was released might well be absorbed by the reaction as it continues. Combustion reactions are a good example.

All processes absorb thermal energy from their surroundings in order to allow a transformation to take place. Sometimes there is obvious thermal or light energy released as the reaction occurs because the products are significantly more stable. Combustion, and in the extreme, explosions, are a testimony to this. Other times, however, the main reaction products are less stable than the starting materials. Not only is less thermal energy given off than is absorbed, but in the case where product molecules go into the gas phase, even more energy can be absorbed from the surroundings. In some of these cases, the reaction vessel might actually cool down as thermal energy is pulled from the reaction system faster than it can absorbed from the overall surroundings.

\section{What has changed in students' thinking from the previous level?}

o Students' explanations for chemical changes include clearer and more refined mechanisms for why atoms interact and behave the way they do during chemical reactions because electrostatic forces are included, as well as ideas about the tendency of the system of molecules to reach a lower energy state.

- Having developed an initial understanding of chemical reactions at the previous level, level 4, students are able to consider the details of reactions, such as rate of reaction or difference in net energy released or absorbed during a chemical reaction.

\section{Student Ideas \& Boundaries (chemical transformations)}




\section{Structure, Properties, and Transformation of Matter: Level 5 \\ White background-structure \& properties of matter; Grey background - transformation of matter}

\section{Prior Ideas, Naive Conceptions, Challenges}

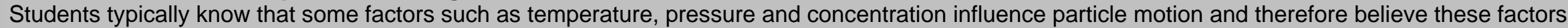
also influence rate of reaction. They may, however, reverse relationships. (e.g., Cakmakci, et al., 2003; Çalık \& Ayas, 2005).

\section{Chemical Reactions:}

- Few high school students consider ideas such as energy and stability when explaining chemical reactions (Cokelez, et al., 2008).

- Energy is used up in chemical reactions (Kesidou and Duit, et al., 1993).

Students may believe that:

- Atoms need "filled shells" or that chemical reactions occur so that atoms can obtain filled shells (Taber, \& Coll, 2002).

- Energy is released when a bond breaks and is required when bonds form (Boo \& Watson, 2000).

- All reactions always go to completion.

\section{Reaction rates:}

Some students may believe that:

- Reactions that take place at the same temperature have equal kinetic energy, thus the rate of reactions would be the same (e.g. Cakmakci, et al., 2003).

- Students may confuse extent of reaction and the rate of reaction (van Driel \& Graber, 2001).

\section{Useful or Productive Ideas (levers or stepping stones)}

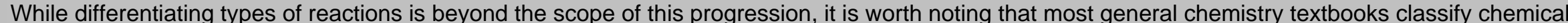
reactions as synthesis, decomposition, single displacement, double displacement, and combustion, this classification is not general. For example, this

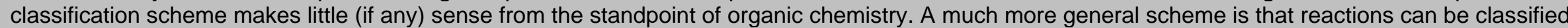

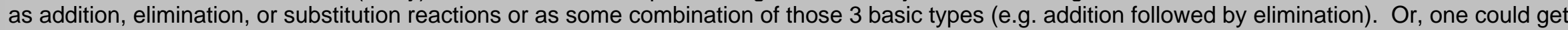
even more general and classify every reaction as an oxidation or reduction (which might have its own advantages as far as getting students to see

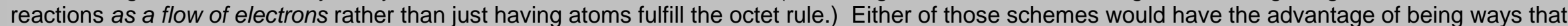

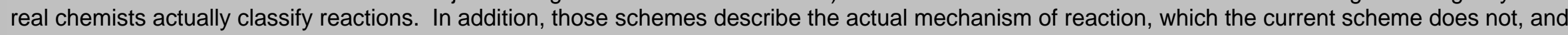

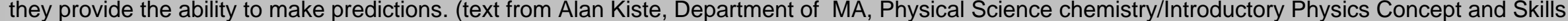
Progressions, 2010)

A useful organizing principle for chemical bonding and reactions is the notion of "structure and reactivity." (Ege, Coppola, \& Lawton, 1997). That is, the structures of all compounds determine their properties and reactivity. And, one can predict the properties and reactivity of new materials by examining

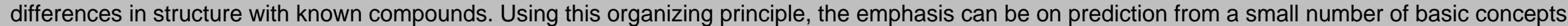
(e.g. electronegativity and VSEPR) rather than memorization of the properties and reactivities of a large number of compounds. (text from Alan Kiste, Department of MA, Physical Science chemistry/Introductory Physics Concept and Skills Progressions, 2010)

\section{Boundaries}

At this point entropy is not considered. 


\section{Structure, Properties, and Transformation of Matter: Level 5}

White background-structure \& properties of matter; Grey background - transformation of matter

At this level, students do not calculate binding energies of reactants and products.

\section{Rationale and Notes}

o We chose to organize students' explanations around focus questions as done previously at earlier levels to show more clearly how students' explanations or models are changing relative to the previous level, and we considered phenomena that are both relevant to students lives and are frequently encountered in other disciplines (e.g. endothermic verses exothermic reactions and factors that influence reaction rates ); therefore, they are important phenomena involving matter that students should have explanations for.

- We recognize these are not complete explanations because concepts such as entropy are not considered and may be beyond the scope of this progression -- and the NRC framework (they may be more appropriate for AP chemistry). However, even though these explanations may be somewhat incomplete, they represent progress in thinking and more sophisticated explanations that could be leverage for the more complete explanations developed later (perhaps in AP chemistry or college).

\section{Notes on how students demonstrate and apply their knowledge (as reflected in Learning Performances)}

o Students are able to account for the details of chemical reaction, such as differences in reaction rates influenced by factors such as temperature and concentration of reactants and products; and therefore can account for a wider range of observations about chemical reactions.

o In explaining phenomena or critiquing others' models students can use and apply ideas of sub-atomic charged particle and the electrostatic interactions that result from those interactions and have clearer and more refined mechanisms for why atoms behave as they do during a chemical reaction. Students can also apply principles of energy to the system of atoms or molecules [i.e. a tendency of system of particles to move toward a state of lower energy]

\section{Sample Learning Experiences \& Learning Performances (LE/LP) (chemical transformations)}

Explaining, predicting, developing explanatory models (cluster 1)

LE/LP 1. Provide a general explanation of what happens in a chemical reaction. Criteria:

o Students should discuss that energy needs to be added to the system to break the bonds in the starting molecules and that when products form, thermal energy is released. Thermal energy is necessary to allow collision between molecules to be more effective in breaking bonds.

o Students should also bring in the idea of equilibrium here - that forward and reverse processes are occurring.

LE/LP 2. Provide an explanation of why some reactions release energy but others don't. Criteria:

o Students should state that energy needs to be added to the system to break the bonds in the starting molecules and that when products form, thermal energy is released. The initial energy is necessary to allow for collisions that will break bonds. When the atoms reassemble, 


\section{Structure, Properties, and Transformation of Matter: Level 5}

White background-structure \& properties of matter; Grey background - transformation of matter

energy is released. Typically more energy is released than absorbed because a more stable, lower energy state is achieved, i.e., new products are at a minimum energy state that is lower than the beginning reactants. However, there are cases where less energy is released than absorbed and this results in the reaction being cooler or energy being absorbed from the environment. This typically happens when there is not enough energy to cause the products to react in the opposite direction or if one of the products is removed from the system by precipitation or escaping into the gaseous phase. Additionally, if products escape into the gaseous state, this will result in further cooling or absorption of energy.

LE/LP 3. Explain why changing the conditions (temperature, pressure or concentration) can affect the forward/reverse rate of a reaction. Criteria:

o The explanation applies collision theory to explain how a change in temperature, pressure, or concentration increases or decreases the number and intensity of collisions that result in subsequent changes to the reaction rate in a particular direction. The explanation may indicate that a balance of forward and reverse reactions may be achieved once the system has adjusted to the change in conditions (equilibrium).

Designing investigations, collecting, representing, and analyzing data (cluster 2)

LE/LP 4. Analyze and interpret a data set in which different variables were changed when two different substances were mixed that can undergo a chemical reaction. The data set includes changes in temperature, and concentration of reactants and products, and pressure (can give data from a virtual experiment if don't have equipment to change pressure). Data examines rate of reactions by measuring changes in amount of reactants or products over time.

Criteria:

o Students identify patterns in which an increase in temperature, pressure or concentration of reactants increases rate of reaction or the inverse relationships.

Scientific argumentation (cluster 3)

LE/LP5. Critique an incorrect explanation for why some reactions release energy and others do not (e.g. when bonds break energy is released)

Criteria:

o Student critiques should consider ideas such as the net difference between energy required to break bonds and the energy released when bonds form , and whether the products of the reaction are more stable than the reactants as a result of more favorable electrostatic interactions. 


\section{Structure, Properties, and Transformation of Matter}

\section{References}

Abraham, M.R., Grzybowski, E.B., Renner, L.W., \& Marek, E.A. (1992). Understandings and misunderstandings of eighth graders of five chemistry concepts found in textbooks, Journal of Research in Science Teaching 29, 105-120.

Ahtee, M. \& I. Varjola (1998). "Students' Understanding of Chemical Reaction." International Journal of Science Education 20(3): 305-316.

Arnold, B. (1983). "Beware the molecell. Aberdeen College of Education. Biology Newsletter 42, 2-6." Aberdeen College of Education Biology Newsletter 42: 2-6.

Bloom, P. (2002). How children learn the meaning of words. . Cambridge, MIT Press.

Bodner, George M. (1991). I have found you an argument: the conceptual knowledge of beginning chemistry graduate students, Journal of Chemical Education 68 (5), 385-388.

Boo, H.-K. \& Watson, J. R. (2000). Progression in high school students' (aged 16-18) conceptualizations about chemical reactions in solution. Science Education, 85, 568-585. Chemical Reactions, Journal of Research in Science Teaching 35 (5), $569-581$.

Cakmaki, G., Donnelly, J., \& Leach, J. (August, 2003.). A cross-sectional study of the understanding of the relationships between concentration and reaction rate among Turkish secondary and undergraduate students. Paper presented at the European Science Education Research Association (ESERA) conference, Noordwijkerhout, The Netherlands.

Çalik, M. \& Ayas, A. (2005) A comparison of level of understanding of eighth-grade students and science student teachers related to selected chemistry concepts. Journal of Research in Science Teaching. 42(6), 638-667.

Carey, S. (1991). Knowledge acquisition: Enrichment or conceptual change? The epigenesis of mind (pp. 257-291). S. Carey and R. Gelman. Hillsdale, Erlbaum.

Cokelez, A., Dumon, A., \& Taber, K. (2008). Upper Secondary French Students, Chemical Transformations and the "Register of Models": A cross-sectional study. International Journal of Science Education, 30(6), 807-836.

Delgado, C. (2009). Development of a research-based learning progression for middle school through undergraduate students' conceptual understanding of size and scale. Ann Arbor, Univeristy of Michigan. Doctoral Disseration. 


\section{Structure, Properties, and Transformation of Matter}

Devetaka, I. \& S. Glazara (2010). "The influence of 16 year old students gender, mental abilities, and motivation on their reading and drawinng submicrorepresentations achievement." International Journal of Science Education 32 (12): 1561-1593

Dickinson, D. K. (1987). "The development of material kind." Science Education 71: 615-628.

Driver, R. (1994). Making sense of secondary science : research into children's ideas. London ; New York, Routledge.

Driver, R., E. Guesne, et al. (1985). Children's ideas in science. Milton Keynes Buckinghamshire ; Philadelphia, Open University Press.

Freyberg, P. \& R. Osborn (1985). Learning in science: the implications of children's science. Portsmouth, NH, Heinemann.

Gensler, W. (1970). Physical versus chemical change. Journal of Chemical Education 47

(2), 154-155.

Griffiths, A. K., \& Preston, K. R. (1992). Grade-12 students' misconceptions relating to fundamental characteristics of atoms and molecules. Journal for Research in Science Teaching. 29(6), 611-628.

Hesse, J. J. and C. W. Anderson (1992). "STUDENTS CONCEPTIONS OF CHEMICAL-CHANGE." Journal of Research in Science Teaching 29(3): 277-299.

Horton, C. (2007). Student alternative conceptions in chemistry. California Journal of Science Education, VII(2).

Kesidou, S., Duit, R. \& Glynn, S.M. (1995). Conceptual Development in Physics: Students' Understanding of Heat; In S.M. Glynn and R. Duit (Eds), Learning Science in the Schools,(pp. 179-198). Mahwah NJ: Lawrence-Erlbaum

Kalkanis, G., Hadzidaki, P., \& Stavrou, D. (2003). An instructional model for a radical conceptual change towards quantum mechanics concepts. Science Education, 87, 257-280.

Lee, O., Eichinger, D.C., Anderson, C. W., Berkeimer, G.D. and Blakeslee, T.D. (1993). Changing middle school students' conceptions of matter and molecules, Journal of Chemical Education 67, 248-252.

Lee, O., D. C. Eichinger, et al. (1993). "Changing middle school students' conception of matter and molecules." Journal of Research in Science Teaching 30(3): 249-270. 


\section{Structure, Properties, and Transformation of Matter}

Johnson, P. (1998). "Progression in children's understanding of a 'basic' particle theory: a longitudinal study." International Journal of Science Education 20(4): 393-412.

Johnson, P. (2000). "Children's understanding of substances, part 1: recognizing chemical change." International Journal of Science Education 22(7): 719-737.

Kind, V. (2004). Beyond Appearances: Students' misconceptions about basic chemical : 2nd edition, Durham University.

Krajcik, J., K. L. McNeill, et al. (2008). "Learning-goals-driven design model: Developing curriculum materials that align with national standards and incorporate project-based pedagogy." Science Education 92(1): 1-32.

Merritt, J. (2010). Tracking Students' Understanding of the Particle Nature of Matter. Ann Arbor, University of Michigan. Doctoral Dissertation.

Mohan, L., J. Chen, et al. (2009). "Developing a multi-year learning progression for carbon cycling in socio-ecological systems." Journal of Research in Science Teaching 46(6): 675-698.

Mulford, Douglas R. (1996). An Inventory for Measuring College Students' Level of Misconceptions in First Semester Chemistry. Master of Science Thesis, Purdue University.

Nakhleh, M. B. \& A. Samarapungavan (1999). "Elementary school children's beliefs about matter." Journal of Research in Science Teaching 36(7): 777-805.

Novick, S. \& J. Nussbaum (1978). "Junior high school pupils' understanding of the particulate nature of matter: An interview study." Science Education 62(3): 273-281.

Osborne, Roger J. \& Cosgrove, M.M. (1983). Childrens' conceptions of the changes of state in water, Journal of Research in Science Teaching 20, 825-838.

Papageorgiou, G and Johnson, P (2005). Do Particle Ideas Help or Hinder

Pupils' Understanding of Phenomena? International Journal of Science Education 27(11)1299-1317.

Pallant, A. \& Tiner, R. (2004) Reasoning with Atomic-Scale Molecular Dynamic Models. Journal of Science Education and Technology $13(1): 51-66$

Renström, L., B. Andersson, et al. (1990). "Students' conceptions of matter. ." Journal of Educational Psychology 82(3): 555-569. 


\section{Structure, Properties, and Transformation of Matter}

Smith, C., J. Snir, et al. (1992). "Using conceptual models to facilitate conceptual change: The case of weight/density differentiation." Cognition \& Instruction 9(3): 221-283.

Schmidt, Hans-Jurgen (1997). Students' Misconceptions - Looking for a Pattern, Science Education 81 (2), $123-135$.

Smith, C. L., M. Wiser, et al. (2006). "Implications of Research on Children's Learning for Standards and Assessment: A Proposed Learning Progression for Matter and the Atomic-Molecular Theory." Measurement: Interdisciplinary Research and Perspectives 4(1-2): 1-98.

Smith, C. L., M. Wiser, et al. (2011). Abstracting a General Concept of Matter among Grade 3-5 Students: Lessons from the Inquiry. Annual Meeting of the Jean Piaget Society. Berkeley.

Stavy, R. (1991). "Children's ideas about matter." School Science and Curriculum 91: 240-244.

Stevens, S. Y., C. Delgado, et al. (2010). "Developing a Hypothetical Multi-Dimensional Learning Progression for the Nature of Matter." Journal of Research in Science Teaching 47(6): 687-715.

Treagust, D.F., Chittleborough, G, Mamiala, T.L. (2003). International Journal of Science Education 25(11): 1353-1368)

Taber, K. S., \& Coll, R. K. (2002). Bonding. In Gilbert, J. K., Jong, O. D., Justi, R., Treagust, D. F. \& Van Driel, J. H. (Eds.), Chemical Education: Towards Research-Based Practice (pp. 213-234). Dordrecht, Netherlands: Kluwer Academic Publishers

TERC. (2011). "The Inquiry Project: Bridging Research and Practice." from http://inquiryproject.terc.edu/.

Unal, R., \& Zollman, D. (1999). Students' description of an atom: a phenomenographic analysis. Downloaded from http://perg.phys.ksu.edu/papers/vqm/AtomModels.pdf

Wiser, M., K. O'Conner, et al. (1995). Mutual Constraints in the Development of the Concepts of Matter and Molecule. American Educational Research Association (AERA). San Francisco, CA.

Wiser, M. \& C. L. Smith (2008). Learning and Teaching about Matter in Grades K-8: When Should the Atomic-Molecular Theory Be Introduced? International Hanbook of Research on Conceptual Change. S. Vosniadou. New York, Routledge.

Wiser, M., C. L. Smith, et al. (In Press). Learning Progressions as tool for curriculum development: Lessons from the Inquiry Project. Learning Progression in Science. A. Alonzo and A. Gotwals, Sense Publishing.

Van Driel, J. H. \& Gräber, W. (2002). The teaching and learning of chemical equilibrium. In Gilbert, J. K., Jong, O. D., Justi, R., Treagust, D. F. \& Van Driel, J. H. (Eds.), Chemical Education: Towards Research-Based Practice (pp. 271-292). Dordrecht, Netherlands: Kluwer Academic Publishers 


\section{Narrative: \\ The Hypothetical Learning Progression for The Flow of Matter \& Energy in Ecosystems}

The purpose of this narrative is to provide an overview of the hypothetical learning progression for the Flow of Matter \& Energy in Ecosystems and how it was developed. We address which core concepts from the National Research Council's Framework for $\mathrm{K}-12$ Science Education align with this learning progression and the reasons why we structured the table as we did. Additionally, we summarize three key aspects of the learning progression that are presented in the table representation:

o the key features of students' explanations or explanatory models, and changes in those explanations from level to level,

o useful or productive ideas and experiences that may serve as leverage from one level of understanding to another, and

0 trends and changes in learning performances and experiences across the levels.

At the end of this narrative we provide the reader with an annotated outline of these three features.

In the section that follows we provide more details about how and why we developed each part of the hypothetical learning progression (HLP) table, including the research used to develop and inform the HLP, and in the second section, we provide the storyline of the table.

\section{Developing the HLP}

The development of the hypothetical learning progression for the Flow of Matter and Energy in Ecosystems was stimulated by the Framework for K-12 Science Education developed by the National Research Council (National Research Council, 2011). In order to fit the research evidence about how students progress through successively more sophisticated levels of understanding of phenomena in living systems involving the flow of matter and energy and the concepts identified in the NRC framework we had to combine some of their core concepts (specifically concepts from LS2.B, but also pieces from LS1.A and LS1.C). While the way we organize the concepts in this 
learning progression is somewhat different from the NRC framework or past standards documents, a close comparison of the NRC framework with our progression reveals that most of the ideas found in this progression are also present in the NRC framework, albeit with some elaborations and a few small additions. This difference in organization reflects a desire to represent in the progression the coherence we see in the explanations that students are likely to offer at different levels. Appendix $F$ shows this relationship between the NRC framework and the explanations or models we identified in the table of the HLP.

\section{The Research}

Several types of research provide the foundation for the HLP. Most directly helpful is empirical research that documents how students' ideas develop over time, how students demonstrate and apply their knowledge, and what experiences or ideas might support the development of new understandings. Work of this sort sometimes is done in the context of designing instructional interventions to support the development of students' thinking and understanding, with close attention being paid to how their thinking and understanding in fact develop in response. Other studies track students' thinking as it develops in response to the instructional experiences schools provide. The work of Mohan, et al. (2009), upon which we drew heavily, falls in this latter category. Research by Lehrer \& Schauble (in press; 2000), which we also drew upon, is of the former type. These studies show that with appropriate scaffolding for the target knowledge and practices, learners can engage in scientific practices and develop versions of concepts that can be very powerful, such as reasoning about growth of plant or animal populations or engaging in scientific investigations earlier than is commonly targeted in current standards and benchmarks.

\section{Student Explanations in the HLP}

Our HLP identifies the conceptual models or explanations that, with instruction, students are likely to develop and apply to understand natural phenomena-in this case students' explanations for phenomena related to the flow of matter and energy in ecosystems. Such natural phenomena include, for example, that the growth and functioning of all organisms is dependent on food; the growth and functioning of plants are dependent on carbon dioxide in the atmosphere, and the carbon atoms present in plants or animals came from other organisms, etc.

Our team of consultants considered the most current research in the area (Mohan, Chen, et al., 2009; Lehrer \& Schauble, 2000; Lehrer \& Schauble, in press) to identify the 
types of explanations students might develop during their school careers. The organization of the explanations at each level of the table reflects these research findings and tracks changes in students' understanding of the flow of matter and energy in ecosystems by considering three key questions that their explanations or models might address: 1) what do organisms need to grow and survive?, 2) what do organisms do with things they get from their environments?, and 3) how are organisms connected to each other and their environments? (informed by Mohan et al., 2009).

\section{Selection of Learning Performances}

The HLP for the Flow of Matter and Energy in Ecosystems includes examples of the kinds of learning performances that students at each level are likely to be capable of demonstrating and, as we stressed in the Preface, the kinds of learning experiences that would support students in advancing through these levels. We have developed schemes for systematically designing learning performances and clustering science practices to ensure all the practices are represented in the set of learning performances (see Appendix B). We have applied this design and clustering scheme to generate the set of learning performance examples shown in our HLP. This is only a sample of the performances that might be imagined, but it provides an appreciation of the nature and level of sophistication of what students might be able to do when they have reached the level being described. Again, as noted in the Preface, students' sophistication in the application of scientific practices can also be described in learning progression terms, and the learning performances included in the HLP reflect an interaction between their understanding of the relevant concepts and their application of the practices. Ideally the selection of learning performances should be based on knowledge of the kinds of experiences students have been exposed to during instruction, and the criteria for judging their performances on both concepts and practices should reflect the levels that those instructional experiences have exposed them to, and have expected from them.

\section{The HLP Storyline}

An overview of the storyline of the HLP is provided below and can be used in conjunction with the table to build a full picture of the progression. 
At the largest grain size, the HLP for the Flow of Matter and Energy through Ecosystems describes four levels of explanatory models that students are likely to move through in an ordered sequence:

o A simple needs-based explanation in which living things "need" things from their environment to grow and live like food and water and there is little awareness of the specific substances (certainly not matter in a scientific sense) required to support organism growth and function. There is little or no ability to trace the flow of matter between or within organisms.

- A materials-based explanation in which living things require specific materials [e.g. food and water for animals and access to air and water for plants] from their environment to grow and live; students are more likely to differentiate food as a material that is needed as opposed to more general needs for survival like shelter. This is a precursor to an ability to trace matter within organisms (i.e. there is a notion that animals somehow do things with food after they ingest it even though students at this level are very unclear about the mechanisms).

- A substance-based explanation in which living things require specific substances (e.g. sugars found in foodstuff) to support growth and function. Students begin to show an ability to trace the flow of matter between organisms through food chains and food webs and a better understanding of the internal mechanisms that transform and transport molecules derived from food to other parts of the body to support growth and function. There are also emerging understandings that foods are made of molecules and those molecules are transformed to other molecules.

- An atomic-based explanation in which living things require specific elements (namely carbon) found in specific compounds to support growth and function. There is greater ability to trace the flow of matter between organisms because there is greater ability to understand the chemical and cellular processes involved in the transformation and movement of organic and inorganic matter within and between organisms that can be applied to all organisms in an ecosystem [including plants, animals, and decomposers]. Principles of conservation of matter and conservation of energy also are applied to further allow students to trace the flow of matter and energy. 
These four levels represent different conceptual models that students may develop, but they do not develop without support from teachers or curriculum materials providing students with learning experiences that expose them to the relevant phenomena and require them to use scientific practices in ways that will prepare them to demonstrate the kinds of learning performances expected at each level. A key task for students is to reflect on their knowledge of how living organisms grow and function and how they are dependent on each other and the environment. Students should be testing their assumptions using their observations and other new evidence to revise their explanations. Teachers have an important role in providing feedback on how well students' explanations account for and predict the phenomena of interest, and what they cannot explain or predict well. With such support, students will be able to revise their conceptions in ways that reflect the progression we are describing.

\section{Useful or Productive Ideas}

A learning progression perspective is consistent with a developmental perspective on learning in that both emphasize that students learn by integrating new ideas with preexisting ideas. It therefore attempts to identify the ideas students initially hold (perhaps incomplete or partially incorrect ones) so that students and teachers can build on them to develop new conceptions. In light of this, the HLP developed for the flow of matter and energy in living systems identifies useful or productive ideas or experiences at each level that might serve as leverage for moving students to another level.

The outline below highlights the key features of the explanations students are likely to offer at each level in the progression as well as some of the useful or productive ideas or experiences at each level that might support consolidating their explanations at that level or moving to the next one. It includes notes on the trends found in the sample learning performances and experiences across the levels.

Without going into great detail, we point out a few ideas that, while they are "wrong" or incomplete in some sense, may nevertheless serve as levers or stepping stones to developing more sophisticated models of the flow of matter and energy. At level 2 students may understand that animals ingest food and somehow do something with that food inside of their bodies because the more food they consume the more they grow. While incomplete, this idea is still useful because it is a precursor to the ability to trace the flow of matter within organisms, and this is a key understanding needed in order to trace the flow of matter through an ecosystem. These early notions are about mechanisms hidden inside of organisms that students infer must be there, but they are 
unclear what they are [for example, perhaps students think that animals have internal organ systems to deal with food but have incomplete ideas about how exactly these organs relate to the flow of materials inside of an organism] .

At level 3 these initial ideas about hidden internal mechanisms can be elaborated upon to develop more sophisticated models of how food is transformed and transported within organisms to support their growth and functioning. In another example useful or productive ideas can be found by comparing explanations and student ideas at level 3 and 4. At level 3 the notion that specific compounds like sugars are found in food and that food is made of molecules-as well the idea that cells in organisms are made of molecules, can leverage the development of a more complete picture at level 4 of how atoms are rearranged during chemical and cellular processes to support an organism's growth by incorporating carbon containing compounds into the organism's cellular structures.

\section{Learning Performances and Experiences}

A review of the learning performances and experiences for the HLP for the flow of matter and energy in ecosystems reveals some important changes in students' thinking across the grades. Students' explanations and arguments draw upon increasingly sophisticated models of matter and the processes involved in transforming matter (see the HLP for the structure, properties, and transformations of matter) as well as more sophisticated evidence about the relationships between matter and changes in growth or function. These changes are most evident in the criteria associated with learning performances organized under cluster 1 (explaining, predicting, and developing explanatory models) and cluster 2 (constructing scientific arguments) For example, at level 2 a learning performance asks students to construct a model for plant growth that accounts for differences in availability of resources, and a similar performance is found at level 3. At level 2 the criteria reflect the simple explanation that specific materials are needed to support plant growth like air and water for plants. However the criteria for level 3 ask students to model how mass is transferred between organisms and to include more specific substances, in particular carbon dioxide, and to exclude water or soil since they are not incorporated in the biomass of a plant. This contrast in the criteria reveals that at level 3 students are being asked to draw upon more refined substancebased explanations for matter needed to support growth as well as more specific notions of what matter is incorporated into plant structures. Of course their ability to perform according to these criteria will depend on their exposure to experiences that give them the opportunity to attend to the relevant phenomena and to engage in the practices that would help them develop and understand the explanations for them. 
The sample learning performances also reflect students' increasingly sophisticated application of ideas as reflected in their opportunities to design investigations or analyze data that involve more complex forms of evidence. For example, at level 1 students' understanding of what counts as growth may be limited so they will focus on easily visible changes such as body length, even though that might not be the best measurement to relate consumption of food to growth. However, at levels 2 and 3 students develop better understandings of what makes up food and then may realize there are other ways to measure growth and ultimately, at level 3, students may realize they must consider change in mass of the organism as it relates to the absorption or ingestion of carbon matter by plants or animals.

For another example of how learning experiences and performances encourage and reveal changes in students' ability to apply their explanations and models in increasingly more sophisticated ways, consider the cluster 2 practices at level 3 and 4 . At level 2 students conduct investigations or analyze data sets that relate the change due to ingestion or absorption of matter by organisms to change the mass of the organism, but at level 3 students engage in the analysis of radio labeled carbon and identify patterns in which the carbon is found or accumulated as part of a virtual data set. In this example, students at level 4 have more sophisticated atomic-molecular models to draw upon and a better ability to trace matter so they can apply that knowledge to understand and interpret the results of radio-labeled carbon experiments. In contrast at level 3, students have less developed notions of matter and the processes involved so they would focus on more macroscopic forms of evidence such as weight changes.

\section{Outline of the HLP}

Since the storyline of the HLP for the flow of matter and energy in ecosystems may be difficult to follow in the table, the following outline presents it in a more simplified form. This outline highlights the key features of the explanatory model applied at each level, some key ideas within each model that might serve as leverage to the next level, and some notes on how ideas are being used in more sophisticated ways as reflected in the learning experiences and performances. While the outline highlights key differences across the levels of the HLP, the table representation also includes sections that highlight "what is changing in students' thinking" and that section provides more complete descriptions of the changes between adjacent levels.

\section{Level 1: Simple need-based explanations}

o Key features of student explanations: 
- What do organisms need to grow and survive?: Students' explanations are in terms of simple needs (i.e. animals or plants "need" certain things from their environment to grow and live-these things are viewed as "enablers" necessary growth or to stay alive) but there is little awareness of the specific substances required for living organisms to grow or survive (nor are energy requirements mentioned). One shift from Pre-K is to broaden students' conception of what is alive and the criteria by which they define "living."

- What do living things do with things they get from their environment?: Students have little awareness of what organisms do with the things obtained from the environment (but may have a sense they do something).

- How are organisms connected to their environment?: Students focus on simple relationships between animals and plants (like animals eat plants).

- Students' ability to connect growth with consumption of food is a precursor to their ability to trace matter between plants and animals.

- Students' ability to recognize that organisms' need to get things from the environment in order to grow and survive may leverage their thinking about the need for specific substances to support growth and survival.

o Learning performances:

- In constructing explanations students draw upon simple notions of "enablers" to support growth and functioning; they have little notion that matter is critical to supporting growth because they may not realize air or water is matter. Tthey identify what animals need as the action of eating other living or dead things (plants or animals).

- In constructing explanations and arguments students show little ability to trace matter within organisms and have no mechanisms in mind for this, but begin to see simple relationships between food taken in and visible macroscopic changes in growth (i.e. when food is consumed the organism grows).

- In deigning investigations students identify tangible and visible features of growth that relate to consumption of food: they identify a positive correlation between food taken in and growth.

\section{Level 2: Materials-based explanations}

o Key features of student explanations:

- What do organisms need to grow and survive?: Students explanations begin to focus on the specific materials needed from the environment such as food or water for plants and animals to grow; students begin to recognize food ingestion in animals is a key factor in their growth. Decomposers are recognized as living. The needs of organisms and the ways that organisms satisfy those needs become more differentiated. At this level, while the focus may still be on cause and effect (a caterpillar needs food in order to grow and stay healthy, but they are more narrowly focused on material needs as well as prior actions (e.g. a caterpillar eats food in order to grow). 
- What do organisms do with things they get from their environment?: Students begin to recognize that something happens to food once it is consumed by organisms, and it somehow supports growth. .

- How are organisms connected to their environment?: Students connect animals to plants because animals eat plants; plants need sunlight, air, and water in their environment [before connections are just limited to plants and animals].

- Students' recognition that both plants and animals are growing and that animals take in food, whereas plants do not [students need to see plants and making their own food at the next level].

- Students' understandings about the needs of plants and animals represented as a web provide a basis for later understanding of simple cycles such as animals eat plants, animals decompose, and those material "cycle" back to plants.

- Students' recognition that animals do something with food once ingested is a precursor to an idea that matter can be traced inside of organisms, even though students might have unclear ideas of how food is digested and distributed inside organisms.

- Students' realization that there are more ways to measure intake of food as it relates to changes in growth [ultimately students need to think about changes in weight as a function of growth].

o Learning performances:

- In constructing explanations students identify specific materials (e.g. water, air food)

- In constructing explanations, designs, and arguments students show a beginning ability to trace matter within organisms - although mechanisms are unclear; this recognition is evident in arguments where the student can recognize co-variation in food consumed and growth (now realize more food results in more growth)

- In designing investigations of growth students use multiple measures of growth such as height, weight, reproductive capacity. If they examine weight, students may now realize that there are instruments that provide more accurate and sensitive measures of growth [before students would be more likely to rely on human senses to detect change]. Thus notions of what counts as evidence for measures of growth is becoming more sophisticated.

\section{Level 3: Substance-based explanations}

o Key features of student explanations:

- What do organisms need to grow and survive?: Focus on specific substances found in food as sources of building material and energy (e.g. sugar); here the energy story emerges and students recognize that energy is something needed to support organism growth and function.

- What do organisms do with things they get from their environment?: Begin to recognize chemical transformations of matter occur inside of plants and animals to support their growth and functioning; students develop a better model of the internal mechanisms involved in the flow of matter inside of plants and animals].

- How are organisms connected to their environment?: Explanations recognize more interdependent connections among plants, animals, and decomposers in influencing 
the flow of matter in ecosystems [see connection in terms of food chains and food webs and can recognize that plants may get something from animals-- e.g. carbon dioxide).

o Ideas of leverage to next level:

- Ideas that allow students to trace the flow of matter and energy are useful here, including a more complete explanation for how matter is broken down inside of organisms and distributed within them, as well the ideas that cells are composed of matter and that organism growth is a process that involves successively more cell reproduction.

- Students should be able to explain that organic matter includes both simple and complex molecules containing $\mathrm{C}, \mathrm{H}, \mathrm{O}$ [it would be good for students to realize also that cells also are composed of similar molecules with these same substances, but that might not develop until high school].

- Students should be able to distinguish between organic matter which has stored chemical energy that living organisms can utilize and other compounds which cannot be utilized as sources of chemical energy ${ }^{2}$, however, they do not need more elaborate analyses of types of organic matter. Identifying forms of energy such as light, heat, chemical energy and motion/metabolic functioning-is sufficient at this level and may leverage other ideas about energy later.

- In constructing explanations or arguments students draw upon understandings that food substances are made of molecules and rudimentary understandings that matter is transformed as a result of chemical reactions and that these processes occur inside of cells.

- In constructing explanations or arguments students draw upon an emerging understanding of energy and identify energy sources and some basic energy transfer events so they can begin to trace energy [before explanations do not include or account for energy].

- In constructing explanations, designs, and arguments students show a beginning ability to trace the flow of matter within and between organisms and between the environment and organisms: this ability is reflected in students' more sophisticated explanations of what organisms do with matter and how they do it.

- In designing investigations, students increasingly focus on intake of specific materials and changes in biomass [at this levels students' designs show an increasing sophistication in measuring amounts of living matter (dry mass) and continuity of material kinds between food sources and living organisms].

\section{Level 4: Atomic-based Explanations}

o Key features of student explanations:

- What do organisms need to grow and survive?: Continued refinement of what organisms need to support growth by recognizing the central role of carbon in matter

\footnotetext{
${ }^{2}$ This is a deliberate oversimplification. We recognize that some organisms utilize other sources of chemical potential energy that are inorganic matter, but research on student learning in this area suggests that this oversimplification may still be useful for students when they are expected to think about bonds in compounds breaking and reforming during cellular respiration at the next level (C.W. Anderson, personal communication).
} 
taken in by organisms. Also recognize the similarity among most organisms in the cellular and atomic-molecular processes that govern growth and survival (such as digestion and biosynthesis) as well as the unique and important role for the photosynthetic processes.

- What do organisms do with things they get from their environment?: There is a refined understanding of the role of atomic-molecular mechanisms and cellular processes that support the flow of matter within organisms and between organisms. There is a better understanding of how food it utilized as a source of energy in living systems by recognizing the transfer of energy when the bonds of carbon-containing organic compounds are broken and the bonds of energy-carrying compounds are formed.

- How are organisms connected to their environment?: There is a refined understanding of how carbon flows in, and is cycled through, an ecosystem based on the interdependent relationships of the organisms in the ecosystem and how energy flows through and out of the system as a result. There is also a greater acknowledgment of the atomic-molecular processes and principles of energy transfer that govern the pattern of matter and energy flow in ecosystems.

o Learning performances and experiences

- In constructing explanations for growth and function students draw upon more refined understandings of matter and matter transformation by using the atomic-molecular model of matter, and they include more refined understandings of how energy is utilized from food stuffs. Principles of conservation of energy and matter are also applied here.

- In constructing explanations, designs, and arguments students have a more refined ability to trace matter and energy between organisms and between the environment and organisms: in particular, the ability to trace matter in explanations and investigations depends on their ability to recognize carbon as the key element that flows between organisms and between the environment and organisms.

- In analyzing data students are capable of interpreting and explaining more multifaceted data sets reflecting the flow of matter and energy in organisms and ecosystems [before notions of energy flow in living systems were just developing].

- In constructing explanations and arguments students also have a better account for how matter and energy flow through organisms and ecosystems as they develop better models for how energy is transferred.

- In constructing arguments students can draw upon more sophisticated models of matter and energy flow as well as a more refined understanding of the interconnections between and among organisms and between the environment and organisms to make sense of more complex phenomena such as perturbations in ecosystems that have multiple cascading effects on ecosystems. 
Figure 1. Snapshot of some of the different sections found in the table for this HLP

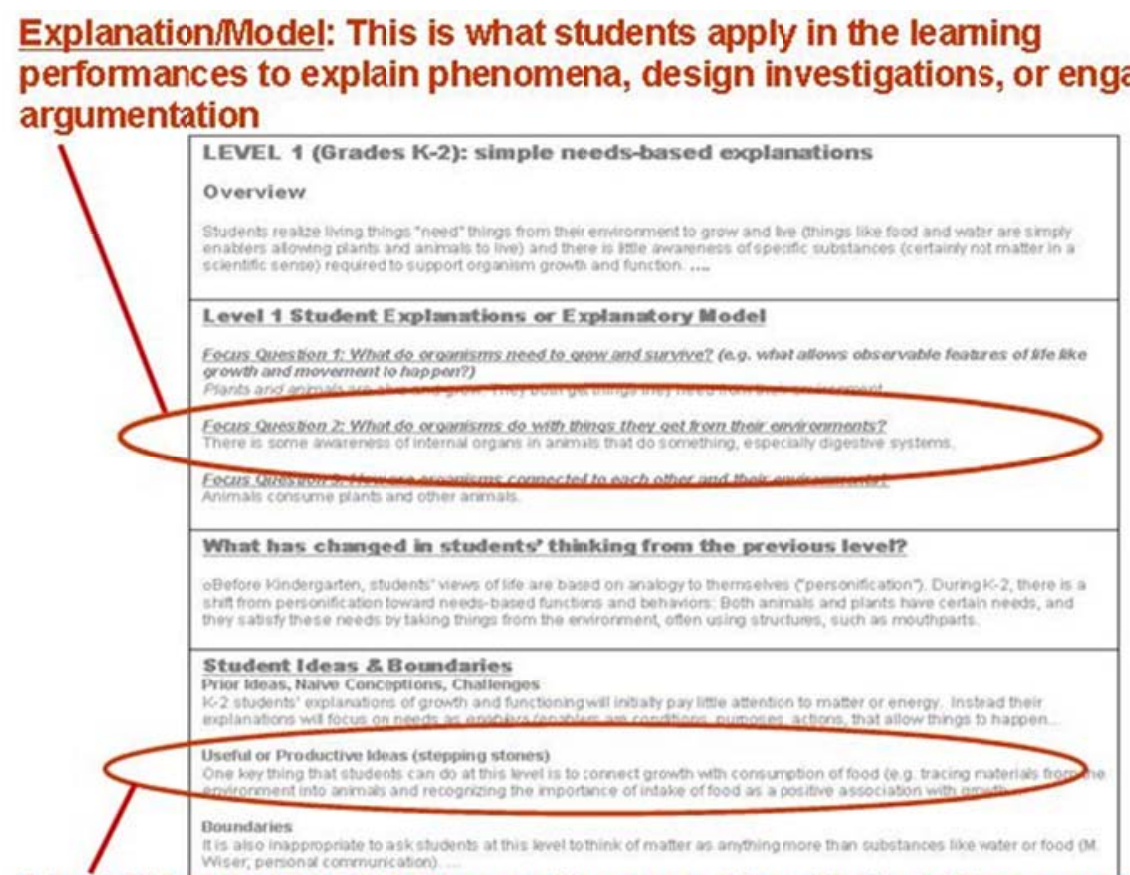

Useful Ideas: Ideas of leverage. These are ideas that might support development of ideas within a level or at the next level

\footnotetext{
Rationale and Notes

oWhille not part of the framework, it seems reasonable to assume students might have some notion of imtemal oryans Which ther can continue to think about into the nenstevel in erder to begin developirg a notion of interna mechaniams witrin orgarisms, therefore, their explanations should teitect this

How students demonstrate and apply their knowledge (as reflected in Learning Performances)

oln censtructing explanations students shoud drawupon smple notions that "enablers" support growth ard functioning, ther have litte notion of matter as a constituent of a nenibler. In this view iling things smply have a tendency lo engage in

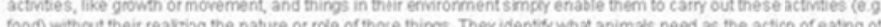
food) without their reaising the nature or role of those things. They identify what animals need as the acticn of eating other things, living or dead foumts or antmaki

oln constructingexplanations and araments students wall lkely show itwe abiliy to trace matter within organisms and have thapess

Sample Learning Experiences \& Learning Performances (LE/.P)

Explaining aredicting, feveloping explanatory nodets (cluster 1 )

LERP F constructan onp anaton of how an animal gets what a needs to survire

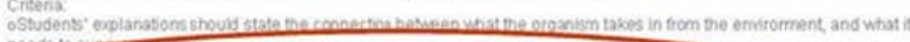

Designing imvestigations, collecting, representing, and analvaing data (cluster 2 )

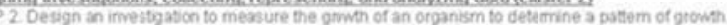
Critaris c

Cobjents' imestigasons of growth should include the specification of a massue of a macioscopic

Learning performances: These refect how knowledge is applied in context of specific science practices. These performances grow in sophistication as explanations or models change
} 


\section{References}

Mohan, L., J. Chen, et al. (2009). "Developing a multi-year learning progression for carbon cycling in socio-ecological systems." Journal of Research in Science Teaching 46(6): 675-698.

Lehrer, R., Schauble, L., Carpenter, S., \& Penner, D. (2000). Designing classrooms that support inquiry. In J. Minstrell \& E. V. Zee (Eds.), Inquiring into inquiry learning and teaching in science (pp. 80-99). Washington, DC: American Association for the Advancement of Science.

Lehrer, R., \& Schauble, L. (in press). Seeding evolutionary thinking by modeling its foundations. Science Education.

National Research Council (2011). A Framework for K-12 Science Education: Practices, Crosscutting Concepts, and Core Ideas. Washington D.C.: National Academies Press. 


\section{Flow of Matter \& Energy in Ecosystems: Level 1}

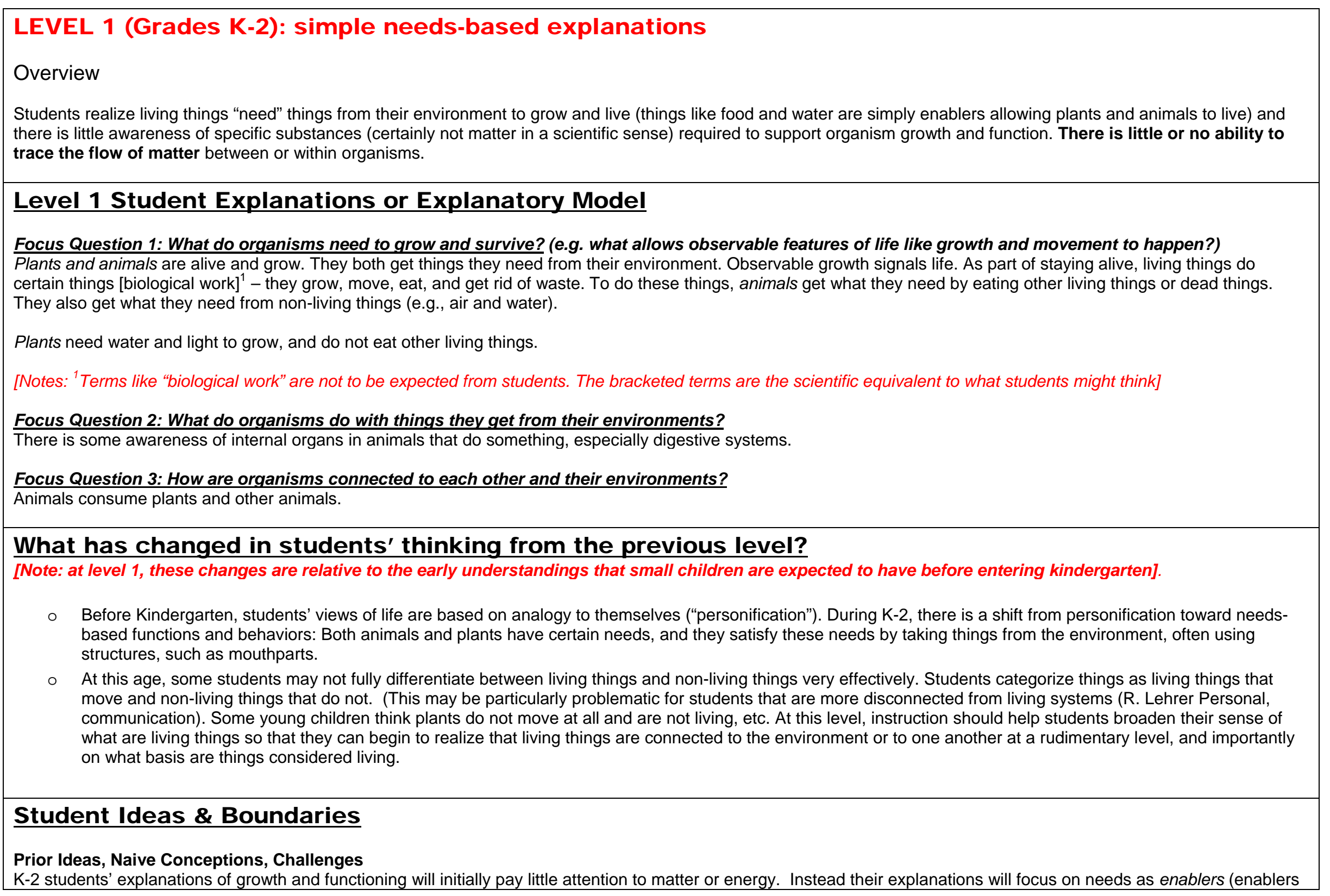




\section{Flow of Matter \& Energy in Ecosystems: Level 1}

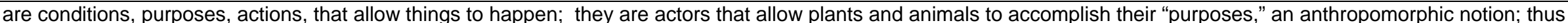
for example, plants would originally grow and water is just is one of those things a plant needs to accomplish this activity) (Mohan, Chen, et al, 2009; Lehrer \& Schauble, 2000).

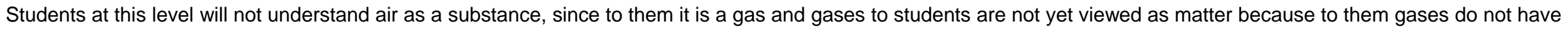
weight and cannot be felt (Wiser and Smith, 2008).

Students at this level do not think of decay of organic matter as involving microscopic organisms. Either they think of mechanical processes, such as breaking and

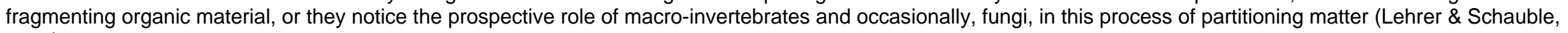
2000).

Students at this level will have little understanding of the mechanisms for what organisms do with matter once eaten (Mohan, Chen et al, 2009), although they may have some awareness that organism do something with food

\section{Useful or Productive Ideas (stepping stones)}

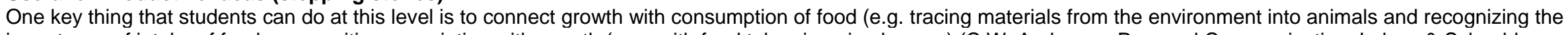
importance of intake of food as a positive association with growth (e.g., with food taken in animals grow) (C.W. Anderson, Personal Communication; Lehrer \& Schauble, 2000a,b, Lehrer \& Schauble, in press).

The ability to recognize that organisms need to get things from the environment in order to grow and survive may be leverage to thinking about the need for specific substances to support growth.

The ability to develop and employ measures that indicate macroscopic growth is an important resource for beginning to trace the flow of matter.

\section{Boundaries}

It is also inappropriate to ask students at this level to think of matter as anything more than substances like water or food (M. Wiser; personal communication).

Children are not thinking about organisms as being in a system. Their view is one dominated by the organism (R. Lehrer, Personal communication; Lehrer \& Schauble, 2000a,b).

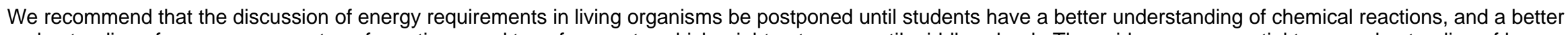

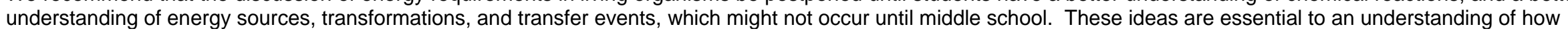
energy is utilized in living organisms.

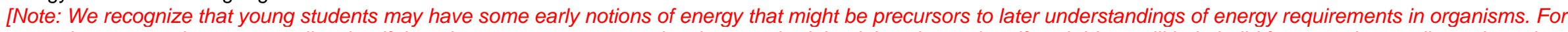

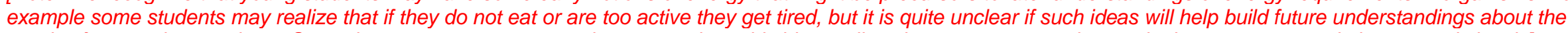
needs of energy in organisms. So we have suggest a conservative approach on this idea until we have more research to make better recommendations at early levels]

\section{Rationale and Notes}

o While not part of the framework, it seems reasonable to assume students might have some notion of internal organs which they can continue to think about into the next level in order to begin developing a notion of internal mechanisms within organisms, therefore, their explanations should reflect this.

$0 \quad$ In explaining what living things need, $\mathrm{K}-2$ students use personification as a resource to consider living things as having needs, initially by extension to familiar animals, such as pets, and later (by end of this grand band) including less familiar animals and plants, so their explanations at this level should focus on familiar organisms and familiar functions. 


\section{Flow of Matter \& Energy in Ecosystems: Level 1}

o We purposely use the word "things" as opposed to "materials" that living organisms need to live and grow because students at this level are not likely to have a well formed concept of "material" (M. Wiser, personal communication) nor will they necessarily distinguish material "enablers" such as food from non-material "enablers" such as love or shelter (C. W. Anderson, personal communication).

o In learning performances that require students to ask questions and conduct investigations of animal and plant growth, students begin to establish an association between consumption of materials and changes that signal growth. For animals, they can readily establish an association between consumption of plant structures, such as leaves, and growth. For plants, the association is more general, with little or no account of the change in biomass that occurs during growth.

o Although learning performances should eventually focus on the measurement of the amount of substances taken relative to changes in growth (since this is evidence for the tracing of matter), students at this level must first focus on clearly visible and tangible changes in organisms, which in this case would be length of an organism rather than other more direct measures of growth such as weight. They must also work on developing measures of these tangible changes in growth and examine and identify associations between their measures of growth and other simple means of accounting for what organisms consume (such as counting the number of leaves a caterpillar eats).

\section{Notes on how students demonstrate and apply their knowledge (as reflected in Learning Performances)}

o In constructing explanations students should draw upon simple notions that "enablers" support growth and functioning; they have little notion of matter as a constituent of an enabler. In this view living things simply have a tendency to engage in activities, like growth or movement, and things in their environment simply enable them to carry out these activities (e.g. food) without their realizing the nature or role of those things. They identify what animals need as the action of eating other things, living or dead (plants or animals).

o In constructing explanations and arquments students will likely show little ability to trace matter within organisms and have no mechanisms in mind for this, but begin to see simple relationships between food taken in and visible macroscopic changes in growth (i.e., when food is consumed the organism grows).

o In designing investigations students' should be able to identify tangible and visible features of growth that relate to consumption of food: they identify a positive correlation between food taken in and growth

\section{Sample Learning Experiences \& Learning Performances (LE/LP)}

Explaining, predicting, developing explanatory models (cluster 1 )

LE/LP 1. Construct an explanation of how an animal gets what it needs to survive.

Criteria:

o Students' explanations should state the connection between what the organism takes in from the environment, and what it needs to survive.

o Students' explanations should identify how animals get what they need as the actions of eating other living or dead things (plants or animals).

o Students' explanations should identify growing, moving, eating, and getting rid of waste among what animals need to do to survive. Explanations do not distinguish matter and energy at this point.

\section{Designing investigations, collecting, representing, and analyzing data (cluster 2)}

LE/LP 2. Design an investigation to measure the growth of an organism to determine a pattern of growth. Criteria:

o Students' investigations of growth should include the specification of a measure of a macroscopic character of the organism, such as a plant's height or an insect larvae's length, and a means to record the changing values of a measure over time. At the youngest grade, the measure need not be a numerical quantity. Instead, a method that would allow direct comparison of change is acceptable, such as cutting a length of string to represent the height of a plant at a particular point in time.

LE/LP 3. Construct a representation of growth that depicts the change in a measure of a macroscopic attribute of an organism as a difference between successive 


\section{Flow of Matter \& Energy in Ecosystems: Level 1}

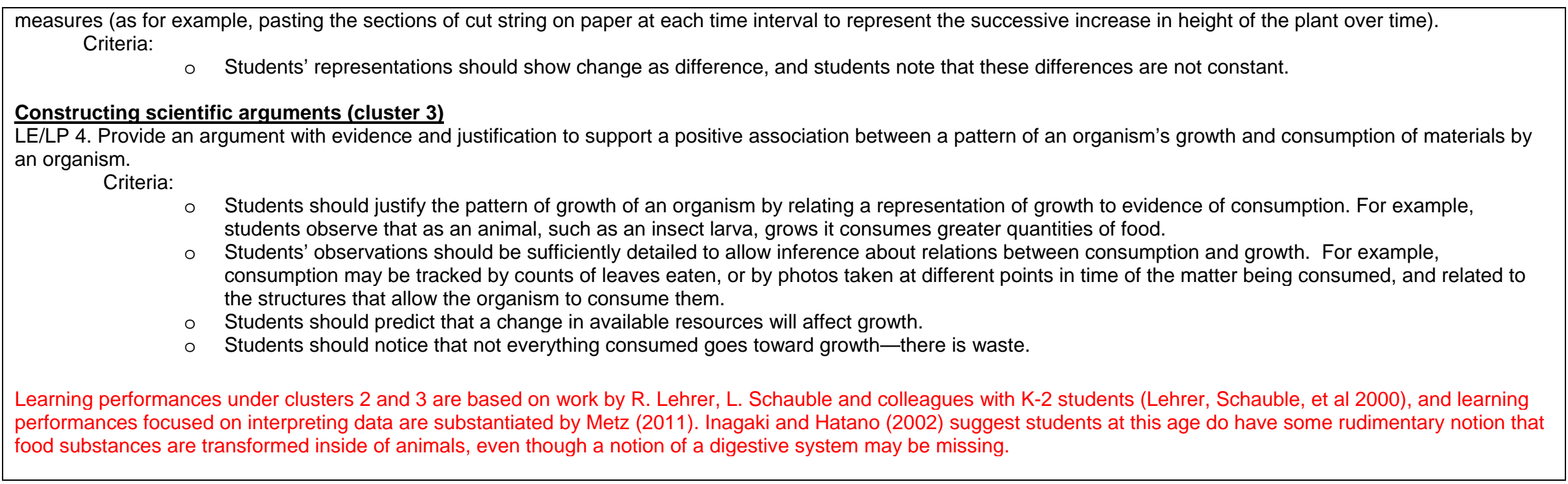




\section{Flow of Matter \& Energy in Ecosystems: Level 2}

\section{LEVEL 2 (Grades 3-5): Materials-based explanations}

\section{Overview}

Students realize living things require specific materials [e.g. food and water for animals and access to air and water for plants] from their environment to grow and live. Students are more likely to differentiate food as a material that is needed as opposed more general needs for survival like shelter. There is a precursor to an ability to trace matter within organisms (i.e. there is a notion that animals somehow do things with food after they ingest it even though students at this level are very unclear about the mechanisms)

\section{Level 2 Student Explanation or Explanatory Model}

\section{Focus Question 1: What do organisms need to grow and survive?}

Animals need to ingest food to grow [although these transformations are understood macroscopically and not at a cellular level. Animals also need water, air, and food in order to live, grow, and move. Water and air are not food.

Plants need water, air, and sunlight to live and grow.

Decomposers-macroscopic fungi and macroscopic invertebrates that facilitate decay-are alive and grow as well as plants and animals. Decomposers break down dead plants and animals and return materials (nutrients) that plants can use to the soil. [See notes under rationale for decisions made about decomposers in this explanation]

\section{Focus Question 2: What do organisms do with things they get from their environments?}

Plants and animals both move materials around inside their bodies. The material is used for growth and movement. In animals, some food moves through the digestive system and is eliminated as feces; other food reaches all parts of their bodies and is used for growth. Decomposers feed on dead organic material. [See notes under rationale for decisions made about the organ systems in this explanation]

\section{Focus Question 3: How are organisms connected to each other and their environments?}

All animals eat plants or other animals, so all animals are connected to plants. This connection is shown in a food web. Plants need sunlight for growth [even though sunlight is not food, its role in producing food and supporting growth is critical, so it may be okay if sunlight is part of a food web]. Decomposers are part of the food web too and feed off of dead plant or animal material.

\section{What has changed in students' thinking from the previous level?}

What do organisms need to grow and survive?

o Note that students at this level have not abandoned explanations that focus on conditions, enablers, purposes, actions, or results. At this level their explanations progress toward more general recognition that tracing matter can play an important role in describing and explaining growth, including:

1. recognition of decomposers as organisms that use dead plants and animals for food;

2. better ways of tracing matter qualitatively and quantitatively between organisms and their environments and among organisms (see notes on learning performances for this level) and;

3. increased ability to trace pathways of matter inside organisms

o At the previous level students focus on agency-cause and effect sequences involving actions of agents (e.g. a caterpillar has to eat so that it can grow and stay healthy), whereas at this level, while the focus may still be on cause and effect, students are more focused on material needs as well as prior actions (e.g. a caterpillar eats food in order to grow). 


\section{Flow of Matter \& Energy in Ecosystems: Level 2}

o The needs of organisms and the ways that organisms satisfy those needs each become more differentiated. The need for materials such as food may be furthe differentiated into types of food (e.g., some species thrive on milkweed in the larval stage). Students are similarly more attuned to the abiotic conditions needed to sustain and allow the organism to thrive (e.g., reproduce). For example, some species of plants require more light than others, so that one can predict their

abundance in some locations and not in others.

o The requirements for plant life and growth are now focused on specific factors: water, air and sunlight.

o Students' conception of what is living continues to broaden and now they are aware of decomposers as being living organisms also and can identify decomposers' needs for growth [dead plant or animal material].

\section{What do organisms do with things they get from their environments?}

o Students are now able to recognize the need for food (or other materials) to go to internal structures for delivery to other parts of the organism, whereas before there was no such mechanism [this represents a precursor for being able to trace matter within organisms].

\section{How are organisms connected to their environment?}

o Students now expand their understanding of the connections beyond the simple notion that animals eat plants, to include the understanding that plants need sunlight to grow. Therefore, the chain of connections in an ecosystem includes sun, plants, and animals. Decomposers are also introduced as having a role in the ecosystem in terms of restoring materials back to the soil.

\section{Student Ideas \& Boundaries}

Prior Ideas, Naive Conceptions, Challenges

Although students at this level should be able to describe relationships in food webs, they may view those relationships as simple cause-effect relationships (e.g. a bird consumes insects and grows) rather than seeing these relationships as descriptions of the flow and transformation of matter within a system (Mohan, Chen et al., 2009).

Students might think of the sun as food for plants (this idea might be okay at this level).

Students may still find it difficult to believe gases such as air or carbon dioxide have weight (Mohan et al. 2008; Wiser and Smith, 2008).

\section{Useful or Productive Ideas (stepping stones)}

Individual understandings about the needs of plants and animals being related in a web provide a basis for later understanding of simple "cycles" such as animals eat plants, animals die and decompose, and those materials "cycle" back to plants.

Understanding that animals do something with food once ingested is a precursor to the idea that matter can be traced inside of organisms, even though students might have unclear ideas of how food is digested and distributed inside organisms (C.W. Anderson, personal communication).

Identifying decomposers as living and growing [eventually students need to recognize these players in ecosystem as contributing to the flow of matter].

Recognizing more ways to measure the relationship between the intake of food and changes in growth [ultimately students need to think about changes in weight as a function of growth] as a qualitative trace of the flow of matter between organisms.

\section{Boundaries}

Students at this level do not need to explain functioning of organisms at the cellular or atomic-molecular scale. 


\title{
Flow of Matter \& Energy in Ecosystems: Level 2
}

\begin{abstract}
"Inputs" are not yet understood as atoms/molecules, and cells are not yet understood as the final destination for these "inputs."

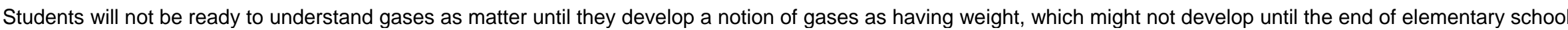
or early middle school (Wiser and Smith, 2008). Therefore, the idea that carbon dioxide is a gas that plants take and utilize to support growth (i.e., an increase in plant

mass) must wait until students have developed this understanding of the nature of matter.
\end{abstract}

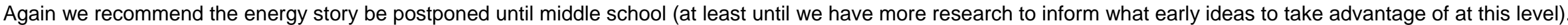

\section{Rationale and Notes}

o Ideas about the digestive and circulatory system in students' explanations (which were pulled from the core idea covering matter flow in organisms in early drafts of the NRC framework) seem appropriate at this level, because at the next level students need to connect ideas of how food is broken down by organisms to notions that building materials and energy storing molecules are distributed to other parts of the organisms to support growth and function. Therefore rudimentary ideas of matter transformation inside of organisms should begin at this level (Lehrer \& Schauble, 2004; in press). However, we do not extend mechanisms of digestion to other organisms like fungi because students need to build on their understanding of digestion in animals first and extend it to other organisms later on [our perspective is to build on students' intuitive ideas that organisms break down substances, which they likely understand in animals first, then they can apply the idea to plants and other organisms later on].

o We have introduced decomposers into students' explanations at this level because we think they are a vital part of the ecosystem and have an underappreciated role in the recycling of matter and therefore students should begin to think about decomposers earlier, at least as being living organisms. There is evidence to suggest students can think about decomposers in elementary school (Lehrer \& Schauble, 2000; Lehrer, Carpenter et al., 2000). However we do not suggest students at this level think about fungi on a cellular level because cells are part of students' explanations at the next level, level 3 . What is important is that students begin to focus on easily observable changes in organisms that signal growth has occurred in response to the presence of dead organisms (e.g. this might mean observing more worms growing in the presence of dead organisms or more fleshy mushrooms growing in the presence of dead organisms--again we are not focusing on cells that make up these organisms, but simply macroscopic changes).

o In one of the learning performances, we suggest that students should identify the co-variation, or relationship, between food consumed and amount of growth as evidence for the flow of matter within organisms. The amount of growth can include the height of plants or length of animals. Measures of growth of populations (such as reproductive capacity), as well as individual organisms, should be introduced as a stepping stone because ultimately students need to develop thinking about populations (R. Lehrer, unpublished research).

\section{Notes on how students demonstrate and apply their knowledge (as reflected in Learning Performances)}

o In constructing explanations, students are able to identify specific materials and their relative abundance as contributing to growth (e.g. water, air food) needed to support growth.

o In constructing explanations, designs, and arguments students show a beginning ability to trace matter within organisms - although mechanisms are unclear; this recognition is evident in arguments where one can recognize co-variation in food consumed and growth (now realize more food results in more growth).

0 In designing investigations of growth students include multiple measures of growth and now realize that instruments can provide more accurate and sensitive measures of growth [before students would be more likely to rely on human senses to detect change]. Thus, notions of what counts as evidence for measures of growth is becoming more sophisticated. 


\section{Flow of Matter \& Energy in Ecosystems: Level 2}

\section{Sample Learning Experiences \& Learning Performances (LE/LPS)}

\section{Explaining, predicting, developing explanatory models (cluster 1 )}

LE/LP 1. Construct a model of growth of a plant, animal, or decomposer that accounts for differences in growth as caused by differences in the availability of resources. [definitely must consider plants and animals]

Criteria:

o For plants, it is advisable to vary the availability of light and water so that student models can incorporate these fundamental resources into the model.

o For animals, the availability of resources such as water and perhaps the availability of preferred food sources (e.g., leaves that an insect prefers generally have more available energy to that insect than other species of leaves, even if the insect can consume them).

0 For decomposers, the rate of decomposition is influenced by types of food (i.e. type of organic matter present and whether it is living or dead), temperature, and the like. For students at the upper level of the grade band, growth should be represented by rate.

\section{Designing investigations, collecting, representing, and analyzing data (cluster 2)}

LE/LP 2. Design an investigation of the growth of an organism to determine how changes in resources (variations in conditions) affect growth.

Criteria:

o Students' investigations should include specification of multiple characters of an organism, such as a plant's height, size of the leaves, and the number of seeds it produces (e.g. reproductive capacity). The measures should result in quantities that are recorded at successive points in time. For chosen points of time, such as early and middle stages of growth, students should compare the measures from one or more organisms.

o Students should appreciate the value of data from multiple organisms. [Note: at later grades in this band, the measures include weight, so that students can begin to get a sense of biomass as an important measure of growth].

o If examining weight, students should recognize and employ instruments such as gram scales that may provide more accurate and sensitive measures of change in mass.

\section{Constructing scientific arguments (cluster 3)}

LE/LP 3. Provide an argument with evidence and justification to support a model that shows that some resource limits are more important than others in the growth of an organism. Criteria:

o Students should justify with supporting data that a resource may influence one aspect of growth more than another. For example, the height of a species of plant may not be affected by a change in the availability of light but other aspects of growth and reproduction will be (e.g., tall but spindly plants with few or no seed pods under conditions of low light vs. plants with greater number of leaves and many seed pods).

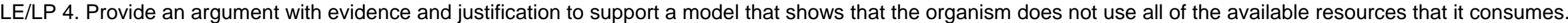
Criteria:

o Students compare the weight (mass) of what the organism consumes to the mass of the organism at multiple points of time. Then they should point out that the mass consumed is greater then the mass of the organism (this continues to deepen the sense of trophic levels and an orientation toward describing ecosystem functioning from the perspective of matter flow).

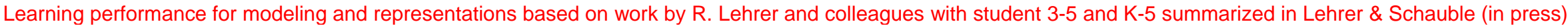




\title{
Flow of Matter \& Energy in Ecosystems: Level 3
}

\begin{abstract}
LEVEL 3 (Grades 6-8): Substance-based explanations
Overview

Students realize living things require specific substances (e.g. sugars found in foodstuff) to support growth and function. There is a beginning ability to trace the flow of matter between organisms through food chains and food webs and a better understanding of the internal mechanisms that transform and transport molecules derived from food to other parts of the body to support growth and function. There are also is an emerging understanding that foods are made of molecules and those molecules are transformed to other molecules. The energy story emerges in students' explanation at this level.
\end{abstract}

\section{Student Explanation or Explanatory Model}

\section{Focus Question 1: What do organisms need to grow and survive?}

Plants, animals, and decomposers obtain matter and energy that they need to live, function, and grow from their environments. Decomposers include microscopic bacteria and fungi as well as animal decomposers- all are characterized by using waste material from other organisms or dead matter as a source of food.

Animals and decomposers are different from plants in that they must consume food molecules (e.g. sugar) from other organisms (living or dead). Plants are unique in that they use the energy from sunlight to make sugars (food) from carbon dioxide and water. This process transforms light energy from the sun into stored chemical energy. These food molecules are used much the same way as in animals, they: a) react with oxygen to provide energy that is needed to carry out life functions, b) build and become incorporated into the body structure, or c) are stored for later use.

\section{Focus Question 2: What do organisms do with things they get from their environments?}

Plants, animals, and decomposers are all made of cells, which carry out life functions. Plant cells rearrange the atoms in carbon dioxide and water molecules and change them into sugar (food) and oxygen molecules. Sugars contain stored chemical energy. Minerals and other nutrients from the soil are not food (they don't provide energy), but plants combine them with sugar to make complex molecules that make up plant bodies and enable them to function. This is how plants grow.

Animals and decomposers grow by consuming complex molecules, distributing them to all parts of their bodies, and modifying them into other complex molecules. In animals food molecules are broken down in the digestive system and the resulting smaller molecules are transported to all the other cells of the body by the circulatory system where they can be incorporated into cell structures when growth occurs.

Inside the cells of plants, animals, and decomposers oxygen reacts with food (such as sugars) to produce carbon dioxide and water in a process called cellular respiration. Thus food molecules go through a transformation in which the atoms that make them up are rearranged to form new substances. When food reacts with oxygen it serves as a source of energy because the reaction with oxygen releases energy that the cell can use to drive growth or functioning (like movement in animals). This is energy originally obtained from sunlight and stored in the food compounds when they were produced by the plant.

Focus Question 3: How are organisms connected to each other and to their environments?

Matter: Matter is transferred between different organisms in an ecosystem. Plants get carbon dioxide from the atmosphere, which can come from 


\section{Flow of Matter \& Energy in Ecosystems: Level 3}

respiration carried out by all other organisms, including animals and decomposers, and transform this compound into food that plants can use [although certainly carbon dioxide from respiration is not the only means of introducing carbon dioxide into the atmosphere]. Animals get food from eating plants or other animals as described in food webs. Decomposers get food from dead animals or plants found in their environment and break down compounds found in this dead matter to generate freely available minerals (such as iron, magnesium, and other inorganic elements) that plants can absorb from the soil (and therefore help to recycle matter to plants).

Energy: Energy flows through ecosystems: sunlight is converted to chemical energy stored in organic, carbon-containing compounds, through photosynthesis in plants. That chemical energy is then stored in plant bodies and passed on to animals and decomposers. Plants, animals, and decomposers all use stored chemical energy for growth and other life functions; in particular animals use the chemical energy for necessary functions such as movement.

\section{What has changed in students' thinking from the previous level?}

\section{What do organisms need to grow and survive?}

0 There is a shift from identifying specific materials from the environment (i.e. food) to identifying specific substances in food (i.e. sugar) for growth and survival. Food is now seen as made of molecules and as providing a source of building material and energy (chemical potential energy).

o Students now recognize the importance of oxygen molecules in reacting with food molecules to provide energy that organisms can use. An understanding of the need for energy to support growth and function emerges here - primarily as the ability to identify energy sources and some key energy transfer events.

o A fundamental distinction is refined and elaborated at this level between plants which can produce their own food, through photosynthesis, and animals and decomposers which must obtain their food by consuming other organisms, dead or alive.

\section{What do organisms do with things they get from their environments?}

o There is a shift in student explanations of how organic matter flows through organisms: before students' explanations suggested that somehow internal organ systems moved food around the body, now it is clearer that organ systems are involved in moving food derivatives around inside plants and animals so that every cell can get the necessary molecules from food in order to build cellular materials or take advantage of the chemical energy in food molecules.

\section{How are organisms connected to their environment?}

o There is a shift in thinking from simple interactions between components of an ecosystem such as the sun, plants, animals, and decomposers to more complex realizations that carbon matter and forms of energy are flowing through the ecosystem from one organism to another, typically beginning with plants.

[Note on general trends in explanations at this level: Although students remain capable of needs-based explanations, they should develop explanations that focus on movement and transformations of matter within and between organisms.] 


\section{Flow of Matter \& Energy in Ecosystems: Level 3}

\section{Student Ideas \& Boundaries}

\section{Prior Ideas, Naive Conceptions, Challenges}

Students do not easily recognize food as being made of atoms and molecules or that the parts of animals or plants are made of atoms and molecules as well. They also do not recognize that these are similar chemical materials, an important step to realizing how the breakdown and synthesis of food substances can generate substances to support growth (C.W. Anderson and colleagues, unpublished data).

Students often think plants get food from the soil as opposed to making it themselves (Bell, 1985).

Students' understanding of energy is just developing. Students can identify energy forms and energy transformations and transfers in simple systems (Nordine, Krajcik, et al., 2010), but they may experience some challenge in applying them to living systems.

Students might not consistently apply principles of conservation of matter and energy to living system. (Mohan et al., 2009; Leach, Driver et al, 1996).

When developing an understanding that matter is composed of atoms and molecules (which would occur at about this time in school), students may confuse the scale size of atoms relative to cells, often confusing atoms for cells (Arnold, 1983). This may pose some challenge in relating how molecules relate to cell structure.

Some students face challenges in relating cells to organ systems in multicellular organisms (Flores, 2003). Some students are unclear as to how molecules are distributed after digestion to other parts of the organism (Mohan et al, 2009; Rowlands, M, 2004; Berthelson, 1999). Moreover some students may not appreciate that digestion is a chemical process and tend to consider it a physical process only (Rowlands, M, 2004).

\section{Useful or Productive Ideas (stepping stones)}

Ideas that allow students to trace the flow of matter and energy are useful here, including a more complete explanation for how matter is broken down inside of organisms and distributed to other parts of the organisms, as well as the ideas that cells are composed of matter and that organism growth is a process that involves successive cell reproduction. (C.W. Anderson, unpublished data).

Students should be able to distinguish between organic matter which has stored chemical energy that living organisms can utilize and other compounds which can not be utilized as a source of chemical potential energy, but they do not need more elaborate analyses of types of organic matter.

[Note, This is a deliberate oversimplification. We recognize that some organisms utilize other sources of chemical potential energy that are inorganic matter, but research on student learning in this area suggests that this oversimplification may still be useful for students when they are expected to think about bonds in compounds breaking and reforming during cellular respiration at the next level (C.W. Anderson, personal communication).]

The four forms of energy implied in the students explanations related to energy under focus questions 2 and 3 above -- light, heat, chemical energy and motion/metabolic functioning-are sufficient at this level (C.W. Anderson, unpublished data). Identifying energy sources may be leverage to developing other ideas about energy later. 


\section{Flow of Matter \& Energy in Ecosystems: Level 3}

Students should be able to explain that organic matter includes both simple and very complex molecules containing $\mathrm{C}, \mathrm{H}, \mathrm{O}$ [it would be good for students to realize also that cells also are composed of similar molecules with these same substances, but that might not develop until high school] (C.W. Anderson, unpublished data).

\section{Boundaries}

Students do not have to understand any detailed molecular structures of food used by living organisms, such as proteins, carbohydrates, or fats, but they should realize they all have some types of atoms in common.

[Note, that while our explanations focus on sugar, it does not imply that students at this level should avoid exploration of proteins or fats as types of food. Certainly some researchers have been able to get students in middle school to think in a rudimentary way about the structure of these different molecules (J. Roseman, AAAS Project 2061, personal communication). However, sugar is the simplest food molecule for students at this level to think about and it a plays a central role in supporting the growth and function of organisms that can produce their own food.]

Students do not have to identify subcellular locations for where processes like photosynthesis and cellular respiration take place. Knowing that they occur inside of the cells of these organisms is good enough. The distinction between prokaryotic and eukaryotic cells is not important at this level.

Students should not be expected to develop an understanding of the details of any specific chemical reaction taking place inside of living organisms until they have decent understanding that atoms and molecules make up matter and can understand that atoms are rearranged during chemical reactions (which might not develop until the end of middle school or early high school.

Enzymes would not be expected in students' explanations at level 3 with regard to processes such as cellular respiration, digestion, and photosynthesis. However, students at this level would be expected to conceive of cellular respiration as a chemical reaction that goes on inside of organisms. There is even some debate as to whether this is needed at the next level, level 4 . What is clear is that at level 4 , students should be able to apply principles such as the conservation of matter and the conservation of energy.

\section{Rationale and Notes}

o The identification of specific substances needed for growth and functioning in animals as well as internal cellular processes for transforming materials into other substances that are used to support growth is essential to tracing matter. Students should be engaged in tasks that focus their attention on the relationship between the weight of materials taken in and changes in organism growth as a function of changes in that organism's weight. While students are likely to have rudimentary understandings of the composition of matter, they should be capable of thinking about specific inputs and relationships between those inputs and changes in organism growth and function.

o It is likely the energy story cannot begin until middle school, because students likely need to develop better models for the structure and transformation of matter first--in particular the idea that atoms rearrange during chemical reactions and the total number of atoms remains the same before and after the reaction (conservation of matter). Such models of matter might not develop until the end of middle school with appropriate instruction. Moreover students are also likely to need experience identifying a variety of energy types and energy transfer or transformation events to non-biological systems before they can apply the ideas to living systems. While the NRC framework does not introduce ideas about energy at middle school for the flow of matter and energy in ecosystems (LS2.B), they are introduced in the context of the flow of matter within organisms (LS1.C). There is some evidence that middle school students, with appropriate instruction, can, however, develop some 


\section{Flow of Matter \& Energy in Ecosystems: Level 3}

rudimentary understandings of energy at middle school and apply them to living systems (Nordine, Krajcik et al, 2010); therefore, it is reasonable to expect students to incorporate initial ideas about energy into their explanations for biological phenomena in middle school.

o In students' explanations for what organism do with things they get from the environment, we elaborated on the process through which food is used in organisms to support growth, in particular the explanation includes details such as food is digested in organ systems and transported throughout the body of the organism so that it can be incorporated into the body structures of the organism. This is key to understanding the flow of matter in living systems and is not properly addressed in most curricula, leaving many students who go on to college without a proper understanding of how food contributes to growth (C.W. Anderson, unpublished data. [We note that these ideas were in earlier drafts of the NRC framework for the flow of matter in organisms and we retained these ideas.]

\section{Notes on how students demonstrate and apply their knowledge (reflected in Learning Performances)}

o In constructing explanations or arguments students will draw upon the idea that food substances are made of molecules and rudimentary models of how matter is transformed as a result of chemical reactions and that these processes occur inside of cells.

o In constructing explanations or arguments, students will draw upon an emerging understanding of energy and identify energy sources and some basic energy transfer events so they can begin to trace energy [Previous explanations did not include energy].

o In constructing explanations, designs, and arguments students should show a beginning ability to trace the flow of matter within and between organisms and between the environment and organisms: this ability is reflected in both students' more sophisticated explanations, justifications, and their plans for how and what organisms do with matter.

o In designing investigations students should be able to increasingly focus on the intake of specific materials and changes in biomass (i.e. students show an increasing sophistication in measuring amounts of living matter (dry mass) and thinking about the continuity of material kinds between food sources and living organisms).

\section{Sample Learning Experiences \& Learning Performances (LE/LP)}

\section{Explaining, predicting, developing explanatory models (cluster 1 )}

LE/LP 1. Construct a model to show how mass is transferred between organisms in an ecosystem that includes plants, animals, and decomposers. Criteria:

o Students should recognize that plants gain mass from carbon dioxide in the air rather than exclusively from water and soil. Example justification: The total mass of a plant and the soil it is growing in increases as the plant grows.

o Students should recognize that both animals and decomposers lose mass from the food they ingest to carbon dioxide as they function (i.e. moves or exercises). Example justification: the total mass of a growing animal and its food declines as an animal exercises.

LE/LP 2. Construct an explanation for how animals and plants get the energy they need to perform functions like growth, repair of body parts, and movement (perhaps intracellular movement for plants).

Criteria:

o Students' explanations should include energy sources for animals and plants and account for when and where it is transferred.

o For animals, students should identify the chemical energy found in food as an energy source that can be transformed or transferred 


\section{Flow of Matter \& Energy in Ecosystems: Level 3}

to carry out functions like movement, or carry out work like building body parts during growth or repair

o For plants, students should identify sunlight as the source of energy that can be converted to chemical energy that can then be transformed or transferred to carry out functions such as moving things inside of plant cells or building plant parts during growth [this is a new performance that previously could not be done at this point since ideas about energy emerge in students' explanations or models; they primarily can identify energy sources and identify some energy transformation or transferal events].

\section{Designing investigations, collecting, representing, and analyzing data (cluster 2)}

LE/LP 3. Design an investigation and collect and represent data that allows one to determine what factors contribute to growth in plants (as measured by an increase in biomass).

Criteria:

o Students' designs should account for critical factors such as access to light, carbon dioxide, and water. Students will have to account for irrelevant or negligible factors such as weight of the container, water and soil.

LE/LP 4. Design an investigation and collect and represent data that allows one to determine what factors contribute to the growth of simple organisms (e.g. worms, frogs, or fish).

Criteria:

o Students' designs must account for critical factors such as access to food. Students will have to account for irrelevant or negligible factors such as weight of the container, and weight of water.

LE/LP 5. Analyze a data set that includes measures of carbon dioxide production or absorption under specific conditions by plants, animals, and decomposers. Conditions should examine carbon dioxide production during periods of active growth (for all organisms) or obvious movement (e.g. exercising animals).

Criteria:

o Students' analysis should identify patterns where carbon dioxide production is detected for all organisms and that it changes especially during periods of active growth or movement. Students must also recognize that plants are the only organisms among the set examined that absorb detectable measures of carbon dioxide.

\section{Constructing scientific argumentations (cluster 3)}

\section{LE/LP 6. Construct an argument that plants need food.}

Criteria:

o Students' argument should include the idea that food provides building materials and energy for living things. Plants grow and as a consequence their mass increases; therefore, plants must obtain matter which has mass. This mass comes from food made by the plants from the materials they take in, carbon dioxide and water. Growth also requires energy. The argument should draw on the idea that energy can be transferred from one system to another or converted from one form to another, but is conserved. Plants must get this energy from some source, namely food compounds that they have produced by photosynthesis.

LE/LP 7. Construct an argument that in complex multi-cellular organisms, such as mammals, energy is released from food in the body's cells. Criteria: 


\section{Flow of Matter \& Energy in Ecosystems: Level 3}

o Students' arguments should include a chain of reasoning that establishes that a chemical reaction is occurring inside cells. This includes establishing that food is broken down into molecules; that these molecules and oxygen are carried around to each and every cell in the body and; the cells are releasing different materials (carbon dioxide and water) as well as releasing energy to do biological work (growth, cell division, movement, etc.).

LE/LP 8. Critique explanations that account for a data set that relates changes in biomass and carbon dioxide concentrations in plants and animals. Data can focus on changes in carbon dioxide levels in the immediate vicinity of the organism.

Criteria:

o Students' critiques should consider the different and similar processes by which these different organisms take in and transform matter.

o Students should recognize that plants, unlike animals, take in carbon dioxide and can transform it into a form that can be incorporated into the structures of the plant (via photosynthesis and biosynthesis).

o Students should then explain that when more carbon dioxide is available more biomass can be generated in plants.

o Students should also recognize that animals do not gain any mass regardless of carbon dioxide level because they do not engage in photosynthesis.

Learning performance focused on matter were informed by work of Mohan et al., 2009. Learning performances focused on energy were informed by curriculum materials developed by B. Reiser and colleagues for middle school students (http://www.umich.edu/ hiceweb/iqwst/index.html). 


\title{
Flow of Matter \& Energy in Ecosystems: Level 4
}

\begin{abstract}
LEVEL 4 (Grades 9-12): Atomic-based explanations
Overview: Students explanations and learning performances have the following characteristics:

Living things require specific elements (namely carbon) found in specific compounds to support growth and function. Students have a better ability to trace the flow of matter between organisms because there is even greater ability to understand the chemical and cellular processes involved in the transformation and movement of organic and inorganic matter within and between organisms that can be applied to all organisms in an ecosystem, including plants, animals, and decomposers. Principles of conservation of matter and conservation of energy also are applied to further allow students to trace the flow of matter and energy. In general, students at this level apply rules and principles to understand and explain natural phenomena in living systems.
\end{abstract}

\section{Level 4 Student Explanation or Explanatory Model}

\section{Focus Question 1: What do organisms need to grow and survive?}

As a general rule, matter cycles and energy flows through different levels of organization of living systems, and between living systems and the physical environment, matter and energy are conserved in each change.

All organisms are made of the same basic materials, including water, and particular biological molecules such as carbohydrates, lipids, nucleic acids (i.e. DNA) and proteins (all have carbon as their basic building block). As a result all organisms need to get the same substances to support their growth. These substances can be found in food. Animals and decomposers consume these materials in the food they eat. Plants are unique in their ability to capture energy from sunlight and use it to construct these materials from inorganic ingredients: carbon dioxide, water, and soil minerals.

\section{Focus Question 2: what do organisms do with things they get from their environments?}

As a general rule, the growth and functioning of all organisms occurs though chemical processes in which atoms are rearranged into new molecules inside cells. These chemical reactions involve the transformation of matter (in which atoms rearrange to form different substances). These chemical reactions also involved the transfer of energy (from one form of energy to another, or from one system of interacting molecules to another). Both matter and energy are conserved during transfer events within organisms.

Matter: Plants utilize compounds from sugar generated through photosynthesis along with soil minerals to make amino acids and other carboncontaining molecules. These molecules can then be assembled into larger molecules like complex carbohydrates, lipids, nucleic acids, and proteins (biosynthesis) that can either be incorporated into plant structures or perform biological activity (e.g. enzymes) inside of plants. Animals and decomposers grow by digesting carbohydrates, lipids, nucleic acids and proteins (i.e., breaking them down into smaller organic molecules) and assembling them into new larger biological molecules (biosynthesis) that can then be incorporated into animal structures or perform biological activity inside of animals. Therefore, all organisms engage in biosynthetic chemical reactions to support growth and functioning.

Energy: Through photosynthesis, plants take energy from light to form sugar molecules (food) containing carbon, hydrogen, and oxygen. Photosynthesis and the production of sugars occur in specialized sub-cellular locations/structures [chloroplasts] that are only found in plants [and other photosynthetic micro-organisms]. During this process water and carbon dioxide react to form sugar and oxygen. 


\section{Flow of Matter \& Energy in Ecosystems: Level 4}

Through cellular respiration, which takes place in most organisms, including animals, plants, and most decomposers, a form of energy is generated that drives the chemical reactions that support growth and functioning. In this cellular process, oxygen reacts with food (i.e., carbohydrates, lipids, nucleic acids and proteins) that form carbon dioxide and water. During this cellular process energy is transferred from one set of molecules to another set of molecules [e.g. ATP] as bonds are broken and reformed during chemical reactions that take place in special subcellular locations or structures inside of the cells in plants, animals or decomposers [e.g. mitochondria]. This reaction, like the burning of wood or coal, involves oxygen, is highly controlled and energy is transferred to other molecules that can be transported to cells all over the organism to drive other chemical reactions. These chemical reactions support cellular processes such as biosynthesis to support growth or reproduction or movement of molecules within and across cells to support cellular functioning. Biological work can include not only movement but the synthesis of large macromolecules such as protein or DNA needed during growth. Since principles of energy conservation apply to living systems, inputs of energy are always equal to energy that is stored, used, released (as heat), or excreted from organisms. Thus any change in input must be accounted for by changes in energy stored, used, released, or excreted-or vice versa.

[Note, we recognize some organisms get the energy they need from inorganic compounds, such as hydrogen sulfide - which is the case for organisms found in deep ocean vents - but most ecosystems examined in high school are likely to not include these organisms so we did not specifically include such cases in the explanation. We do feel that if students understand the general principle embedded in the explanation above -- "compounds consumed by living organisms are transformed during which energy is transferred from one system of molecules to another as bonds are broken and reformed" -- that students should be able to make sense of these other phenomena as well if they are introduced to them.]

\section{Focus Question 3: How are organisms connected to each other and their environments?}

In general: All processes that occur at the cellular, organismal, and population scales are part of the general processes of energy flow and carbon cycling in ecosystems and principles of conservation of matter and energy apply throughout every part of the ecosystem.

Matter Flow (Carbon Cycling): Carbon (and other key elements used by biological organisms such as phosphorus and nitrogen) is recycled within the ecosystem. The chemical elements that make up the molecules of organisms pass through food webs and are combined and recombined in different ways. Carbon is transformed from carbon dioxide and water into the carbohydrates, lipids, nucleic acids and proteins that make up living organisms through processes that occur in organisms such as photosynthesis, digestion, and biosynthesis, and is subsequently returned (recycled) back to the atmosphere as carbon dioxide through cellular respiration which occurs in all organisms. Only a small fraction of the matter consumed at the lower level of a food web is transferred upward.

Energy Flow: Energy flows through the ecosystem moving from one place to another. Some of it is used at each step to support life activities, and typically at each transfer some is released as heat into the environment- it is not recycled like matter (however energy conservation principles still apply). Energy flow begins with plants where it is transferred through cellular processes such as photosynthesis, cellular respiration, biosynthesis, and digestion to support growth and other life functions. Some energy then flows to animals where it is transferred through cellular processes such as digestion, cellular respiration, and biosynthesis to support growth and function. Organic matter found in dead organisms or waste products from living organisms can flow to decomposers where similar processes of digestion, cellular respiration, and biosynthesis can occur. Since, only photosynthetic organisms like plants or some algae can produce their own food using energy from the sun, and other organisms eat those plants or consume other animals that ate the plants, all matter in most ecosystems can be traced back to plants. Every energy transfer results in some energy released as heat into the environment that cannot be used to do work, eventually most of the energy that goes into an entire ecosystem is released as heat into the environment as organisms in the ecosystem utilize energy in food to grow and function. Hence, as one moves up different levels in the ecosystem less and less energy is available to organisms. 


\section{Flow of Matter \& Energy in Ecosystems: Level 4}

Because matter transformations and energy transferal events occur within and between organisms and between the environment and organisms, all organisms are intimately connected to the other organisms and the physical surroundings (e.g. atmosphere, geosphere, etc.) in their environment for their matter and energy needs.

[Note, again we recognize that some food webs begin with non-photosynthetic organisms, such as bacteria that use inorganic compounds as a source of energy, but we maintain students should understand the ecosystem they are most familiar with first and apply those principle to these less common ecosystem.]

\section{What has changed in students' thinking from the previous level?}

\section{What do living things need to grow and survive?}

o There is a shift here in realizing that plants, animals, and decomposers share important similarities: they all use the same organic molecules and utilize the same cellular processes to support growth and survival. However, plants have the unique ability to produce their own food through photosynthesis.

o There is also a continued refinement in understanding of the specific materials that all organisms need to grow and survive, in particular carbohydrates, nucleic acids, and lipids which can be obtained in food.

\section{What do living things do with things they get from their environment?}

o Students' explanations now recognize that energy and matter are flowing through multiple biological levels-e.g. moving from the ecosystem level, to the organismal and the cellular and atomic-molecular levels. Students' explanations more fully recognize that chemical reactions allow for the transfer of energy and transformation of matter within organisms and ecosystems.

o Students' explanations now include a more refined and complete explanation for how energy is utilized from food as well as how energy is transferred to other systems of molecules during cellular respiration. Students also apply principles of energy conservation to explain the stock and flow of energy in biological organisms.

o Students' explanations now show evidence that they can trace energy and matter down to sub-cellular locations inside of organisms. In other words, students' explanations recognize that cellular respiration is carried out in specialize sub-cellular locations (i.e. mitochondria) and that biosynthesis processes occur elsewhere in the cell-- in particular in photosynthetic organisms the generation of sugars (food) occurs in the chloroplasts.

o Students now recognize that plants, outside of photosynthesis, engage in all the same basic process as animals and decomposers, namely chemical digestion, biosynthesis, and cellular respiration.

o Students' explanations now include broader instances of biological work and include biosynthesis of macromolecules during growth in addition to movement.

o Students' explanations now have a more complete account for how energy and matter are related. In other words, students' recognize that energy is stored in the bonds of molecules and can be transferred to do work such as synthesizing materials for growth. Thus students now realize that matter and energy are flowing together as matter transforms. Students also realize that while total energy is conserved if you keep track of all the heat, the usable energy isn't the same at the end (i.e. energy is not recycled). 


\section{Flow of Matter \& Energy in Ecosystems: Level 4}

\section{How are organisms connected to their environment?}

o At this level the matter and energy can be represented and recognized as flowing in specific ways [carbon matter is recycled and energy is flowing through and out -- much energy being lost as heat]. This represents the use of models of energy and matter to account for the behavior of matter and energy in ecosystems instead of a focus on individual actors that was characteristic of previous levels.

o There is also a better picture of how carbon cycles between organisms and the environment in that they realize carbon is the central element which is shuttled between organisms and the environment through various chemical transformations that occur within organisms.

o Principles of matter and energy conservation are applied at every point of interaction in the ecosystem.

\section{Student Ideas \& Boundaries}

\section{Prior Ideas, Naive Conceptions, Challenges}

A well-developed model of matter in which students understand matter to be composed of atoms and molecules and that those atoms rearrange during chemical reactions and principles of conservation of matter apply is not always easily developed (Smith et al., 2006). However if students are given time to develop these ideas as outlined in the progression for matter, they may be more likely to develop these understandings by the end of middle school (Krajcik, McNeill, et al., 2008).

The basic understanding that most High School students never achieve is that compounds like glucose and ATP store energy in chemical bonds, not in the atoms themselves, so that oxidation of organic molecules releases energy without destroying atoms. A couple of other key difficulties include: 1) distinguishing between the "breaking down" of food molecules that occurs in digestion and the "breaking down" that occurs in cellular respiration, and 2) recognizing biosynthesis and cellular respiration as alternate pathways for digested food. (C.W. Anderson and colleagues unpublished interviews).

\section{Boundaries}

It is unnecessary to get into the details of the biochemical pathways for cellular respiration, photosynthesis, or biosynthesis. It is sufficient for students to understand that atoms are rearranged during these processes and that energy is transferred from one system of molecules to another system of molecules. Simply understanding that the arrangement of the atoms in these molecules is changed to generate different molecules is sufficient. Similarly it is unnecessary to get into the details of ATP; it is sufficient for students to realize a new set of molecules are formed that can be transported all around the cell and to other cells all over the organism where the energy in ATP can be transferred to yet another set of molecules.

It is also unnecessary to understand the detailed molecular structure of biological compounds - however it is important for students to realize that these molecules have carbon, hydrogen, and oxygen as key and common elements.

Although mitochondria and chloroplasts were alluded to as part of the process of cellular respiration and photosynthesis in students' explanations, it would be unnecessary to go into the details how these organelles are structured or any of the detailed molecular events that go on inside them.

\section{Rationale and Notes}

o Students are developing more refined explanations for the flow of matter and energy in ecosystems as their conceptual models for matter and energy improve as well their models for how organisms are structured (which includes describing the relationships between whole organism, 


\section{Flow of Matter \& Energy in Ecosystems: Level 4}

tissue, cellular, and atomic-molecular levels). They also are developing more refined understandings of the inter-dependent connections between organisms in an ecosystem as well as matter in the environment and organisms in the ecosystem. The additions of specific processes, materials, and locations for processes in students' explanations reflect this more refined level of explanation.

- We added ideas about subcellular locations of processes like cellular respiration and photosynthesis because they can provide evidence that these processes occur at a subcellular level and that animals and plants carry out similar functions (i.e. both have mitochondria) whereas plants are special and have chloroplasts to carry out photosynthesis [These ideas were in earlier drafts of the NRC framework for the flow of matter in organisms].

o We selected several learning performances that rely on virtual data at this level rather than data from wet labs because they show, perhaps more clearly, how students can demonstrate and apply their increasingly more sophisticated explanations and models that include atomic-based explanations. If students hold such explanations, they should be able to make some sense of radio-labeled carbon tracing experiments [of course with some rudimentary explanation for how the label works]. Similarly data sets that could come from calorimetry experiments focusing on energy inputs and outputs could be interpretable by students at this level in the progression energy, and the inter-dependent connections between organisms and the environment. Students should be able to analyze, interpret, and explain more complex natural phenomena that involve the flow of matter and energy in ecosystems such as changes in ecosystems when one factor in the ecosystem is perturbed. It could be argued explaining such phenomena is perhaps one of the main reasons why students should understand the flow of matter and energy in ecosystems today, thus, we also included a learning performance with this type of phenomenon.

\section{Notes on how students demonstrate and apply their knowledge (reflected in Learning Performances)}

o In constructing explanations for growth and function students should be able to draw upon more refined understandings of matter and matter transformation by using atomic-molecular model of matter and include more refined understandings of how energy is utilized from food. Principles of conservation of energy and matter are also applied here.

o In constructing explanations, designs, and arguments students should have a more refined ability to trace matter and energy between organisms and between the environment and organisms: in particular the ability to trace matter in explanations and investigations depends on their ability to recognize carbon as the key element that flows between organisms and between the environment and organisms.

o In analyzing data students are capable of interpreting and explaining more complex data sets reflecting the flow of matter and energy in organisms and ecosystems [previously, notions of energy flow in living systems as just developing].

o In constructing explanations and arguments students should also have a more refined account for how matter and energy flows through organisms and ecosystems as they develop better models for how matter is cycled and energy is transferred.

\section{Sample Learning Experiences \& Learning Performances (LE/LP)}

LE/LP 1. Construct an explanation for how matter and energy is obtained that enables animals, plants, and decomposers to grow or survive. Criteria:

- For matter, the explanations should focus particularly on the carbon flow into organisms (in carbon dioxide in plants and food in other organisms) and out of organisms (in carbon dioxide) and within the sequence of common biochemical processes (digestion/catabolism and cellular respiration and biosynthesis) [this is a shift from the previous level that did not emphasize carbon and common processes as much]. 


\section{Flow of Matter \& Energy in Ecosystems: Level 4}

o Should also note that matter can be used as both cellular building blocks if shuttled through biosynthesis pathways or a source of energy if shuttled through cellular respiration.

o For energy, not only should students be able to identify energy sources (e.g. food for animals and plants), but important transfer events such as sun to chemical potential (in food produced in plants) and chemical potential to thermal as chemical reactions take place. Student's explanations should indicate that during chemical reactions, which underlie key processes (photosynthesis, cellular respiration, and biosynthesis) that energy is transferred from one system of molecules to another.

LE/LP 2. Construct explanations that account for a data set_that tracks flow of radio-labeled carbon in a mini-ecosystem.

Criteria:

o Students' explanations should consider photosynthesis and biosynthesis in plants as well as digestion, biosynthesis and cellular respiration in animals and decomposers.

o Students' explanations should also consider conservation of matter as matter undergoes various chemical transformations.

\section{Designing investigations, collecting, representing, and analyzing data (Cluster 2)}

LE/LP 3. Analyze a data set provided to them that reflects the flow of radio-labeled carbon matter to determine where carbon matter ends up in organisms constituting a mini-ecosystem (e.g. could show data that track radio-labeled glucose fed to animals and represent its accumulation in the body of animals, as well the incorporation of the labeled carbon into structures of plants and decomposers in a closed and controlled mini-ecosystem).

Criteria:

o Students' analysis should identify patterns where radio-labeled carbon ends up in the body structures of animals as a result of digestion and biosynthesis - or body structures of plants as a result of photosynthesis and biosynthesis.

LE/LP 4. Students analyze, identify, and interpret patterns in a data set provided to them that includes measures of energy for energy inputs, energy used to do work, energy lost as heat or excreted as waste for an animal during different states (e.g. rest, actively moving, actively growing, with less food, etc.), and energy retained and excreted.

[Note: Data from a variety of sources could be provided for each these quantities (e.g. energy taken in could be measured by bomb calorimetry of food

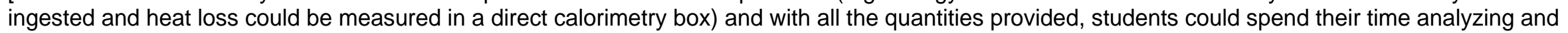
interpreting the data set]. Criteria:

o Students capable of applying conservation of energy and matter should identify a number of patterns. In particular, the total amount of energy retained and secreted should equal the amount of energy ingested (in food), minus the amount of energy used to do work on the environment (e.g. a mouse of certain mass walking a certain distance), minus any energy in waste minus any heat produced.

o Students should realize any number of interrelationships among the above variables that are less than the whole of energy balance: more energy intake is directly related to body mass, all else being the same; more work is indirectly related to body mass, all else being the same. 


\section{Flow of Matter \& Energy in Ecosystems: Level 4}

o Students should also be able to describe why it makes sense that these data change the way they do in the data set during different physiological states.

\section{Constructing scientific arguments (cluster 3 )}

LE/LP 5. Critique explanations_for perturbations in real world ecosystems where students use models of matter transformation and the connections between organisms and their environment to account for the consequences [e.g. Lake Victoria in Africa where overgrowth of algae created an increase in dead and decomposing matter and subsequent oxygen depletion at lower lake depths and consequently "dead-zones" in the lake where no plants or animals could live].

Criteria:

o Students should apply models of how food is utilized through chemical processes inside of cells such as photosynthesis and cellular respiration to account for changes in available food, or key environmental compounds like oxygen, carbon dioxide, or essential minerals to support growth of organisms. They must also apply this reasoning to whole populations of a species and rely on their knowledge of how plants, animals, decomposers, and components in the atmosphere and geosphere are connected in order to properly account for and critique other explanations. Students must also realize that matter is recycled, while energy is not.

LE/LP 6. Critique explanations for observations of trophic levels in food webs (e.g. why is there only a limited number of trophic levels in a food web, or why are there fewer organisms at higher trophic levels in a food web).

Criteria:

o Students should apply models of how food is utilized through chemical processes inside of cells like photosynthesis and cellular respiration to account for changes in available food, or key environmental compounds like oxygen, carbon dioxide, or essential minerals to support growth of organisms.

o They should also realize that food at upper levels in the food web is only available through the food that animals eat, that energy flows in-and eventually out-of the system, and that many activities that organisms need to perform (e.g. growth and function) rely on a source of energy- which is food. Part of food goes to building body structures, much goes to driving chemical reactions like cellular respiration, biosynthesis, and maintaining normal cell function.

o They should point out that transfer of energy from one level to another is not $100 \%$ efficient; some is lost at each step. Therefore, less energy and less matter are available to support life at higher levels. 


\section{References}

Bell, B. (1985). Students' understanding of plant nutrition. In B. Bell, M. Watts, \& K. Ellington (Eds.), Learning, doing, and understanding in science. The proceedings of a conference, 11-13 July 1984 (pp. 36-39). London: SSCR.

Berthelson, B. (1999). Students Naive Conceptions in Life Science. Michigan Science Teaches Association Journal. Spring, 13-19. http://www.msta-mich.org

Flores, F., Tovar, M., \& Gallegos, L. (2003). Representation of the cell and its process in high school students: An integrated view. International Journal of Science Education 25(2), 269-286.

Inagaki, K., \& Hatano, G. (2002). Young children's naive thinking about the biological world. New York: Psychology Press.

Krajcik, J., K. L. McNeill, et al. (2008). "Learning-goals-driven design model: Developing curriculum materials that align with national standards and incorporate project-based pedagogy." Science Education 92(1): 1-32.

Leach, J., Driver, R., Scott, P., \& Wood-Robinson, C. (1992). Progression on conceptual understanding of ecological concepts by pupils aged 5-16. Leeds, England: Center for Studies in Science and Mathematics Education, University of Leeds

Lehrer, R., \& Schauble, L. (in press). Seeding evolutionary thinking by modeling its foundations. Science Education.

Lehrer, R., \& Schauble, L. (2000). Model-based reasoning in mathematics and science. In R. Glaser, Advances in instructional psychology, Vol. 5 (pp. 101-159). Mahwah, NJ: Lawrence Erlbaum Associates

Lehrer, R., \& Schauble, L. (2004). Modeling natural variation through distribution. American Educational Research Journal 41(3), 635-679.

Lehrer, R., Schauble, L., Carpenter, S., \& Penner, D. (2000). Designing classrooms that support inquiry. In J. Minstrell \& E. V. Zee (Eds.), Inquiring into inquiry learning and teaching in science (pp. 80-99). Washington, DC: American Association for the Advancement of Science. 
Lehrer, R., Schauble, L., Carpenter, S., \& Penner, D. E. (2000). The inter-related development of inscriptions and conceptual understanding. In P. Cobb, E. Yackel, \& K. McClain (Eds.), Symbolizing and communicating in mathematics classrooms: Perspectives on discourse, tools, and instructional design (pp. 325-360). Mahwah, NJ: Lawrence Erlbaum Associates.

Metz, K. E. (2011). Disentangling robust developmental constraints from the instructionally mutable: Young children's epistemic reasoning about a study of their own design. Journal of the Learning Sciences, 20, 50-110.

Mohan, L., J. Chen, et al. (2009). "Developing a multi-year learning progression for carbon cycling in socio-ecological systems." Journal of Research in Science Teaching 46(6): 675-698.

Nordine, J., Krajcik, J. \& Fortus, D. (2011). Transforming Energy Instruction in Middle School to Support Integrated Understanding and Future Learning. Science Education 95 (4):670-699.

Rowlands, M. (2004). What do children think happens to the food they eat. Journal of Biological Education. 32(4)167-171

Smith, C. L., M. Wiser, et al. (2006). "Implications of Research on Children's Learning for Standards and Assessment: A Proposed Learning Progression for Matter and the Atomic-Molecular Theory." Measurement: Interdisciplinary Research and Perspectives 4(1-2): 1-98.

Wiser, M. and C. L. Smith (2008). Learning and Teaching about Matter in Grades K-8: When Should the Atomic-Molecular Theory Be Introduced? International Hanbook of Research on Conceptual Change. S. Vosniadou. New York, Routledge 


\section{Appendix A: \\ Consultants Working on Hypothetical Learning Progressions}

\section{Structure, Properties, and Transformations of Matter}

- Joseph Krajcik, Director of the Institute for Research on Mathematics and Science Education, Michigan State University; Chair of Working Group

- Jacob Foster, Director of Science Technology and Engineering, Massachusetts Department of Education

- Fred Goldberg, Professor of Physics, Center for Research in Mathematics \& Science Education, San Diego State University

- Shawn Stevens, Research Investigator, School of Education, University of Michigan

- Marianne Wiser, Associate Professor \& Chair, Hiatt School of Psychology, Clark University

\section{Flow of Matter and Energy in Ecosystems}

- Brian Reiser, Professor of Learning Sciences, School of Education \& Social Policy, Northwestern University; Chair of Working Group

- Charles (Andy) Anderson, Professor, Department of Teacher Education, Michigan State University

- Jennifer Hicks, Science Curriculum Specialist, Indiana Department of Education

- David Kanter, Director of SciPlay, New York Hall of Science

- Richard Lehrer, Professor of Science Education, College of Education \& Human Development, Vanderbilt University 


\section{Appendix B: \\ List of science practices and design principles to use in developing hypothetical learning performances}

Learning performances were not identified and developed arbitrarily for the hypothetical learning progressions. We developed a design scheme that deliberately considered a set of science practices to match with content ideas (i.e. students' explanations of models). We have developed a list of seven practices that were then grouped into three clusters so that the practices could be systematically represented at each level in the progression. The set of science practices considered in the development of learning performances and the design principles using these practices and clusters are articulated below.

Since learning performances also reflect insight from current research on science learning and how students can achieve science proficiency, the end of this appendix describes how the hypothetical learning progression and learning performances relate to the "four strands of science literacy" (i.e. ways in which students learn and become proficient in science (Duschl, Schwiengruber, \& Shouse, 2007).

\section{Selecting science practices to include in learning performances and matching them with students' explanations or models}

These are the seven practices that the team of consultants working on the progression came to agreement on. The practices are consistent with the Conceptual Framework for New Science Education Standards from the National Research Council (NRC, 2011), the College Board Standards for College Success (CBSCS, 2009), and current research on scientific practices such at the NRC report Taking Science to School (Duschl et al., 2007).

There is a need to be able to easily see for each learning performance which content it uses and which practice or practices form the work of the performance. One way to make this more explicit is to use consistent language in the learning performances that makes it easy to determine which practice is being used in a performance. Thus, following each practice below are some of the terms that we use in the corresponding learning performances, in combination with particular aspects of scientific content (i.e. students' explanations or models).

- Questioning: Asking and refining scientific questions that can be investigated empirically (CBSCS \#1, NRC).

- Finding, evaluating, and communicating information: Finding, interpreting, evaluating, and communicating scientific information (NRC, Common Core ELA).

- Designing Investigations: Designing investigations, including hypothesizing, generating and selecting attributes and measures of these attributes, and data collection plans (CBSCS \#2, NRC).

Example use in performances:

o Design an experiment to test whether... [question]

- Design an experiment to show that... [claim]

- Collecting, representing, and analyzing data: Collecting, representing, analyzing and interpreting data; including searching for regularities and patterns in observations and measurements (CBSCS \#3, NRC). 
- Explanation and prediction: Constructing predictions and explanatory accounts of phenomena, including evidence-based explanations (CBSCS \#4, NRC).

Example use in performances:

- Explain how and why this phenomenon occurs...

- Given a particular set of data, write an evidence-based explanation that explains why the phenomena occurred...

- Predict what will happen to variable $X$ if variable $Y$ changes...

- Modeling: Developing, evaluating, and revising scientific models to explain and predict (CBSCS \#4, NRC).

Example use in performances:

a. $\quad$ Construct a model that explains...

b. $\quad$ Revise a model of...to account for new evidence that...

- Argumentation: Engaging in argumentation to defend or critique scientific questions, designs of investigations, representations and analyses of data, evidence-based explanations, predictions, and scientific models (NRC, missing from CBSCS).

Example use in performances

- Critique how a given data representation reflects the given data...

- Revise a written argument to take account of this particular counter argument...

- Defend a model that accounts for a set of particular phenomena

\section{Clusters of practices}

The motivation for forming clusters of practices is to select particular performances in a principled way to write at each level in the hypothetical learning progression. We do not want to argue that we always have to represent each learning goal with each of the seven practices. That would be a rather overwhelming task. On the other hand, we felt that the sampling of the performances should be systematic, to best reveal what is changing at each grade level and reflect the key practices of science known to support science learning.

The clustering strategy we came to consensus on is not the only way to cluster the practices, but we felt this grouping strategy reflects a naturally overlap of the science practices. For example, explanations can be created by applying a scientific model (such as the particle model) to a phenomenon (such as melting), thus practices 5 and 6 (from the above list) appear to cluster naturally together. Similarly there is a longer list of practices that relate to the process of engaging in empirical investigations such as designing investigations, collecting and representing data, and analyzing data (practices 1-4 from the above list) that cluster together.

The clusters can guide creation of learning performances by making sure we have at least one learning performance from each cluster at each grade band. A learning performance may draw on a single practice from the cluster (such as explanation), or may combine practices (such as argumentation for a model).

The proposal for clusters of practices is as follows: 
Cluster 1 - Explaining, predicting, and developing explanatory models (Practices 5, 6). [Taking Science to School Strand 1: Knowing, using, and interpreting scientific explanations of natural world, Duschl et al., 2007]

Cluster 1 includes practices 5 and 6 from above. This is most directly connected to strand 1 from the NRC report Taking Science to School (Duschl et al., 2007), applying explanations to the natural world. It includes the practices of explanation (practice 5), providing causal, mechanistic, and principled accounts of phenomena in the natural world, and includes evidence-based explanations of scientific phenomena. It also includes the modeling practice (practice 6), in which learners develop and refine explanatory models.

These practices can also be used in performances that would involve strand 4, participation in scientific practices in a community. It depends on how the performances are implemented as assessments. For example, a learning performance in this cluster may be to develop a general model for the effects of competition in ecosystems. The development of the model could involve collaboration and discourse to propose, evaluate, and refine components of the model, making it reflective of strand 4.

Cluster 2 - Designing investigations, collecting, representing, and analyzing data (Practices 1-4). [Taking Science to School Strand 2: Generating and evaluating scientific evidence]

This includes practices 1-4 above. These four practices correspond most directly with strand 2 from Taking Science to School, generating scientific knowledge (Duschl et al., 2007) but if implemented through classroom interaction and discourse could also involve strand 4.

To select investigation performances, the emphasis should be on investigations that are closely tied to the learning trajectories in explaining and predicting practices above. We should describe investigations that could either (a) drive changes in student explanations and predictions by generating evidence in favor of more advanced models or (b) enable students to support their explanations and predictions with arguments from empirical evidence.

Cluster 3 - Constructing scientific arguments (combines argumentation related tasks (practice 7) with practices from either cluster 1 or cluster 2 explicitly). [Taking Science to School Strand 4: Participating productively in scientific practices and discourse]

Students can construct a scientific argument in support of various types of products, ranging from representations of data to designs of investigations, explanatory models, and evidence-based explanations of phenomena. Thus, the third cluster combines the practice of argumentation (i.e. task such as critiquing, defending, or revising an argument) with practices from the investigation cluster or the explanation cluster. To differentiate a learning performance from cluster 1 or cluster 2 from cluster 3 , we include for example, verbs such as "critique" or "defend" a model to indicate it is an argumentation learning performance versus from simply providing or constructing a model which would be categorized as a cluster 1 learning performance. 
Learning performances should be constructed that differentiate investigations, explanations, or models characteristic of the current grade band from prior grade bands. That is, at each level, students would have the task of mounting an argument for the superiority of explanations at their level to explanations characteristic of the grade band below.

\section{Design principles for creating learning performances}

The following design principles should be considered when developing learning performances.

- Clarity: The learning performance should make clear (a) what product is being created (e.g., an experiment design, a data representation, a model, a critique of a conclusion from an experiment), (b) what practice is being used, and (c) what content is being targeted. These should be evident in a concise statement of the performance, e.g., "Students critique explanations for why a liquid expands upon heating." There should also be criteria that follow the learning performance statement which should include content from the student explanation column, and clarify what the particular characteristics the product needs to include. The criteria help to clarify what to look for in a student's performance that would be evidence they have achieved the level of understanding articulated in the progression.

- Show contrast with prior level: Select the performances to make as transparent as possible how the students' practices and explanations are more sophisticated than the prior level. For example, select an investigation performance to find data to uncover problems with prior models, or have students develop a model that accounts for findings that cannot be explained at the prior level. The content in the performance should reflect the key transitions in thinking between levels in the progression (e.g. for the particle model, students at level 3 are not expected to consider inter-particle attractions but at level 4 they are expected to, so phenomena or criteria for evaluating performance must attend to this shift in thinking).

- Breadth: To represent the breadth of scientific practices, include a learning performance from each of the three clusters of practices listed above at each grade level.

- Explicitness in practices: We can have some performances with multiple practices and some that only have one practice explicitly stated. We cannot assume that just because a particular practice is implicit in the performance that it will be part of the expected learning performance unless a verb is attached that makes it a clear expectation. This is especially important for learning performances that fall under the argumentation cluster.

- Appropriateness of performances: The performance must not require a more sophisticated explanation or model in order for students to perform adequately on the task.

- Direct connections between conceptual development and practices: We can write performances that best show how they are connected to column 1 (student's explanation or explanatory model). The practices in the set of learning performances should support the conceptual development story. We are not including practices that are independent of the conceptual development story. Someone reading the learning performance should be able to see how the 
criteria for evaluating the performances reflects the section in the table with students' explanation or model.

- Draw upon evidence: Wherever possible note which performances are grounded in empirical evidence. In other words, if a performance has been shown to support students' conceptual development or has been used with students at a particular age level and they can in fact engage in that performance adequately, it should be noted and included among the set of learning performances. [There eventually will be list of citations that correspond to the set or learning performances] $]^{3}$.

\section{Connection of learning performance in hypothetical learning progression to the strands of science literacy from Taking Science to School (Duschl et al, 2007)}

The connections of the four strands of science literacy to the hypothetical learning progression are summarized in the table below. These are the same strands mentioned in the report Ready, Set, Science (Michaels, Schwiengruber, \& Shouse, 2007). As noted above, strand 1 (essentially using explanations) and strand 2 (essentially investigation practices) are the most explicit in the hypothesized learning progression. Strand 4 (participating in practices and discourse) is potentially reflected in the use of practices in the learning progression, depending on whether these are treated as individual assessments, or as involving learners in a community of scientific practice, as strand 4 is meant to reflect. Strand 3 (understanding the nature and development of scientific knowledge), in contrast, is not reflected independently in the learning performancesbecause this strand is especially important in guiding the engagement of learners in practices and using scientific knowledge. A decision was made not to target the nature of science knowledge as an independent learning goal since it is integral to all the other practices and is always present at some form.

\footnotetext{
${ }^{3}$ Ideally, we should include learning performance for which assessments or instructional tasks have been proven to be effective and reliable at identifying students at a particular level in the progression, or that have informed tasks proven to effectively promote shifts in thinking, but there are few such learning performance available. We have chosen to represent a set of learning performance, that although informed by actual assessment or curriculum work, have yet to be proven directly for their effectiveness in this regard. The learning performances do reflect some key aspects of learning progression perspective however - namely that change in understanding should be examined in the context of how students apply their ideas in more sophisticated ways and learning performance should attempt to identify and place students somewhere along the progression and give some indication of what they know and can do relative to starting and end points.
} 


\begin{tabular}{|c|c|c|}
\hline $\begin{array}{l}\text { Strands from Taking } \\
\text { Science to School }\end{array}$ & $\begin{array}{l}\text { Where to look in the } \\
\text { hypothetical learning } \\
\text { progression }\end{array}$ & $\begin{array}{l}\text { How the hypothetical learning progression is } \\
\text { designed to reflect the commitments in this strand }\end{array}$ \\
\hline \multirow{2}{*}{$\begin{array}{l}\text { "1. Knowing, using, } \\
\text { and interpreting } \\
\text { scientific explanations } \\
\text { of the natural world" }\end{array}$} & \multirow{2}{*}{$\begin{array}{l}\text { Section: Student } \\
\text { Explanations or Explanatory } \\
\text { Models in the table }\end{array}$} & $\begin{array}{l}\text { Specify big ideas not lists of facts: Big ideas selected in } \\
\text { the progression are powerful explanatory ideas, not a } \\
\text { simple list of facts, that help learners explain important } \\
\text { aspects of the natural world. }\end{array}$ \\
\hline & & $\begin{array}{l}\text { Example learning performances in the progression reflect } \\
\text { interpreting and applying these important scientific ideas. } \\
\text { to explain phenomena (cluster } 1 \text { learning performances). }\end{array}$ \\
\hline $\begin{array}{l}\text { "2. Generating and } \\
\text { evaluating scientific } \\
\text { evidence and } \\
\text { explanations" }\end{array}$ & $\begin{array}{l}\text { Practices used in learning } \\
\text { performances }\end{array}$ & $\begin{array}{l}\text { Core ideas in the framework are not specified as } \\
\text { explanations to be consumed by learners. Instead, } \\
\text { learning performances make clear that the goals for } \\
\text { learning are the combination of both knowledge and } \\
\text { practice, not separate content and process learning goals. } \\
\text { The performances that represent strand 2, developing } \\
\text { scientific knowledge, are built using the investigation } \\
\text { cluster of practices (cluster } 2 \text { learning performances). }\end{array}$ \\
\hline \multirow{3}{*}{$\begin{array}{l}\text { "3. Understanding the } \\
\text { nature and } \\
\text { development of } \\
\text { scientific knowledge" }\end{array}$} & \multirow{3}{*}{$\begin{array}{l}\text { Practices used in learning } \\
\text { performances( but is implicit } \\
\text { and not explicit in the } \\
\text { learning performances) }\end{array}$} & $\begin{array}{l}\text { Practices are defined as meaningful engagement with } \\
\text { disciplinary practices, not rote procedures: }\end{array}$ \\
\hline & & $\begin{array}{l}\text { Practices are defined as meaningful practices, in which } \\
\text { learners are engaged in building, refining, and applying } \\
\text { scientific knowledge, to understand the natural world, and } \\
\text { not as rote procedures or a ritualized "scientific method." } \\
\text { Engaging in the practices requires being guided by } \\
\text { understandings about why scientific practices are done as } \\
\text { they are - what counts as a good explanation, what } \\
\text { counts as scientific evidence and how it differs from other } \\
\text { forms of evidence, and so on. These understandings are } \\
\text { represented in the nature of the practices. }\end{array}$ \\
\hline & & $\begin{array}{l}\text { We do not want to emphasize the nature of science as } \\
\text { decontextualized knowledge. Thus, we do not have } \\
\text { separate performances that solely refer to the nature of } \\
\text { science. Instead, we might have learning performances } \\
\text { that embed the use of these understandings in guiding the } \\
\text { practice, such as evaluating two candidate arguments and } \\
\text { analyzing their strengths and weaknesses. }\end{array}$ \\
\hline $\begin{array}{l}\text { "4. Participating } \\
\text { productively in } \\
\text { scientific practices and } \\
\text { discourse" }\end{array}$ & $\begin{array}{l}\text { Practices used in learning } \\
\text { performances }\end{array}$ & $\begin{array}{l}\text { Practices that require constructing arguments } \\
\text { Student must engage each other's ideas by critiquing, } \\
\text { defending, or justifying their explanation, models, data, or } \\
\text { designs of their investigations. We explicitly identify } \\
\text { learning performance that fall under as an indication that } \\
\text { students are engaged in this strand. }\end{array}$ \\
\hline
\end{tabular}




\section{Appendix C: \\ Relationship Between NRC Framework and Hypothetical Learning Progression for Structure, Properties \& Transformation of Matter}

\begin{tabular}{|c|c|}
\hline $\begin{array}{c}\text { Student Explanation/Explanatory Model in } \\
\text { Hypothetical Learning Progression (LP) }\end{array}$ & Content Statements \\
\hline $\begin{array}{l}\text { Level } 1 \text { (K-2) : Structure \& Properties } \\
\text { Level } 1 \text { Student Explanation/Explanatory Model } \\
\text { Focus Question: What is a material? } \\
\text { Different solid objects are made up of many different kinds of materials. Different } \\
\text { liquids are also made up of different materials. [Modified 1] Solid materials have } \\
\text { different properties (e.g., chalk, wax, and plastic differ in texture, color, flexibility, } \\
\text { and "squishiness" [malleability]). Liquid materials also have different properties } \\
\text { (e.g., liquid water, olive oil, and rubbing alcohol differ in taste, smell and ease of } \\
\text { flow [viscosity]). [Modified 2] Some materials can be found in nature; others are } \\
\text { synthetic (made by humans). Different materials are used to make objects with } \\
\text { different uses. [2] When you divide something into smaller pieces it is still the same } \\
\text { material (macroscopic compositional model). [Modified from } 3 \text { and 4] }\end{array}$ & $\begin{array}{l}\text { End of Grade } 2 \text { :Structure \& Properties } \\
\text { NRC Content Statements from PS1.A } \\
\begin{array}{l}\text { Matter exists as different substances (e.g., wood, metal, water), } \\
\text { and many of them can be either solid or liquid, depending on } \\
\text { temperature. [PS1.A, end of Grade 2] }\end{array} \\
\text { 2. Substances can be described and classified by their observable } \\
\text { properties (e.g., visual, aural, textural), by their uses, } \\
\text { and by whether they occur naturally or are manufactured. } \\
\text { Different properties are suited to different purposes. [PS1.A, end } \\
\text { of Grade 2] } \\
\text { 3. A great variety of objects can be built up from a small set of } \\
\text { pieces. [PS1.A, end of Grade 2] } \\
\text { 4. Objects or samples of a substance can be weighed and their } \\
\text { size can be described and measured [PS1.A, end of Grade 2] }\end{array}$ \\
\hline $\begin{array}{l}\text { Level } 1 \text { (K-2): Transformations from LP } \\
\text { Level } 1 \text { Student Explanation/Explanatory Model for Transformations } \\
\text { Focus Question: What happens to matter when it is physically remodeled? } \\
\text { If you cut up an object into many pieces its material does not change (for example, } \\
\text { if you cut up a chunk of wood into smaller pieces, its pieces are still wood). } \\
\text { [Modified from 2] The weight of all the pieces together is the same as the weight of } \\
\text { the object. [Modified from 3] Similarly, if you reshape an object made of clay or } \\
\text { wax (for example), it is still the same material and its weight stays the same. This is }\end{array}$ & $\begin{array}{l}\text { Grades 3-5: Transformations } \\
\text { NRC Content Statements from PS1.A } \\
\begin{array}{l}\text { 1. Objects or samples of a substance can be weighed and their } \\
\text { size can be described and measured [PS1.A, end of 2] }\end{array} \\
\text { 2. A great variety of objects can be built up from a small set of } \\
\text { pieces. [PS1.A, end of 2] }\end{array}$ \\
\hline
\end{tabular}




\section{Appendix C: \\ Relationship Between NRC Framework and Hypothetical Learning Progression for Structure, Properties \& Transformation of Matter}

\begin{tabular}{|c|c|}
\hline true for samples of liquids as well. [Added] & $\begin{array}{l}\text { 3. Objects or samples of a substance can be weighed and their } \\
\text { size can be described and measured [PS1.A, end of 2] }\end{array}$ \\
\hline $\begin{array}{l}\text { Level } 2 \text { (3-4): Structure \& Properties } \\
\text { Level } 2 \text { Student Explanation/Explanatory Model } \\
\text { Focus Question: What is matter? } \\
\text { Matter is anything that has weight and takes up space (i.e., has volume); types of } \\
\text { matter include solids and liquids. Matter can be imagined to exist in pieces too small } \\
\text { to see with the unaided eye, that still have weight, volume, and the same properties } \\
\text { as the material they compose. [5] One would need powerful microscopes and } \\
\text { sensitive scales to see these tiny pieces and measure their weight, but the model } \\
\text { posits that they would exist. [Added as clarification, but related to } 5 \text { and } 4 \text { ] } \\
\text { What are some of its states? } \\
\text { Matter exists as many different materials, many of which can transition back and } \\
\text { forth between solid and liquid. [1] } \\
\text { What are some Properties of Materials? } \\
\text { The temperature at which a material transitions from solid to liquid is characteristic } \\
\text { for each material. [1 and } 2 \text { (specific instance of a property)] } \\
\text { Objects made of some materials are heavier for the same volume than objects } \\
\text { made of other materials. [2 (specific instance of a property)] }\end{array}$ & $\begin{array}{l}\text { End of Grade } 2 \text { :Structure \& Properties } \\
\text { NRC Content Statements from PS1.A } \\
\text { 1. Matter exists as different substances (e.g., wood, metal, water), } \\
\text { and many of them can be either solid or liquid, depending on } \\
\text { temperature. [PS1.A, end of Grade 2] } \\
\text { 2. Substances can be described and classified by their observable } \\
\text { properties (e.g., visual, aural, textural), by their uses, } \\
\text { and by whether they occur naturally or are manufactured. } \\
\text { Different properties are suited to different purposes. [PS1.A, end } \\
\text { of Grade 2] } \\
\text { 3. A great variety of objects can be built up from a small set of } \\
\text { pieces. [PS1.A, end of Grade 2] } \\
\text { 4. Objects or samples of a substance can be weighed and their } \\
\text { size can be described and measured [PS1.A, end of Grade 2] } \\
\text { 5. Matter of any type can be subdivided into particles that are too } \\
\text { small to see, but even then the matter still exists and can be } \\
\text { detected by other means (e.g., by weighing or by its effects on } \\
\text { other objects). [PS1.A, end of Grade 5] } \\
\text { [Note, content statements targeted at the 3-5 grade band in the framework } \\
\text { include particles, but based on our interpretation of the research, students } \\
\text { in grades 3-4 are not ready for particles (M. Wiser, personal communication). } \\
\text { Therefore many of the content statements in the left column, our level 2, are } \\
\text { best aligned to the NRC content statements targeted at the end of Grade 2.] }\end{array}$ \\
\hline
\end{tabular}




\section{Appendix C: \\ Relationship Between NRC Framework and Hypothetical Learning Progression for Structure, Properties \& Transformation of Matter}

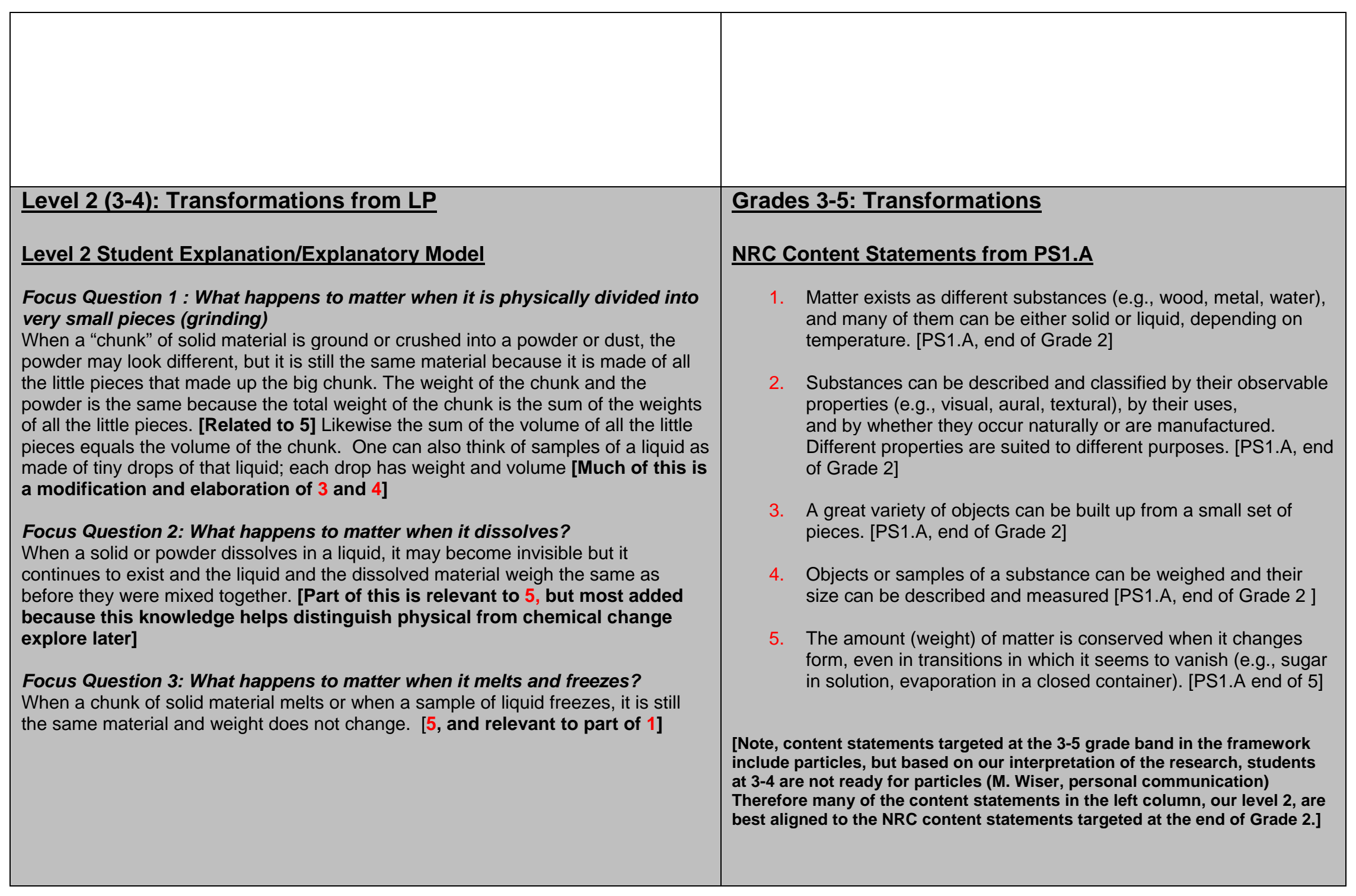




\section{Appendix C: \\ Relationship Between NRC Framework and Hypothetical Learning Progression for Structure, Properties \& Transformation of Matter}

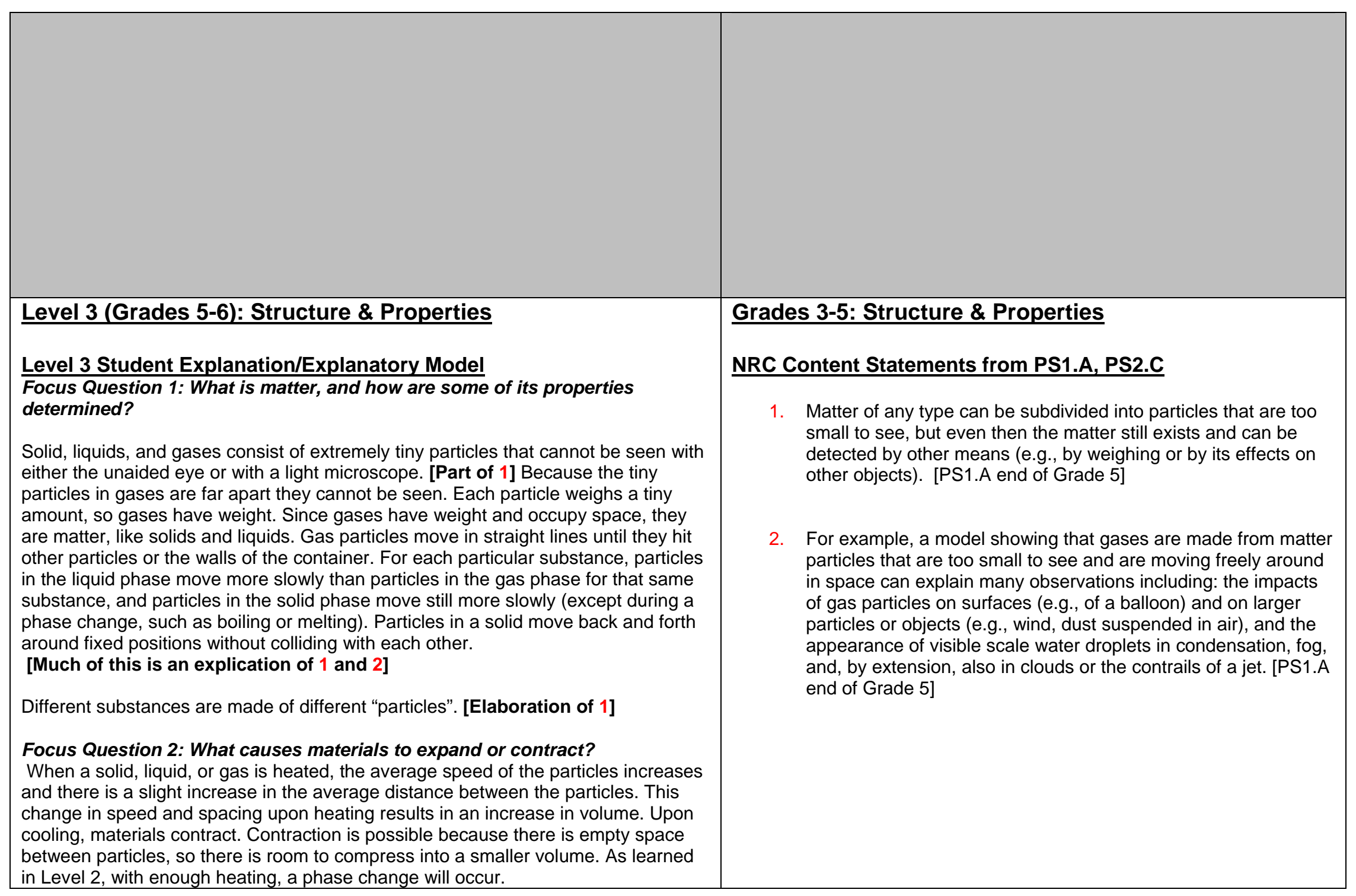




\section{Appendix C: \\ Relationship Between NRC Framework and Hypothetical Learning Progression for Structure, Properties \& Transformation of Matter}

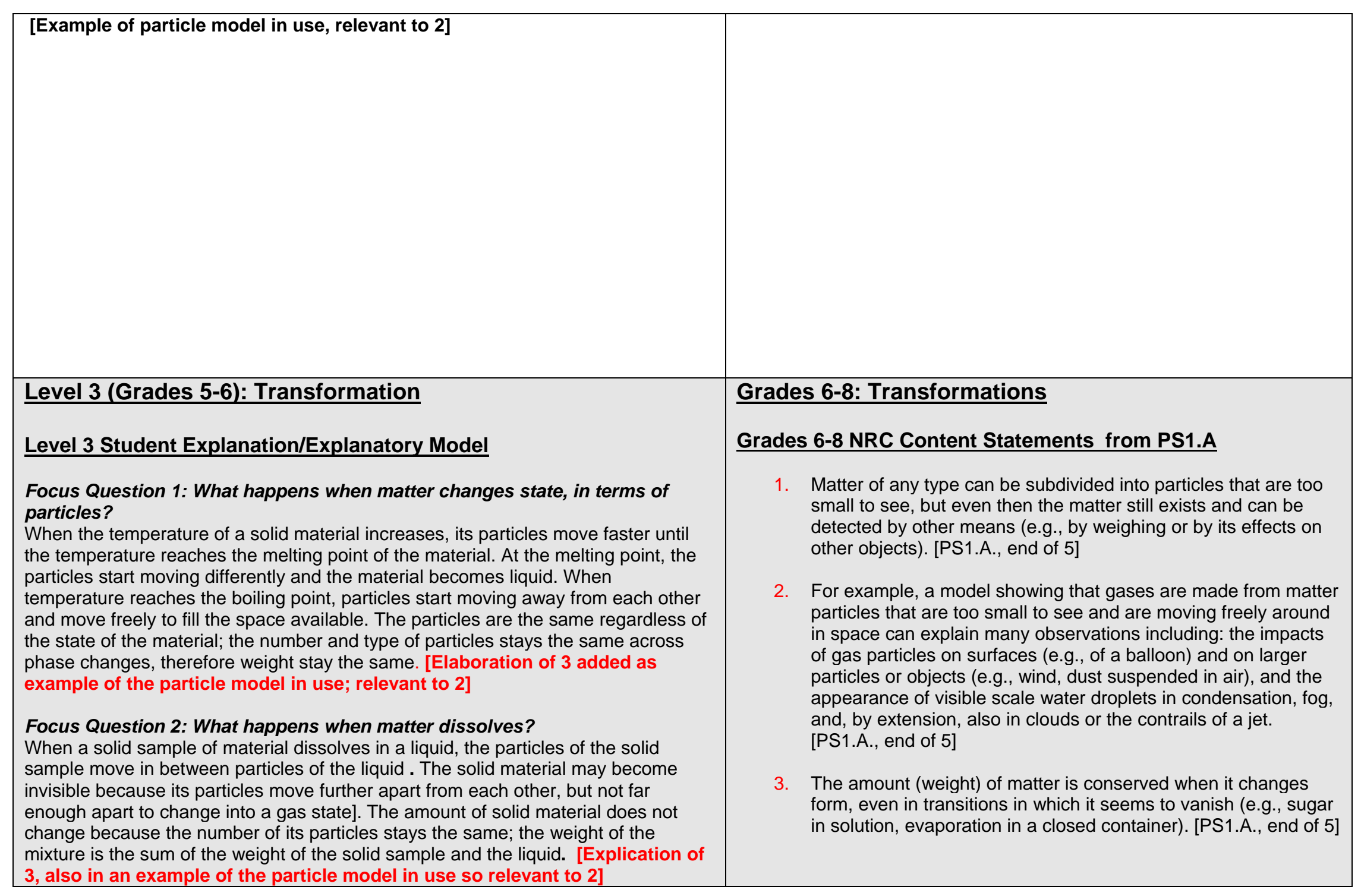




\section{Appendix C: \\ Relationship Between NRC Framework and Hypothetical Learning Progression for Structure, Properties \& Transformation of Matter}

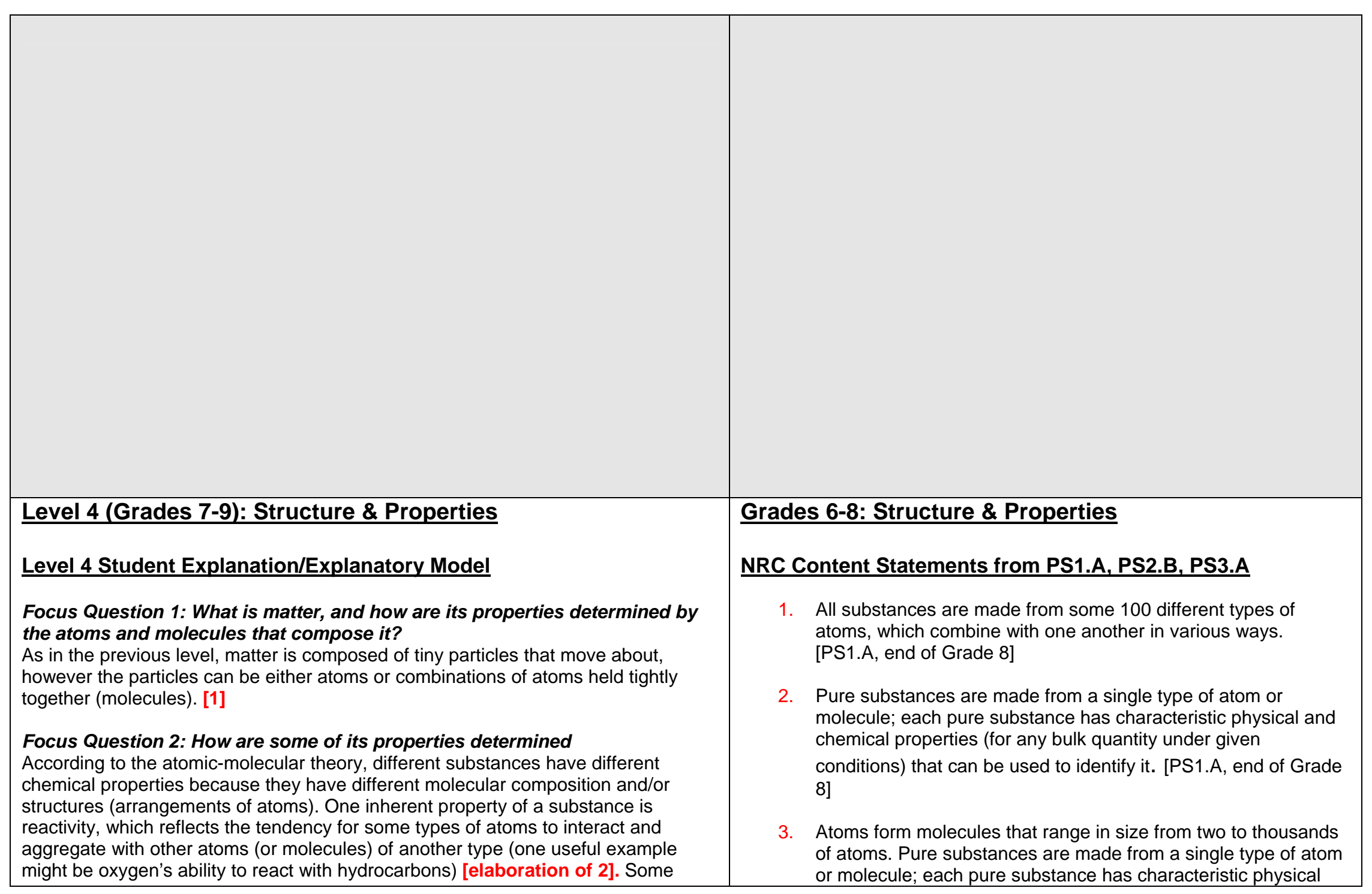




\section{Appendix C: \\ Relationship Between NRC Framework and Hypothetical Learning Progression for Structure, Properties \& Transformation of Matter}

substances have molecules made of the same type and same number of atoms; however, the arrangement of the atoms that make up the molecules is different. In this case, you have two different substances because they have different properties stemming from the different arrangement of atoms. [elaboration of 3] Related to this idea, the concepts of pure substances and mixtures can now be more adequately explained: a pure substance is made of the same type of atom or molecule throughout, while a mixture contains more than one type of atom or molecule throughout. [elaboration of 2]

\section{Focus Question 3: What are its states?}

When a substance is in its liquid state the attractive force between the molecules is weaker compared to when the same substance is in the solid state and therefore the molecules are not in fixed positions. Instead, in the liquid state, the particles can collide and slide past each other. Higher temperatures of a substance in the liquid state mean the molecules move more quickly and have more kinetic energy. When the same substance is in the gas state the attraction between its molecules is negligible, and can be ignored. In the gaseous state the molecules of the substance are moving faster than in the liquid state and have more kinetic energy. The molecules on average are very far apart in gases, compared to the distance between molecules in the solid or liquid state; molecules in a gas move in all directions, occasionally colliding with one another. Higher temperatures mean they move with greater speed and greater kinetic energy.

[Much of this in an elaboration of 4 and 5; ideas about attractive forces added but related to ideas found in 7 . Ideas about kinetic energy added but related

to ideas found in 8]

\section{Focus Question 4: How are some of its properties determined?}

Solids or liquids expand when heated and contract when cooled. In terms of forces, this happens because as the temperature rises the molecules move (or vibrate) with greater speeds. When this happens, the molecules tend to move further away from each other, weakening the strength of the attractive forces between the particles. As the temperature decreases, the particles move with lower speeds. When this happens the molecules tend to move closer together and the strength of the attractive forces between them increases. [Much of this in an elaboration of 4 and 5. Ideas about attractive forces added but related to ideas found in 7]

In terms of energy, when a solid or liquid material is heated (but not during melting or boiling), heat energy is transferred into the material, and this causes two things to happen. First, the average kinetic energy of the molecules increases, resulting in a rise in temperature. Second, the potential energy of the molecules increases as the molecules move a little further apart, and this results in the whole solid or liquid and chemical properties (for any bulk quantity under given conditions) that can be used to identify it. [PS1.A, end of Grade 8]

4. Gases and liquids are made of molecules or inert atoms that are moving about relative to each other. In a liquid, the molecules are constantly in contact with others; in a gas, they are widely spaced except when they happen to collide. In a solid, atoms are closely spaced and may vibrate in position but do not change relative locations. Solids may be formed from molecules, or they may be extended structures with repeating subunits (e.g., crystals). [PS1.A, end of Grade 8]

5. The changes of state that occur with variations in temperature or pressure can be described and predicted using these models of matter. [PS1.A, end of Grade 8]

6. Matter can be subdivided into particles... For example, a [particle] model showing that gases are made from matter particles that are too small to see and are moving freely around in space can explain many observations including: the impacts of gas particles on surfaces (e.g., of a balloon).... [PS1.A end of Grade 5]

7. Electric and magnetic (electromagnetic) forces can be attractive or repulsive, and their sizes depend on the magnitudes of the charges, currents, or magnetic strengths involved and on the distances between the interacting objects [PS2.B, end of Grade 8 interactions]

8. Motion energy is properly called kinetic energy; it is proportional to the mass of the moving object and grows with the square of its speed. A system of objects may also contain stored (potential) energy, depending on their relative positions. For example, energy is stored-in gravitational interaction with Earth-when an object is raised, and energy is released when the object falls or is lower. Energy is also stored 


\section{Appendix C: \\ Relationship Between NRC Framework and Hypothetical Learning Progression for Structure, Properties \& Transformation of Matter}

material expanding. [Students would need to recognize that as the distance between particles that attract each other increases; the potential energy of the system of particles increases- this would be a connection to the energy strand in the NRC document]. When a solid or liquid is cooled (but not during freezing or

condensation), heat energy is transferred from the system and this causes two things to happen. First, the average kinetic energy of the particles decreases, resulting in a lowering of temperature. Second, the potential energy of the particles decreases as the molecules move a little closer together, resulting in the whole solid or liquid material contracting. [Much of this in an elaboration of 4 and 5, ideas about attractive forces added but related to ideas found in 7 . Ideas about kinetic and potential energy added but related to ideas found in 8]

A gas exerts pressure on any surface it is in contact with because the molecules that are in constant motion push on the walls of the container when they collide with it. When the molecules move faster, they push harder on the walls and the pressure increases. Because the molecules move faster, they also collide more frequently with the walls of the container. This increase in the frequency of collision also accounts for increased pressure. The opposite happens when the temperature decreases. [Ideas about pressure exerted by gas is implied in part of 6; this is explicated]

The density of a substance is how much mass there is for each unit volume of the substance. This value does not depend on how much of a substance you have; thus it is known as a characteristic (or intrinsic) property and can help distinguish one substance from another. One substance has a higher density than another substance because its molecules have greater mass and/or because the molecules are on average closer together. [Elaborated on a physical property implied in 4]

\section{Level 4 (Grades 7-9): Transformations (Physical)}

\section{Level 4 Student Explanation/Explanatory Model}

Focus Question 1: What happens when matter changes states, in terms of atoms and molecules?

When a substance undergoes a physical change (like a change in state or an increase in volume as the temperature increases), the spacing of its molecules, the strength of the interaction between the molecules, and the kinetic energy and potential energy of the system all change. The molecules do not change, however. The total number of molecules (and thus, atoms) after the change is the same as in the electric fields between charged particles and the magnetic fields between magnets, and it changes when these objects are moved relative to one another..... [PS3.A, end of Grade 8, Energy]

\section{Grades 6-8: Transformations (Physical)}

\section{NRC Content Statements from PS1.A, PS2.B, PS3.A}

1. The changes of state that occur with variations in temperature or pressure can be described and predicted using these models of matter. [PS1.A, end of Grade 8]

2. [Matter can be subdivided into particles]... For example, a [particle] model showing that gases are made from matter 


\section{Appendix C: \\ Relationship Between NRC Framework and Hypothetical Learning Progression for Structure, Properties \& Transformation of Matter}

the total number before the change. Since each molecule has the same mass, this explains why the mass of a substance, composed of atoms or molecules, does not change during a physical change. [Much of this is an elaboration of 1]

\section{Focus Question 2: What happens during evaporation and condensation,} melting, and freezing?

At any given temperature molecules of a substance have a wide range of values of kinetic energies. Therefore, evaporation can occur at temperatures well below the boiling point because there are some molecules at the surface of liquids with sufficient kinetic energy to escape the attractive forces of neighboring molecules and escape into the gas state. Also, condensation can occur over a range of temperatures because there are some molecules in the gas phase with kinetic energies so low that they can't overcome the attractive forces from neighboring molecules in the liquid, and they too become molecules in the liquid state. [Much of this is an elaboration of 1 and 2 . Ideas about attractive forces added but related to ideas found in 9]

Each substance has a melting point because at a certain temperature the average kinetic energy of the molecules within a solid is sufficient to weaken the strength of the attractive forces between molecules, allowing the molecules to move past each other rather than remaining in fixed positions. Each substance has a unique melting point because the arrangement and strength of attraction between molecules is different for different substances. [Much of this is an elaboration of ideas related to 1, 3 (focus on specific physical property)]

Each substance has a boiling point because at a certain temperature the average kinetic energy of the molecules within a liquid is sufficient to overcome the attractive forces of nearby molecules, allowing the molecules to separate far from each other and become particles in the gas state. Each pure substance has a unique boiling point because the arrangement and strength of attraction between molecules is different for different substances. [Much of this is an elaboration of ideas related to $1,2,3]$

During both melting and boiling all the heat energy transferred into the material causes just the potential energy to increase; the average kinetic energy remains constant. This increase in potential energy reduces the attractive forces among the molecules. Thus, the temperature remains constant during melting or boiling. During melting, as the potential energy increases, the molecules break away from their fixed positions and begin to move past each other (and the material becomes a liquid). For some materials more heat energy is needed for the molecules to break particles that are too small to see and are moving freely around in space can explain many observations including: the impacts of gas particles on surfaces (e.g., of a balloon) and on larger particles or objects (e.g., wind, dust suspended in air), and the appearance of visible scale water droplets in condensation, fog..... [PS1.A end of Grade 5]

3. Pure substances are made from a single type of atom or molecule; each pure substance has characteristic physical and chemical properties (for any bulk quantity under given conditions) that can be used to identify it. [PS1.A, end of Grade 8]

9. Electric and magnetic (electromagnetic) forces can be attractive or repulsive, and their sizes depend on the magnitudes of the charges, currents, or magnetic strengths involved and on the distances between the interacting objects [PS2.B, end of Grade 8 interactions]

10. Motion energy is properly called kinetic energy; it is proportional to the mass of the moving object and grows with the square of its speed. A system of objects may also contain stored (potential) energy, depending on their relative positions. For example, energy is stored-in gravitational interaction with Earth-when an object is raised, and energy is released when the object falls or is lower. Energy is also stored in the electric fields between charged particles and the magnetic fields between magnets, and it changes when these objects are moved relative to one another..... [PS3.A, end of Grade 8, Energy] 


\section{Appendix C: \\ Relationship Between NRC Framework and Hypothetical Learning Progression for Structure, Properties \& Transformation of Matter}

\begin{tabular}{|c|c|}
\hline $\begin{array}{l}\text { the force of attraction among the particles than is needed for other materials; hence } \\
\text { they have a higher melting point. During boiling, as the potential energy increases } \\
\text { the molecules move very far from each other and travel in all directions (and the } \\
\text { material becomes a gas). For some materials the heat energy needed for this to } \\
\text { happen is greater than for other materials. [Much of this is an elaboration of } \\
\text { ideas related to } 1,2,3 \text {. Ideas about attractive forces added, but related to } \\
\text { ideas found in } 9 . \text { Ideas about energy added but related to ideas found in 10] }\end{array}$ & \\
\hline $\begin{array}{l}\text { Level } 4 \text { (Grades 7-9): Transformations (Chemical) } \\
\text { Level } 4 \text { Student Explanation/Explanatory Model } \\
\text { Focus Question: What happens to matter when substances change into new } \\
\text { substances? } \\
\text { When a chemical reaction occurs the atoms of the original substance rearrange or } \\
\text { regroup into different molecules that make up a new substance. The original and } \\
\text { new substances have different intrinsic properties. The total number and types of } \\
\text { atoms remain the same in a chemical reaction. This idea explains the conservation } \\
\text { of mass. [1 and 2] }\end{array}$ & $\begin{array}{l}\text { Grades 6-8: Transformations (Chemical) } \\
\text { NRC Content Statements from PS1.B } \\
\text { 11. Substances react chemically in characteristic ways. In a } \\
\text { chemical process, the atoms that make up the original } \\
\text { substances are regrouped into different molecules, and these } \\
\text { new substances have different properties from those of the } \\
\text { reactants. [PS1.B, end of Grade 8] } \\
\text { 12. The total number of each type of atom is conserved, and thus } \\
\text { the mass does not change. [PS1.B, end of Grade 8] }\end{array}$ \\
\hline
\end{tabular}




\section{Appendix C: \\ Relationship Between NRC Framework and Hypothetical Learning Progression for Structure, Properties \& Transformation of Matter}

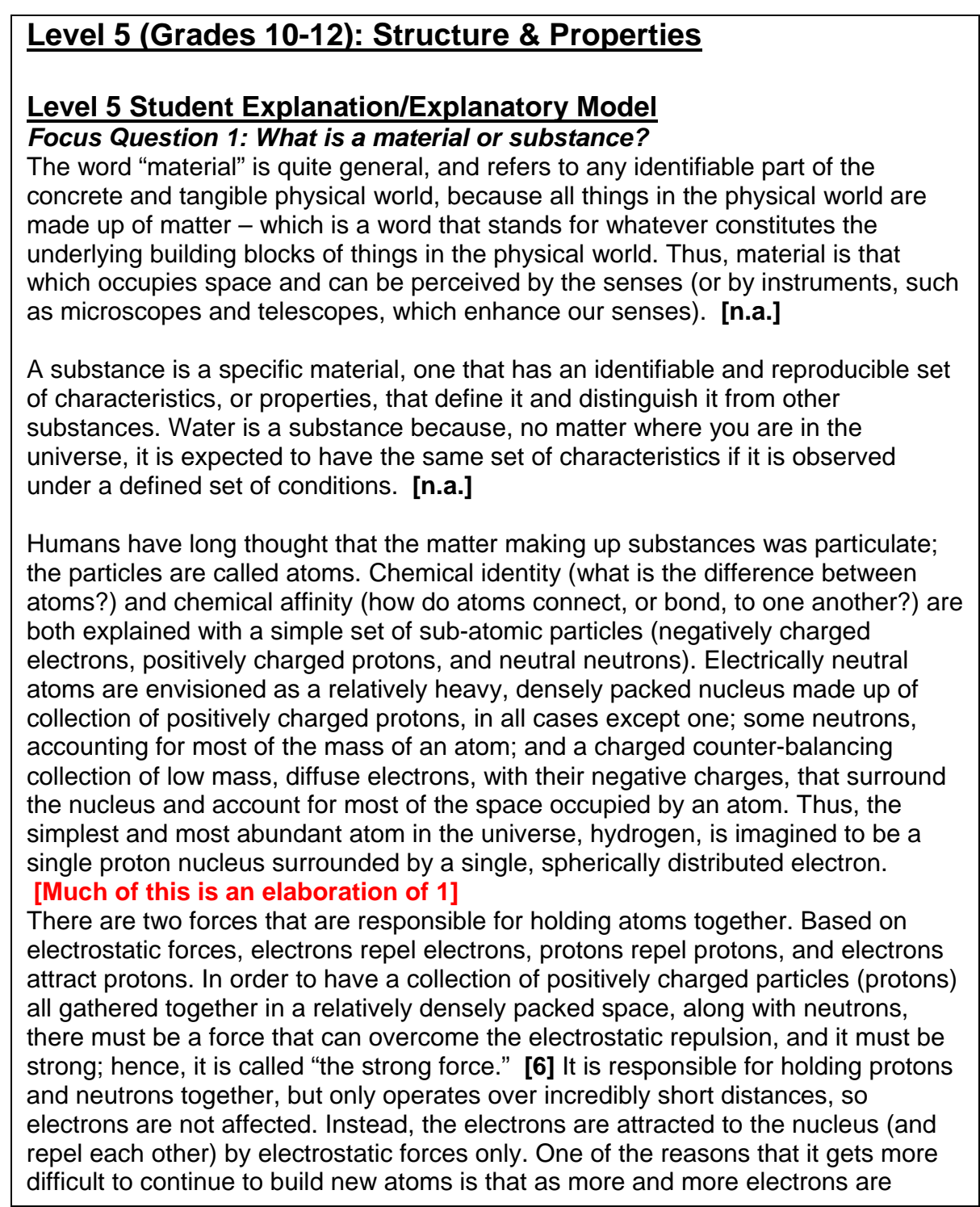

\section{Grades 9-12: Structure \& Properties \\ NRC Content Statements from PS1.A, PS2.B, PS1.C}

1. Each atom has a charged substructure consisting of a nucleus, which is made of protons and neutrons, surrounded by electrons. [PS1.A, end of Grade 12]

2. The periodic table orders elements horizontally by the number of protons in the atom's nucleus and places those with similar chemical properties in columns. The repeating patterns of this table reflect patterns of outer electron states. [PS1.A, end of Grade 12]

3. The structure and interactions of matter at the bulk scale are determined by electrical forces within and between atoms. [PS1.A, end of Grade 12]

4. Stable forms of matter are those in which the electric and magnetic field energy is minimized. [PS1.A, end of Grade 12]

5. Electric forces and magnetic forces are different aspects of a single electromagnetic interaction. Such forces can be attractive or repulsive, depending on the relative sign of the electric charges involved, the direction of current flow, and the orientation of magnets. The forces' magnitudes depend on the magnitudes of the charges, currents, and magnetic strengths as well as on the distances between the interacting objects..

Attraction and repulsion of electric charges at the atomic scale explain the structure, properties, and transformations of matter and the contact forces between material objects. [PS2.B, end of Grade 12]

6. The strong nuclear interaction provides the primary force that holds nuclei together and determines nuclear binding energies. Without it, the electromagnetic forces between protons would make all nuclei other than hydrogen unstable.... [PS1.C, end of 


\section{Appendix C: \\ Relationship Between NRC Framework and Hypothetical Learning Progression for Structure, Properties \& Transformation of Matter}

added to an atom, the amount of electron-electron repulsion increases, making the atoms larger and larger, and in turn causing the electrons to be farther and farther away from the attractive influence of the positively charged nucleus. Most of the large, artificial atoms have quite short lifetimes because there are not enough of the attractive forces to hold them together, and they quickly fall apart, sometimes after only a miniscule fraction of second.

Focus Question 2: What happens when atoms form molecules

The number of protons in a nucleus defines the chemical identity of an atom (for example, all one-proton atoms are hydrogen). One variety of atoms is isotopes, or atoms of the same element with a different number of neutrons. As one moves from hydrogen to helium to lithium to beryllium (chemical identity) on the periodic table, the number of protons increases from 1 to 2 to 3 to 4 , and so on. There are no gaps; presently, each member of the series from 1-118 nuclear protons has been identified. These are the chemical elements and are displayed in a table, called the periodic table, which is organized by properties of the elements' atomic structures. [Elaboration of 1 and part of 2]

Electrons occupy overlapping spherical shells of increasing radius. Each shell can hold a discrete number of electrons. The shell that fills first, which is closest to the nucleus, is called the $\mathrm{K}$ Shell and holds 2 electrons; L holds 8; M holds 18 , and so on. Atoms are most commonly uncharged, meaning the number of negatively

charged electrons associated with them matches the number of positively charged protons. A fundamentally important observation about atoms is that when a Shell is filled, that is, when the number of electrons equals the number that it can hold, the atom is relatively inert (does not easily form molecules) and generally exists as a mono-atomic entity. The exemplars are the inert gases-Helium, Neon, Argon,

Krypton, Xenon, and Radon-which sit in the far right column of the periodic table. Atoms like these are said to have closed-shell electron configurations.

[Much of this is an elaboration of part of 2 which reflects pattern in the outer shells of atoms]

When atoms are under conditions where they can become associated with one another, the closed shell electron arrangement provides a predictable way in which atoms can bond together. Individual hydrogen atoms (one proton, one electron) do not have a closed shell arrangement (i.e., 2 electrons in the $\mathrm{K}$ Shell). As 2 individual hydrogen atoms approach one another, a combination of electrostatic forces dictates what will happen: (1) the 2 positively charged nuclei repel each other; (2) the 2 negatively charged electrons repel each other; and (3) the protons and electrons are attracted. At some distance between the nuclei, the total sum of these forces reaches a minimum energy state, where the attractive forces are maximized 


\section{Appendix C: \\ Relationship Between NRC Framework and Hypothetical Learning Progression for Structure, Properties \& Transformation of Matter}

and the repulsive forces are minimized. At this distance, a bond has formed. The two nuclei are at a fixed distance. Both of the electrons are no longer spherically distributed around each individual nucleus, but are pictured as having formed an ellipsoid shape, covering the space of both nuclei, with a higher density of electrons along the internuclear axis (the imaginary axis that passes through the two nuclei in a bond), providing the maximum site for their attraction by the positive nuclei, while at the same time insulating the nuclei from their mutual repulsion of the positive protons because there are 2 electrons surrounding each of them, thus giving the appearance of a closed shell configuration to each nucleus in the diatomic (diatomic -being composed of 2 atoms) hydrogen molecule and providing a state of minimum energy. This is why hydrogen atoms combine (to achieve the closed shell

configuration that is at a minimum energy state) and why hydrogen molecules only involve 2 atoms of hydrogen. [Much of this is an elaboration that involves 1, 2 and 6. We added pieces about bonds, since other disciplines like the life sciences consistently refer to bonds]

Because each hydrogen atom achieves a closed shell configuration in the diatomic hydrogen molecule, the potential energy of the joined atoms (a molecule) is lower in the molecule than it was for each separate atoms. Energy, most probably in the form of thermal energy, would be released as the two hydrogen atoms bonded, sharing their electrons, and creating a more stable situation for the bonded

hydrogen atoms compared to when they were individual atoms. In order to separate the atoms in the hydrogen molecule, thermal energy (for example) would have to be added. [Part of this is relates to 4 , which relates to the with energy state of a molecule formed]

For some atoms, the shared arrangement is not the easiest way to achieve the closed shell configuration. Lithium has 3 electrons: 2 in the K Shell and 1 in the Shell. Electrons in an unfilled Shell are called valence electrons. In order to get to a closed shell configuration, lithium can lose an electron, thus becoming a positively charged atom, called an cation (3 protons/4 neutrons/2 electrons); otherwise, it would need to pack 7 more electrons around itself to get to the filled $L$ Shell of 8 , and this arrangement (3 protons/4 neutrons/8 electrons) would have lots of electron repulsion and not enough attractive force coming from the 3 protons to form a stable entity. Fluorine atoms, on the other hand, have just the opposite situation (9 protons $/ 10$ neutrons $/ 2 \mathrm{~K}$ electrons; $7 \mathrm{~L}$ electrons). In order to reach minimum energy of the closed $L$ Shell, fluorine needs 1 electron - just like the hydrogen atoms did in forming the hydrogen molecule. Thus, two fluorine atoms can form the diatomic fluorine molecule when they get within a close enough distance in order for a pair of electrons to be shared by both of the nuclei, thereby giving each nucleus the equivalent of a filled $L$ Shell. Alternatively, if fluorine atoms combine with lithium 


\title{
Appendix C: \\ Relationship Between NRC Framework and Hypothetical Learning Progression for Structure, Properties \& Transformation of Matter
}

\begin{abstract}
atoms, the fluorine atoms ( $2 \mathrm{~K}$ and $7 \mathrm{~L}$ electrons, could gain an electron to obtain the closed shell number, 8 ) can accept an electron from the lithium atoms $(2 \mathrm{~K}$ and $1 \mathrm{~L}$ electron, can get to the closest closed shell, $2 \mathrm{~K}$, by releasing an electron).

Consequently, lithium atoms (3 protons $/ 4$ neutrons/2K, $1 \mathrm{~L}$ electrons) will become a positively charged lithium cation ( 3 protons/4 neutrons/2K electrons) by the loss of an electron, while fluorine atoms (9 protons/10 neutrons/2K, $7 \mathrm{~L}$ electrons) will become the negatively charged fluoride anion ( 9 protons $/ 10$ neutrons $/ 2 \mathrm{~K}, 8 \mathrm{~L}$ electrons) by gaining an electron (anions are negatively charged atoms). Both atoms would reach a state of minimum energy. The resulting substance, lithium fluoride, is an ionic compound, made up of an equal, well-ordered collection of positive lithium cations and negative fluoride anions.

[Illustrative example of paragraphs above]

In all of these cases, the total number and kind of particles remains constant. There is no net build-up or loss of charge, and overall, in total, any sample of molecules will be uncharged.

Focus Question3 : why do some molecules have a charge or act like they have a charge (e.g. water and sugar molecules)

Because all particles must be conserved, the overall charge of any given chemical system is zero. Two uncharged hydrogen atoms (a pair of 1 proton/1 electron atoms) combine to give an uncharged hydrogen molecule ( 2 protons and 2 electrons). An uncharged lithium atom (3 protons, 4 neutrons, 3 electrons) and an uncharged fluorine atom (9 protons, 10 neutrons, 9 electrons) give a (positive)

lithium cation ( 3 protons, 4 neutrons, 2 electrons) and a (negative) fluoride anion (9 protons, 10 neutrons, 10 electrons). In this case, although each of the individual atoms carries a net charge, they are equal and opposite, involving a total of 12 protons, 14 neutrons, and 12 electrons in the system, both before and after) so that the net outcome is an uncharged system. Lithium cations and fluoride anions are called the counter-ions of an ionic compound ( $\mathrm{Li}+$, F-; lithium fluoride), while the two atoms in the diatomic hydrogen $(\mathrm{H}-\mathrm{H})$ or fluorine $(\mathrm{F}-\mathrm{F})$ molecules, in which the

electrons are being shared, are called covalent compounds. All of these atoms have closed shell electron configurations.

In the lithium fluoride example, each of the charged entities is just an atom with an imbalance of protons and electrons, resulting in a net charge at the atomic center. There are many examples of other, complex molecules that also carry one or more charges. In every case, however, the overall system will be uncharged because the total number of particles must remain conserved.
\end{abstract}




\section{Appendix C: \\ Relationship Between NRC Framework and Hypothetical Learning Progression for Structure, Properties \& Transformation of Matter}

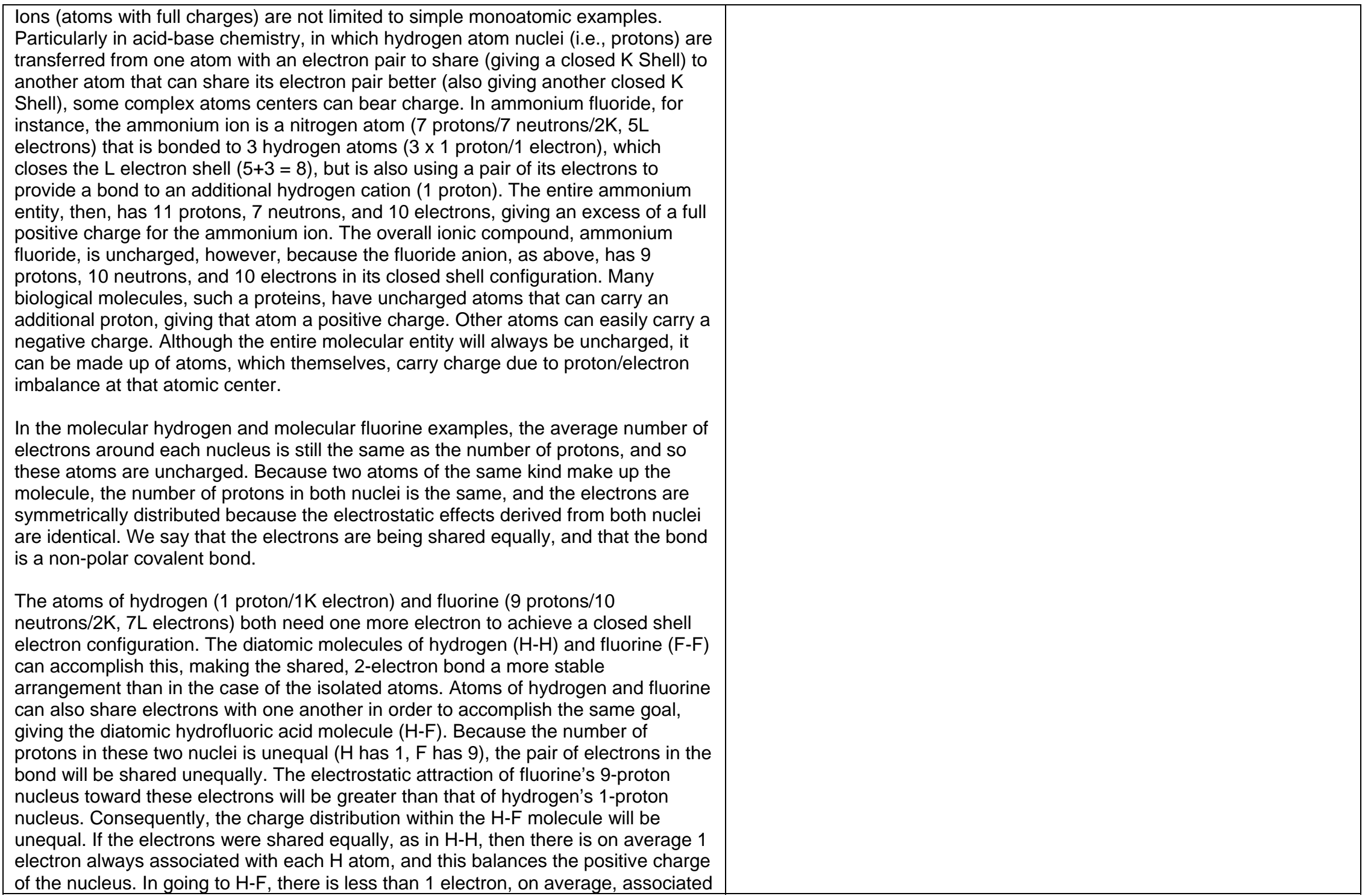




\section{Appendix C: \\ Relationship Between NRC Framework and Hypothetical Learning Progression for Structure, Properties \& Transformation of Matter}

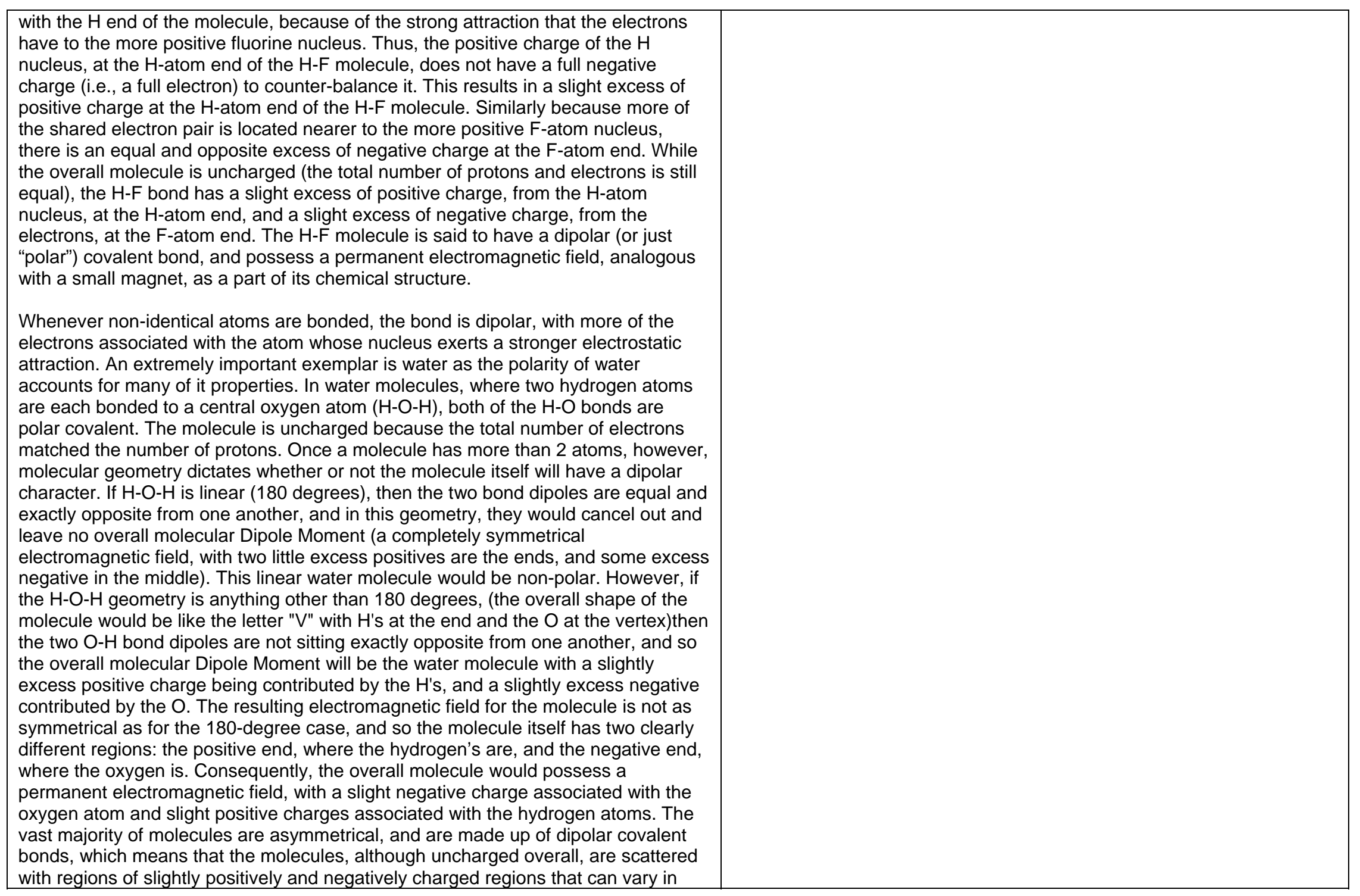




\section{Appendix C: \\ Relationship Between NRC Framework and Hypothetical Learning Progression for Structure, Properties \& Transformation of Matter}

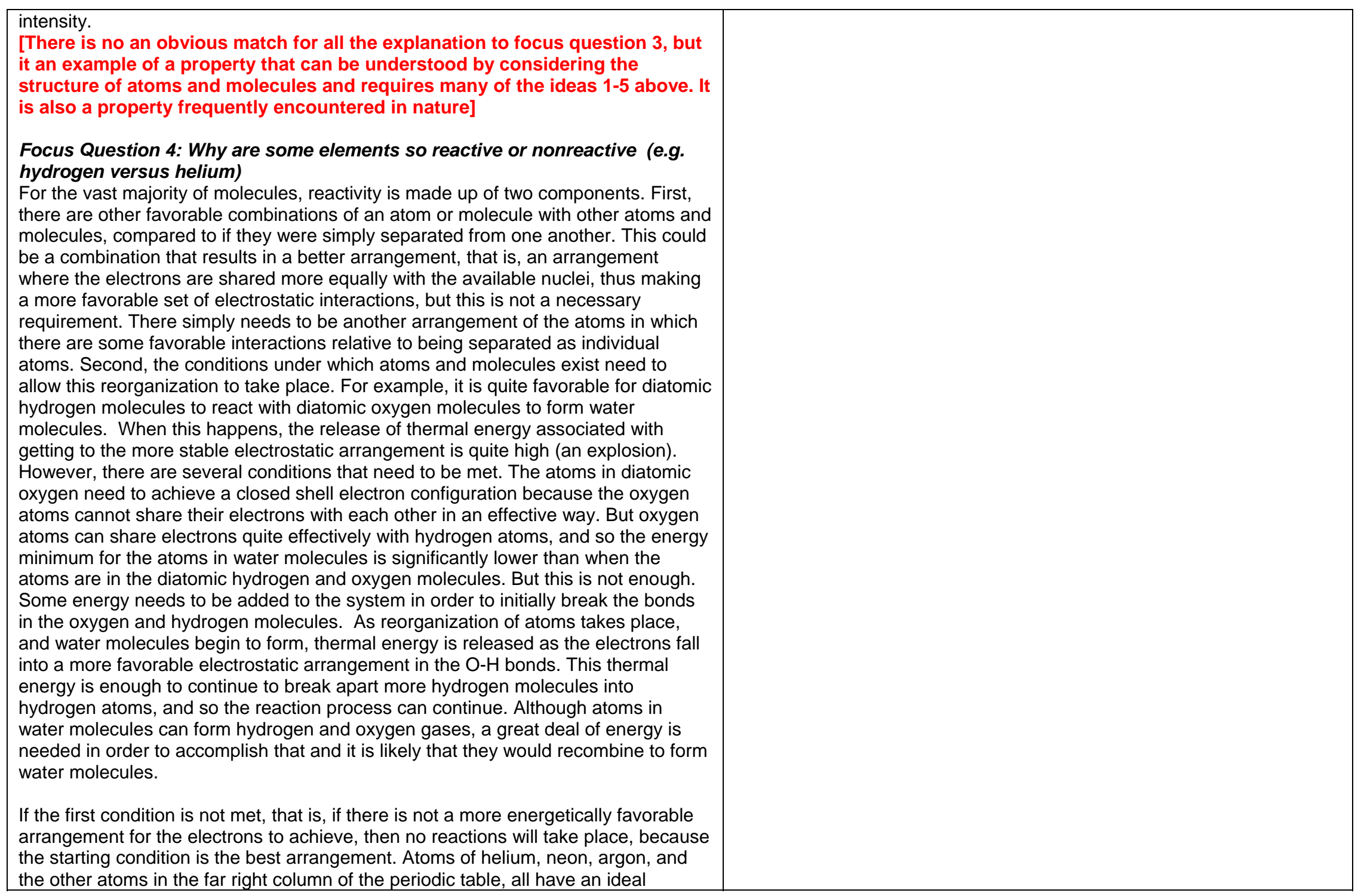




\section{Appendix C: \\ Relationship Between NRC Framework and Hypothetical Learning Progression for Structure, Properties \& Transformation of Matter}

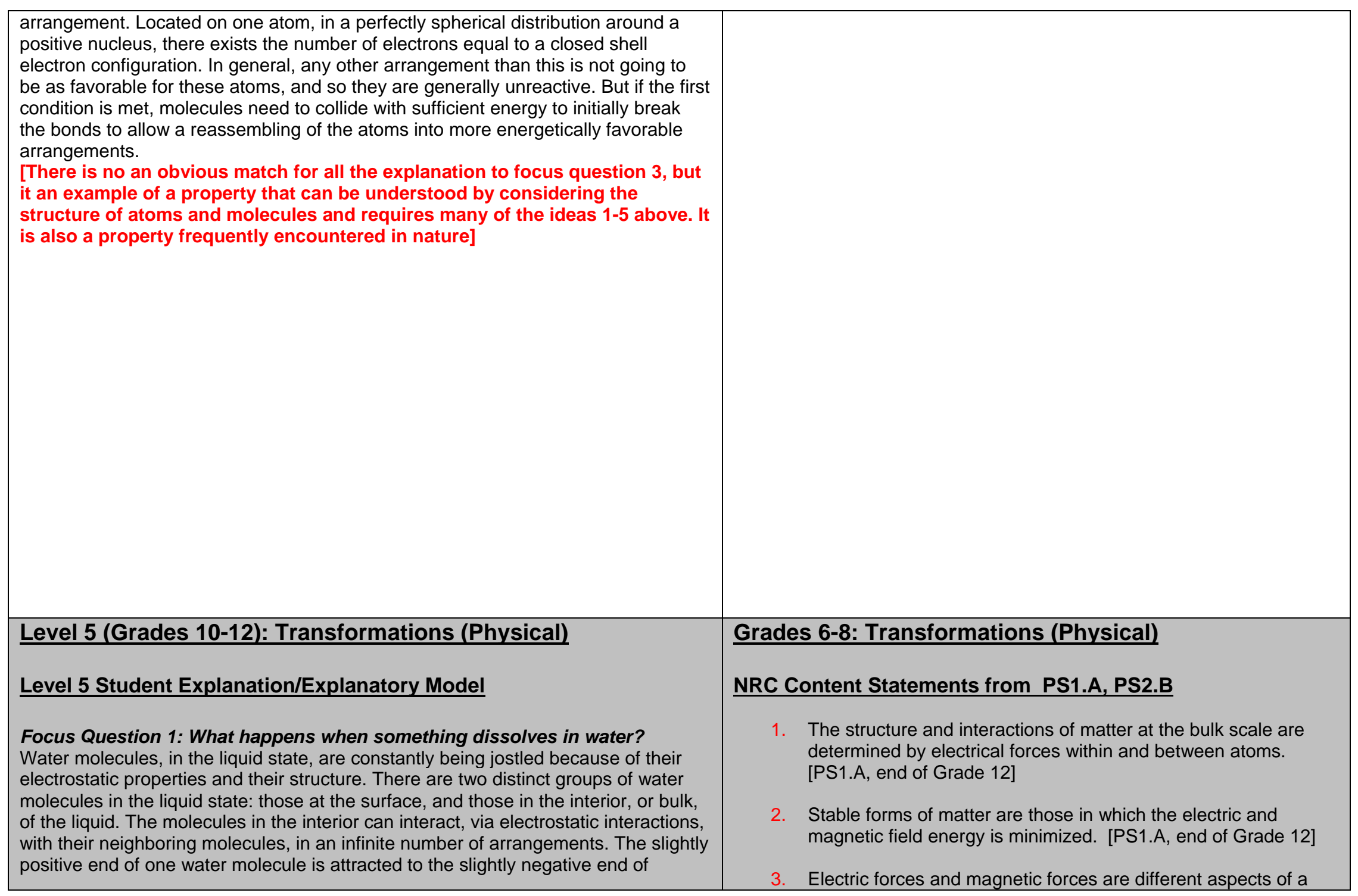




\section{Appendix C: \\ Relationship Between NRC Framework and Hypothetical Learning Progression for Structure, Properties \& Transformation of Matter}

another, and so on. And no matter how that molecule spins or moves, it finds itself in the presence of other partners with which it can be organized in a favorable electrostatic arrangement. The molecules at the surface of water, on the other hand, are not in a favorable electrostatic arrangement. In one hemisphere of space surrounding a surface water molecule (the water hemisphere) there are plenty of other water molecules to interact with, just as in the interior. But in the other hemisphere, perhaps this is a water droplet in the air, there are no other water molecules, just the vast openness of the open space where the sparse distribution of gas-phase molecules that make up the air are located. As a result, the water molecules on the surface of water are more highly organized and ordered than the ones in the interior in order to be able to receive the benefit of the electrostatic interaction with other water molecules. Water, therefore, behaves in a way to minimize its surface area, because the greater the surface of water, the more molecules there are that are not getting the energy benefit from electrostatic stabilization.

Dissolving means that two things are mixed and they stay mixed. Just as in a chemical reaction, the mixed state must be more stable than the unmixed state in order for a substance to dissolve in water. The interior of water, as described above, is quite favorable for water molecules because of the high number of electrostatic attractions that are possible. Thus, in order for a substance to dissolve in water, that substance needs to be able to replace, or at least compensate for, the electrostatic stabilization that water molecules have with one another. As a result, molecules that have the same kinds of charges and partial charges as water molecules can replace them in the bulk interior of water, and we say that they have dissolved, that is, they are intimately mixed with water molecules.

Most ionic compounds, because they are made up of fully charged ions that can interact well, electrostatically, with the polar ends of water molecules, can mix (dissolve) well. Molecules that are like water, and have a high fraction of polar covalent bonds in their structures, can also dissolve readily in water because the partial charges in those polar covalent bonds can provide favorable electrostatic interactions for the water molecules. Additionally, molecules in which $\mathrm{O}-\mathrm{H}$ and $\mathrm{N}-\mathrm{H}$ bonds dominate their chemical structure, readily dissolve in water, because these types of bonds are particularly polar (high partial charges).

If a molecule is not ionic, or it does not have polar covalent bonds, it generally does not dissolve in water. Gasoline, for instance, is made up of molecules with no polar covalent bonds, and it simply floats on top of water. Even when a water and gasoline mixture is agitated in order to try to get mixing to take place, the mixture will rapidly un-mix to give gasoline floating on the water again. When the molecules single electromagnetic interaction. Such forces can be attractive or repulsive, depending on the relative sign of the electric charges involved, the direction of current flow, and the orientation of magnets. The forces' magnitudes depend on the magnitudes of the charges, currents, and magnetic strengths as well as on the distances between the interacting objects..

Attraction and repulsion of electric charges at the atomic scale explain the structure, properties, and transformations of matter and the contact forces between material objects. [PS2.B, end of Grade 12] 


\section{Appendix C: \\ Relationship Between NRC Framework and Hypothetical Learning Progression for Structure, Properties \& Transformation of Matter}

in gasoline are forcibly inserted into the interior of water, those nearby water molecules lose some of the electrostatic stabilization they had when other water molecules were nearby. The water molecules near the molecules from gasoline are more organized and now have fewer electrostatic interactions. This is not favorable for the water molecules, which will be more stable when there are more sources of electrostatic interaction nearby. Consequently, the water and gasoline un-mixes, and this creates the least amount of surface area for the water molecules as possible.

[While the NRC framework does not address physical transformation directly, the explanation here draws from ideas in 1, 2, and 3. We included these explanations to show how the explanations become more sophisticated across time since there are explanations at the previous level that address the same phenomena. These are also commonly encountered phenomena]

Focus Question 2: What happens when something boils?

The molecules of a substance in the liquid state can move about, but are attracted to neighboring molecules through an electromagnetic interaction. The interaction arises because electrons in the atoms of one molecule are attracted to the positive charges in the atoms of neighboring molecules. As the temperature of the substance rises, the average kinetic energy of the molecules rises. Since the molecules have a range of kinetic energies, at any temperature those molecules at the surface with the greatest kinetic energies can overcome the attractive electromagnetic forces of the nearby molecules and escape into the space above the liquid surface. When this happens the molecules constitute the substance in the gas (vapor) state, and the process is called evaporation.

When the temperature becomes sufficiently high, molecules whose kinetic energy are at or above the average value have sufficient kinetic energy to escape the attractive electromagnetic forces of neighboring molecules throughout the entire liquid and escape into a space called a vapor bubble. Because the vapor bubbles have a much smaller density than the surrounding liquid they immediately rise to the surface. Molecules inside the bubble constitute the gas or vapor state of the substance. When this happens the process is called boiling, and the temperature at which it occurs is called the boiling point.

Another factor influencing boiling point is the mass of the atoms or molecules. For example, for non-polar molecules, the mass is the determining factor to explain boiling point. Heavier molecules have higher boiling points. 


\section{Appendix C: \\ Relationship Between NRC Framework and Hypothetical Learning Progression for Structure, Properties \& Transformation of Matter}

[While the NRC framework does not address physical transformation directly the explanation here draws from ideas in 1,2, and 3 . We included these explanations to show how the explanations become more sophisticated across time since there are explanations at the previous level that address the same phenomena. These are also commonly encountered phenomena]

\section{Level 5 (Grades 10-12): Transformations (Chemical)}

\section{Level 5 Student Explanation/Explanatory Model}

\section{Focus Question 1: What happen when a chemical reaction occurs?}

In order for a chemical reaction to occur, energy must be added to break the interactions that hold the atoms of the reactants together, thus increasing the potential energy of the separated atoms. In the reaction mixture, the starting molecules, or reactants, must collide with each other with the right orientation and enough kinetic energy in order to for the reaction to occur. When products are formed, atoms interact to reach a state with lower potential energy and energy is released in the process.

In a chemical reaction, molecular forms you begin with not only separate from one another, they also recombine into new forms as well as form back into the original structural arrangement. In chemical reactions, once energy is provided to move the atoms in the starting molecules away from their favorable bonding arrangements, there are generally many different options for how these atoms can recombine. It is rare when complex molecules undergo chemical reactions to give only one outcome; there are often many options for how the atoms might combine.

In complex molecules, not all of the atoms separate from one another. This would create a nearly infinite collection of molecular outcomes. Instead, it is generally the weakest bond, that is, the one with the least electrostatic stability in the molecule, which breaks. When the recombination of atoms and molecular pieces takes place, some of the resulting arrangements might be more stable and some might be less stable. Even a less stable arrangement can be the observed product from a chemical reaction because these new molecules form under conditions where, for instance, there is simply not enough energy around to break them back up. As a result, the outcome from a chemical reaction is not required to be the most stable arrangement of the atoms that is possible, only that arrangement which was least able to return to the reactive state of disconnected atoms. For instance, a less stable arrangement of atoms might precipitate from the solution, which might itself be a highly favorable process, and so these new molecules are simply not available for continued reaction. Another thing that might happen is that a new, less stable

\section{Grades 9-12: Transformations (Chemical) \\ NRC Content Statements from PS1.B, PS1.A, PS2.B}

1. Chemical processes, their rates, and whether or not energy is absorbed or released can be understood in terms of the collisions of molecules and the rearrangements of atoms into new molecules, with consequent changes in total binding energy (i.e., the sum of all bond energies in the set of molecules) that are matched by changes in kinetic energy. [PS1.B, end of Grade 12]

2. In many situations, a dynamic and condition-dependent balance between a reaction and the reverse reaction determines the numbers of all types of molecules present. [PS1.B, end of Grade 12]

3. Stable forms of matter are those in which the electric and magnetic field energy is minimized. [PS1.A, end of Grade 12] 


\section{Appendix C: \\ Relationship Between NRC Framework and Hypothetical Learning Progression for Structure, Properties \& Transformation of Matter}

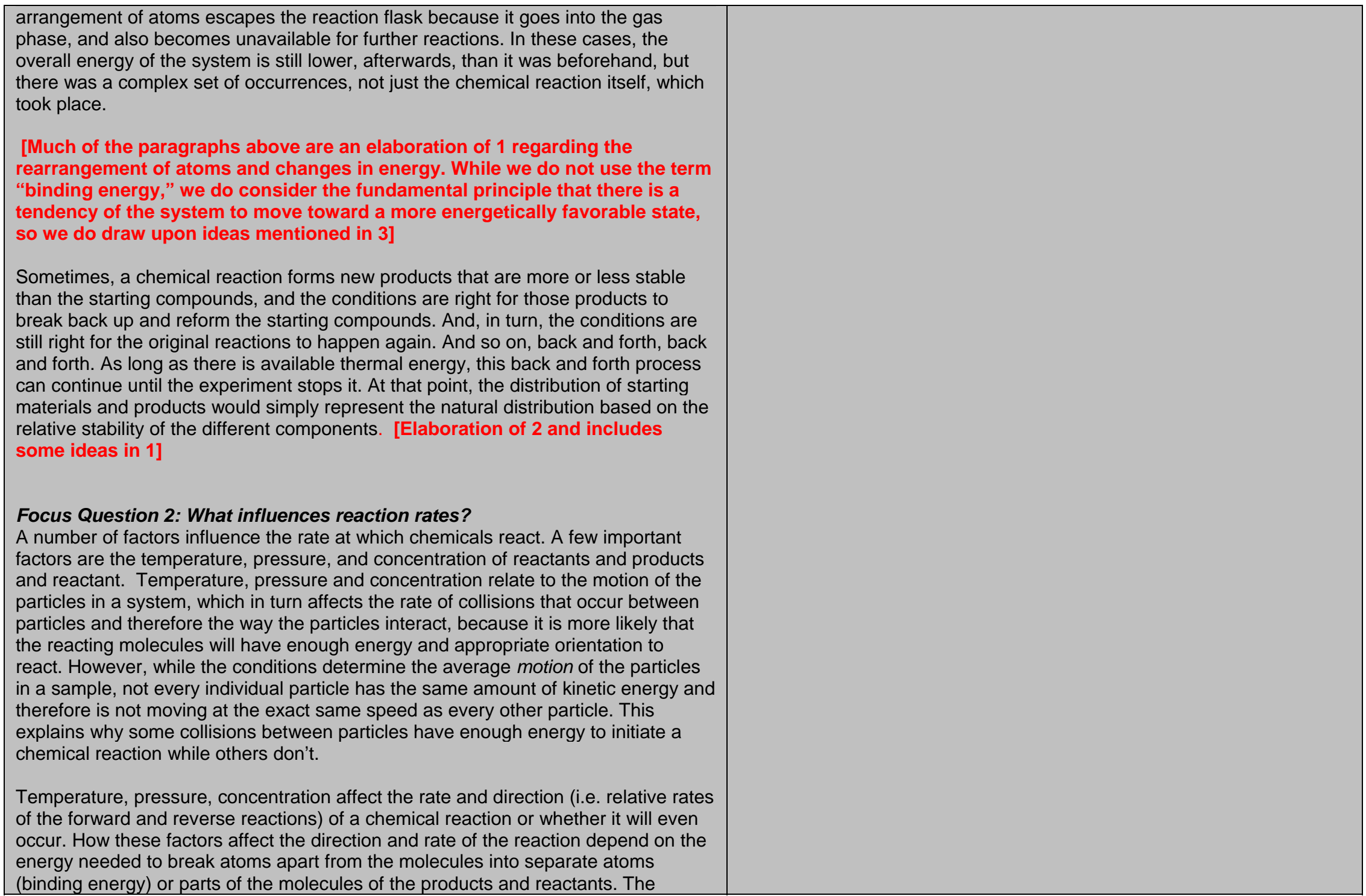




\section{Appendix C: \\ Relationship Between NRC Framework and Hypothetical Learning Progression for Structure, Properties \& Transformation of Matter}

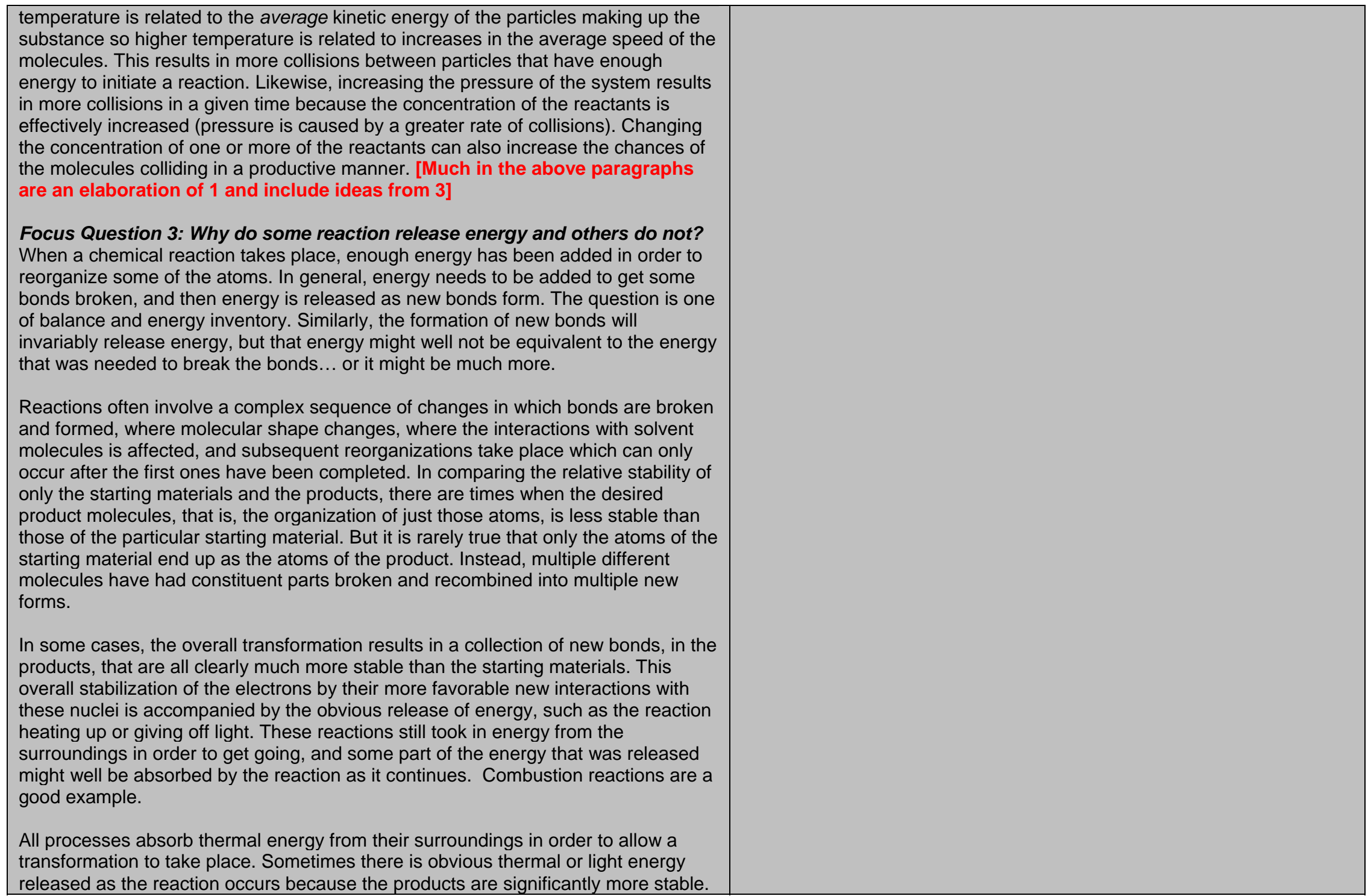




\section{Appendix C: \\ Relationship Between NRC Framework and Hypothetical Learning Progression for Structure, Properties \& Transformation of Matter}

\begin{tabular}{|l|l|}
\hline Combustion, and in the extreme, explosions, are a testimony to this. Other times, & \\
however, the main reaction products are less stable than the starting materials. Not \\
only is less thermal energy given off than is absorbed, but in the case where \\
product molecules go into the gas phase, even more energy can be absorbed from \\
the surroundings. In some of these cases, the reaction vessel might actually cool \\
down as thermal energy is pulled from the reaction system faster than it can \\
absorbed from the overall surroundings. \\
[Much in the above paragraphs are an elaboration of part 1 (reactions that \\
absorb or release energy) and include ideas from 3.]
\end{tabular}




\section{Appendix D: \\ Relationship Between NRC Framework and Hypothetical Learning Progression for the Flow of Matter \& Energy in Ecosystems}

\begin{tabular}{|c|c|}
\hline $\begin{array}{c}\text { Student Explanation/Explanatory Model in } \\
\text { Hypothetical Learning Progression (LP) }\end{array}$ & Content Statements \\
\hline Level 1 (K-2): Flow of Matter in Living Systems & $\begin{array}{l}\text { Grades K-2 : Matter \& Energy in Organisms and } \\
\text { Ecosystems }\end{array}$ \\
\hline $\begin{array}{l}\text { Student Explanation or Explanatory Model } \\
\text { Focus Question 1: What do organisms need to grow and survive? } \\
\text { Plants and animals are alive and grow. They both get things from their environment to } \\
\text { live and grow. [1] Observable growth signals life. As part of staying alive, living things do } \\
\text { certain things [biological work] - they grow, move, eat, and get rid of waste. To do these } \\
\text { things, animals get what they need by eating other living things or dead things.[2] They } \\
\text { also get what they need from non-living things (e.g., air and water).[added] }\end{array}$ & $\begin{array}{l}\text { NRC Content Statements for LS2.B and LS1.C } \\
\text { 1. Organisms obtain the materials they need to grow and survive } \\
\text { from the environment. Many of these materials come from } \\
\text { organisms and are used again by other organisms. [LS2.B] } \\
\text { 2. All animals need food in order to live and grow. They obtain } \\
\text { their food from plants or from other animals. [LS1.C] } \\
\text { 3. Plants need water and light to live and grow. [LS1.C] }\end{array}$ \\
\hline Plants need water and light, and do not eat other living things.[3] & \\
\hline Focus Question 2: What do organisms do with things they get from their & \\
\hline $\begin{array}{l}\text { environments? } \\
\text { There is some awareness of internal organs in animals to do something, especially } \\
\text { digestive systems.[added] }\end{array}$ & \\
\hline $\begin{array}{l}\text { Focus Question 3: How are organisms connected to each other and their } \\
\text { environments? } \\
\text { Animals consume plants and other animals. [2] }\end{array}$ & \\
\hline
\end{tabular}




\section{Appendix D: \\ Relationship Between NRC Framework and Hypothetical Learning Progression for the Flow of Matter \& Energy in Ecosystems}

\begin{tabular}{|c|c|}
\hline $\begin{array}{l}\text { Level } 2 \text { (Grades 3-5): Flow of Matter in Living Systems } \\
\text { Student Explanation or Explanatory Model } \\
\text { Focus Question 1: What do organisms need to grow and survive? } \\
\text { Animals need to ingest food to grow [although these transformations are understood } \\
\text { macroscopically and not at a cellular level.[3,4] Animals also need water, air, and food to } \\
\text { live, grow, and move. [2, 3] Water and air are not food. [added] } \\
\text { Plants need water, air, and sunlight to live and grow.[2, 3. 5] } \\
\text { Decomposers-macroscopic fungi and macroscopic invertebrates that facilitate decay- } \\
\text { are alive and grow as well as plants and animals. Decomposers and break down dead } \\
\text { plants and animals and return materials (nutrients) that plants can use to the soil.[added] } \\
\text { Focus Question 2: what do organisms do with things they get from their } \\
\text { environments? } \\
\text { Plants and animals both move materials around inside their bodies.[added] The material } \\
\text { is used for both growth and movement.[4] In animals, some food moves through the } \\
\text { digestive system and is eliminated as feces; other food reaches all parts of their bodies } \\
\text { and is used for growth.[6, modified] Decomposers feed on dead organic material. } \\
\text { [added] } \\
\text { Focus Question 3: how are organisms connected to each other and their } \\
\text { environments? } \\
\text { All animals eat plants or other animals, so all animals are connected to plants. This } \\
\text { connection is shown in a food web.[3] Plants need sunlight for growth.[3 from K-2] } \\
\text { Decomposers are part of the ecosystem too and feed off of dead plant or animal material. } \\
\text { [added] }\end{array}$ & $\begin{array}{l}\text { Grades 3-5: Matter \& Energy in Organisms and } \\
\text { Ecosystems } \\
\text { NRC Content Statements from LS1.C, LS2.B, LS1.A } \\
\text { 1. Matter cycles between the air and soil and among plants, } \\
\text { animals, and microbes as these organisms live and die. } \\
\text { [LS2.B] } \\
\text { 2. Organisms obtain gases, water, and minerals from the } \\
\text { environment and release waste matter (gas, liquid, or solid) } \\
\text { back into the environment. [LS2.B] } \\
\text { 3. Animals and plants alike generally need to take in air and } \\
\text { water, animals must take in food, and plants need light and } \\
\text { minerals. [LS1.C] } \\
\text { 4. Food provides animals with the materials they need for body } \\
\text { repair and growth and is digested to release the energy they } \\
\text { neeed to maintain body warmth and for motion. LS1.C] } \\
\text { 5. Plants acquire their material for growth chiefly from air and } \\
\text { water and process matter they have formed to maintain their } \\
\text { internal conditions (e.g., at night). [LS1.C] } \\
\text { 6. Plants and animals have both internal and external structures } \\
\text { that serve various functions in growth, survival, behavior, and } \\
\text { reproduction. [LS1.A] }\end{array}$ \\
\hline $\begin{array}{l}\text { Level } 3 \text { (Grades 6-8): Flow of Matter \& Energy in Living Systems } \\
\text { Level } 3 \text { Student Explanation or Explanatory Model } \\
\text { Focus Question 1: What do organisms need to grow and survive? } \\
\text { Plants, animals, and decomposers obtain matter and energy that they need to live, }\end{array}$ & $\begin{array}{l}\text { Grades 6-8: Matter \& Energy in Organisms and } \\
\text { Ecosystems } \\
\text { NRC Content Statements from LS2.B, LS1.C,LS1.A }\end{array}$ \\
\hline
\end{tabular}




\section{Appendix D: \\ Relationship Between NRC Framework and Hypothetical Learning Progression for the Flow of Matter \& Energy in Ecosystems}

function, and grow from their environments. Decomposers include microscopic bacteria and fungi, which also need matter to grow and function. [4]

Animals and decomposers are different from plants in that they must consume food molecules (e.g. sugar) from other organisms (living or dead). Plants are unique in that they use the energy from sunlight to make sugars (food) from carbon dioxide and water. [4] This process transforms light energy from the sun into stored chemical energy. Food molecules in plants and animals are both used in the same way, they a) react with oxygen to provide energy that is needed to carry out life functions, b) build and become incorporated into the body structure, or c) are stored for later use.[5]

\section{Focus Question 2: What do organisms do with things they get from their}

\section{environments?}

Plants, animals, and decomposers are all made of cells, which carry out life functions.

[8\&9] Plant cells rearrange the atoms in carbon dioxide and water molecules into sugar and oxygen molecules.[4] Sugars contain stored chemical energy. [added] Minerals and other nutrients from the soil are not food (they don't provide energy), but plants combine them with sugar to make complex molecules that make up plant bodies and enable them to function. This is how plants grow.

Animals and decomposers grow by consuming complex molecules, distributing them to al parts of their bodies, and modifying them into other complex molecules. In animals food molecules are broken down in the digestive system and the resulting smaller molecules are transported to all the other cells of the body by the circulatory system where they can be incorporated into cell structures when growth occurs.[6,7 had digestive system and circulatory system in at this grade band in early drafts of framework]

Plants, animals, and decomposers. Inside the cells of these organisms oxygen reacts with foodstuffs (such as sugars) to produce carbon dioxide and water in a process called cellular respiration. [7] Thus food molecules go through a transformation in which atoms the make them up are rearranged to form new substances. When food reacts with oxygen it serves as a source of energy because the reaction with oxygen releases energy that the cell can use to drive growth or functioning (like movement in animals).

\section{Focus Question 3: How are organisms connected to each other and their} environments?

Matter: Matter is transferred between different organisms in an ecosystem. Plants get carbon dioxide from the atmosphere, which can come from animals and decomposers,
1. Food webs are models that demonstrate how matter and energy is transferred between producers (generally plants and other organisms that engage in photosynthesis), consumers, and decomposers as the three groups interact-primarily for food- within an ecosystem. [LS2.B]

2. Transfers of matter into and out of the physical environment occur at every level, for example when molecules from food react with oxygen captured from the environment, the carbon dioxide and water thus produced are transferred back to the environment, and ultimately so are waste products, such as fecal material. [LS2.B]

3. Decomposers recycle nutrients from dead plant or animal matter back to the soil in terrestrial environments or to the water in aquatic environments. The atoms that make up the organ isms in an ecosystem are cycled repeatedly between the living and nonliving parts of the ecosystem. [LS2.B]

4. Plants, algae (including phytoplankton), and many microorganisms use the energy from light to make sugars (food) from carbon dioxide from the atmosphere and water through the process of photosynthesis, which also releases oxygen. [L1.C]

5. These sugars can be used immediately or stored for growth or later use. [L1.C]

6. Animals obtain food from eating plants or eating other animals. Within individual organisms, food moves through a series of chemical reactions in which it is broken down and rearranged to form new molecules, to support growth, or to release energy. [L1.C]

7. In most animals and plants, oxygen reacts with carbon containing molecules (sugars) to provide energy and produce waste carbon dioxide; anaerobic bacteria achieve their energy needs in other chemical processes that do not require oxygen. [L1.C]

8. Unicellular organisms (microorganisms), like multicellular organisms, need food, water, a way to dispose of waste, and an environment in which they can live. [LS1.A]

9. In multicellular organisms, the body is a system of multiple interacting subsystems and groups of cells that work together to form tissues and organs that are specialized for particular 


\section{Appendix D: \\ Relationship Between NRC Framework and Hypothetical Learning Progression for the Flow of Matter \& Energy in Ecosystems}

\begin{tabular}{|c|c|}
\hline $\begin{array}{l}\text { and transform this compound into food stuff that plants can use. Animals get food from } \\
\text { eating plants of other animals as described in food webs. Decomposers get food from } \\
\text { dead animals or plants found in their environment and break down matter found in this } \\
\text { dead matter to generate feely available minerals that plants can absorb from the soil (and } \\
\text { therefore help to recycle matter to plants). [elaboration of } 1 \text { and 3] } \\
\text { Energy: Energy flows through ecosystems: sunlight is converted to chemical energy } \\
\text { through photosynthesis in plants. That chemical energy is stored in plant bodies and } \\
\text { passed on to animals and decomposers. Plants, animals, and decomposers all use } \\
\text { stored chemical energy for growth and other organism functions; in particular animals use } \\
\text { the chemical energy for necessary functions such as movement. [n elaboration of } 7 \text { and } \\
\text { related to } 1 \text { and 2] }\end{array}$ & body functions. [LS1.A] \\
\hline $\begin{array}{l}\text { Level } 4 \text { (Grades 9-12): Flow of Matter \& Energy in Living Systems } \\
\text { Level } 4 \text { Student Explanation or Explanatory Model }\end{array}$ & $\begin{array}{l}\text { Grades 9-12: Matter \& Energy in Organisms and } \\
\text { Ecosystems } \\
\text { NRC Content Statements from LS2.B.LS1.C, LS1.A }\end{array}$ \\
\hline $\begin{array}{l}\text { Focus Question 1: what do organisms need to grow and survive? } \\
\text { As a general rule, as matter cycles and energy flows through different levels of } \\
\text { organization of living systems, and between living systems and the physical environment, } \\
\text { matter and energy are conserved in each change.[part of } 4 \text { and 10] } \\
\text { All organisms are made of the same basic materials, including water, and particular } \\
\text { biological molecules such as carbohydrates, lipids, nucleic acids (i.e. DNA) and proteins } \\
\text { (all have carbon as their basic building block). [added but is related to and is an } \\
\text { elaboration of 11] As a result all organisms need to get the same substances to support } \\
\text { their growth. These substances can be found in food.[added] Animals and decomposers } \\
\text { consume these materials in the food they eat. Plants are unique in their ability to capture } \\
\text { energy from sunlight and use it to construct these materials from inorganic ingredients: } \\
\text { carbon dioxide, water, and soil minerals. [6] } \\
\text { Focus Question 2: what do organisms do with things they get from their } \\
\text { environments? } \\
\text { As a general rule, the growth and functioning of all organisms occurs though chemical } \\
\text { processes in which atoms are rearranged into new molecules inside cells. These } \\
\text { chemical reactions involve the transformation of matter (in which atoms rearrange to form } \\
\text { different substances). [7] These chemical reactions also involved the transfer of energy } \\
\text { (from one form of energy to another, or from one system of interacting molecules to } \\
\text { another). Both matter and energy are conserved during transfer. [8] }\end{array}$ & $\begin{array}{l}\text { 1. Photosynthesis and cellular respiration (including anaerobic } \\
\text { processes) provide most of the energy for life processes. } \\
\text { Plants or algae form the lowest level of the food web. [LS2.B] } \\
\text { 2. Only a small fraction of the matter consumed at the lower level } \\
\text { in a food web is transferred upward } \\
\text { 3. The chemical elements that make up the molecules of } \\
\text { organisms pass through food webs and into and out of the } \\
\text { atmosphere and soil, and are combined and recombined in } \\
\text { different ways. [LS2.B] } \\
\text { 4. At each link in an ecosystem, matter and energy are } \\
\text { conserved; some matter reacts to release energy for life } \\
\text { functions, some matter is stored in newly made structures, and } \\
\text { much is discarded. [LS2.B] } \\
\text { Photosynthesis and cellular respiration are important } \\
\text { components of the carbon cycle, in which carbon is exchanged } \\
\text { between the biosphere, atmosphere, oceans, and geosphere } \\
\text { through chemical, physical, geological, and biological } \\
\text { processes.[LS2.B] } \\
\text { The process of photosynthesis converts light energy to stored } \\
\text { chemical energy by converting carbon dioxide plus water into } \\
\text { sugars plus released oxygen. The sugar molecules thus }\end{array}$ \\
\hline
\end{tabular}




\section{Appendix D: \\ Relationship Between NRC Framework and Hypothetical Learning Progression for the Flow of Matter \& Energy in Ecosystems}

Matter: Plants utilize compounds from sugar generated through photosynthesis along with soil minerals to make amino acids and other carbon-containing molecules. These molecules can then be assembled into larger molecules like complex carbohydrates, lipids, nucleic acids, and proteins (biosynthesis) that can either be incorporated into plant structures or perform biological activity (e.g. enzymes) inside of plants. Animals and decomposers grow by digesting carbohydrates, lipids, nucleic acids and proteins (i.e., breaking them down into smaller organic molecules) and assembling them into new larger biological molecules (biosynthesis) that can then be incorporated into animal structures or perform biological activity inside of animals. Therefore, all organisms engage in biosynthetic chemical reactions to support growth and functioning.[elaboration of 6 from Grade 6-8] Therefore, all organisms engage in biosynthetic chemical reactions to support growth and functioning

Energy: Through photosynthesis, plants take energy from light to form sugar molecules (foodstuffs) containing carbon, hydrogen, and oxygen. Photosynthesis and the production of sugars occur in specialized sub-cellular locations/structures [chloroplasts] that are only found in plants [and other photosynthetic micro-organisms]. During this process water and carbon dioxide react to form sugar and oxygen.[much of this is related to and is an elaboration of 1 , and draw upon 11]

Through cellular respiration, which takes place in most organisms, including animals, plants, and most decomposers, a form of energy is generated that drives the chemical reactions that support growth and functioning. In this cellular process, oxygen reacts with foodstuffs (i.e., carbohydrates, lipids, nucleic acids and proteins) that form carbon dioxide and water. [8] During this cellular process energy is transferred from one set of molecules to another set of molecules [e.g. ATP] as bonds are broken and reformed during chemical reactions that take place in special subcellular locations or structures inside of the cells in plants, animals or decomposers [e.g. mitochondria]. [elaboration of 8 and draws upon 11] This reaction, like the burning of wood or coal, involves oxygen, is highly controlled and energy is transferred to other molecules that can be transported to cells all over the organism to drive other chemical reactions. These chemical reactions support cellular processes such as biosynthesis to support growth or reproduction or movement of molecules within and across cells to support cellular functioning. Biological work can include not only movement but the synthesis of large macromolecules such as protein or DNA needed during growth. Since principles of energy conservation apply to living systems, inputs of energy are always equal to energy that is stored, used, released (as heat), or excreted from organisms. Thus any change in input must be accounted for by changes in energy stored, used, released, or excreted-or vice versa. formed contain carbon, hydrogen, and oxygen, and some trace minerals that are used to make amino acids and othe carbon-based molecules that can be assembled into larger molecules (such as proteins or DNA), used for example to form new cells. [LS1.C]

7. As matter and energy flow through different organizational levels of living systems, chemical elements are recombined in different ways to form different products.

8. As a result of these chemical reactions energy is transferred from one system of interacting molecules to another. For example, aerobic (in the presence of oxygen) cellular respiration is a chemical process in which the bonds of food molecules and oxygen molecules are broken and new compounds are formed that can transport energy to muscles. [LS1.C]

9. Cellular respiration also releases the energy needed to maintain body temperature despite ongoing energy loss to the surrounding environment. [LS1.C]

10. Matter and energy are conserved in each change. This is true of all biological systems, from individual cells to ecosystems. [LS1.C]

11. Multicellular organisms have a hierarchical structural organization, in which any one system is made up of numerous parts and is itself a component of the next level. [LS1.A] 


\section{Appendix D: \\ Relationship Between NRC Framework and Hypothetical Learning Progression for the Flow of Matter \& Energy in Ecosystems}

\section{Focus Question 3: How are organisms connected to each other and their}

environments?

In general: All processes that occur at the cellular, organismal, and population scales are part of the general processes of energy flow and carbon cycling in ecosystems and principles of conservation of matter and energy apply throughout every part of the ecosystem.

Matter Flow (Carbon Cycling): Carbon (and other key elements used by biological organisms such as phosphorus and nitrogen) is recycled within the ecosystem. The chemical elements that make up the molecules of organisms pass through food webs and are combined and recombined in different ways. Carbon is transformed from carbon dioxide and water into the carbohydrates, lipids, nucleic acids and proteins that make up living organisms through processes that occur in organisms such as photosynthesis, digestion, and biosynthesis, and is subsequently returned (recycled) back to the atmosphere as carbon dioxide through cellular respiration which occurs in all organisms. Only a small fraction of the matter consumed at the lower level of a food web is transferred upward. [2, much of this is an elaboration of 1 from Grades 3-5 and 1 from Grades 6-8 with emphasis on carbon]

Energy Flow: Energy flows through the ecosystem moving from one place to another. Some of it is used at each step to support life activities, and typically at each transfer some is released as heat into the environment- it is not recycled like matter (however energy conservation principles still apply). Energy flow begins with plants where it is transferred through cellular processes such as photosynthesis, cellular respiration, biosynthesis, and digestion to support growth and other life functions. Some energy then flows to animals where it is transferred through cellular processes such as digestion, cellular respiration, and biosynthesis to support growth and function. Organic matter found in dead organisms or waste products from living organisms can flow to decomposers where similar processes of digestion, cellular respiration, and biosynthesis can occur. Since, only photosynthetic organisms like plants or some algae can produce their own food using energy from the sun, and other organisms eat those plants or consume other animals that ate the plants, all matter in most ecosystems can be traced back to plants. Every energy transfer results in some energy released as heat into the environment that cannot be used to do work, eventually most of the energy that goes into an entire ecosystem is released as heat into the environment as organisms in the ecosystem utilize energy in food to grow and function. Hence, as one moves up different levels in the ecosystem less and less energy is available to organisms. [much of this relates to 4 and 8] 


\section{Appendix D:}

Relationship Between NRC Framework and Hypothetical Learning Progression for the Flow of Matter \& Energy in Ecosystems 